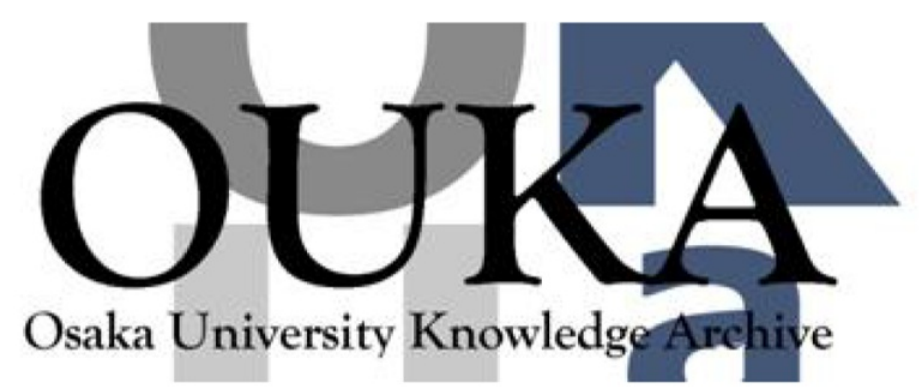

\begin{tabular}{|c|l|}
\hline Title & Structure of alternative and Jordan bimodules \\
\hline Author(s) & Jacobson, N. \\
\hline Citation & Osaka Mathematical Journal. 6(1) p. 1-p. 71 \\
\hline Issue Date & $1954-06$ \\
\hline oaire:version & VoR \\
\hline URL & https://doi.org/10.18910/8608 \\
\hline rights & \\
\hline Note & \\
\hline
\end{tabular}

Osaka University Knowledge Archive : OUKA

https://ir. Library. osaka-u. ac. jp/

Osaka University 
Osaka Mathematical Journal

Vo1. 6, No. 1, June, 1954

\title{
Structure of Alternative and Jordan Bimodules
}

\author{
by N. JACOBSON1)
}

The notion of a bimodule for a class of algebras defined by multilinear identities has been introduced by Eilenberg [13]. If $\mathfrak{A}$ is in the class of associative algebras or in the class of Lie algebras, then this notion is the familiar one for which we are in possession of well-worked theories. The study of bimodules (or representations) of Jordan algebras was initiated by the author in a recent paper [21]. Subsequently the alternative case was considered by Schafer [32]. In our paper we introduced the basic concepts of the Jordan theory and we proved complete reducibility of the bimodules and the analogue of Whitehead's first lemma for finite dimensional semi-simple Jordan algebras of characteristic 0 . Similar results on alternative algebras, based on those in the Jordan case, were obtained by Schafer. The principal tool in our paper was the notion of a Lie triple system. This permitted the application of important results on the structure and representation of Lie algebras to the problems on Jordan and alternative algebras. This method has one nice feature, namely, it is a general one which does not require a consideration of cases. On the other hand, it has the serious drawback that is unlikely that it can be adapted to the characteristic $p(\neq 0)$ case, since, as is well known, most of the important theorems on Lie algebras of characteristic 0 are false for Lie algebras of characteristic $p$.

Besides this question of the validity of the theorems for Jordan and alternative algebras of characteristic $p$ there is another important problem which was left open in the two papers cited, namely, the problem of determining the irreducible representations for finite dimensional semi-simple algebras. For this problem, too, the Lie method seems to be inappropriate.

In this paper we shall solve the two problems which we have indicated. An outline of the method which we shall use is as follows. In part I we develop certain general results on universal associative algebras and Peirce decompositions which permit a reduction of the

1) A major portion of this work was done while the author held a Guggenheim Memorial Fellowship. 
problems to various particular cases of these problems. Of importance are the special ways of constructing bimodules (e.g. special bimodules for Jordan algebras, associative bimodules for alternative algebras) and a criterion (Theorem 5.1) that a Jordan bimodule be obtainable by one of these constructions. In part II we consider the theory of alternative bimodules for matrix algebras and of Jordan modules for Jordan algebras of self-adjoint elements of matrix algebras. A noteworthy feature of this discussion is the intertwining of the Jordan and alternative theories. A key result in these considerations is a general structure theorem (Theorem 9.1) for Jordan algebras which permits the reduction of the theory of certain types of Jordan bimodules to alternative ones. While we confine ourselves to the application to representation theory here, it should be mentioned that this result can be used to effect substantial simplifications in the structure theory. We hope to indicate some of these applications in subsequent papers. Allso we should note that the methods developed in part II need not be confined to finite dimensional algebras. Thus these methods can also be applied to certain important types of algebras and rings which do not satisfy finiteness conditions (e.g. the Jordan algebra of self-adjoint transformations in Hilbert space)..2) In part III we study the representations of Jordan algebras which are defined by symmetric scalar products (or quadratic forms). The universal associative algebras for these Jordan algebras are the Clifford algebras and certain algebras which we shall call meson algebras, since they seem to have made their first appearance in the quantum theory of mesons. We determine the structure of these algebras. In part IV we specialize the theory to alternative and Jordan algebras of finite dimensions. The results of part II and III have to be combined and supplemented with the considerations of the exceptional simple alternative algebras (the Cayley algebras) and Jordan algebras $\left(M_{3}^{8}\right)$. The combination of these results yield the theorem on complete reducibility and the determination of the irreducible representations.

The next step which is indicated in the development of this type of representation theory is the consideration of the cohomology theory. While we do not as yet have satisfactory general definitions of the cohomology groups, we can apply the results of the present paper to obtain the analogues of the first two Whitehead lemmas for arbitrary characteristic. We hope to take up these questions, too, in subsequent papers.

2) Cf. Jacobson and Rickart [22] and [23]. 


\section{General Theory}

\section{Classes of algebras defined by identities}

We shall be concerned with algebras over a field $\Phi$. Nearly everything which we shall do can be done also for algebras over commutative rings, but for the sake of simplicity we shall confine our attention to the field case.

We define first the notion of a free non-associative algebra based on a vector space $\mathfrak{i}$ over $\Phi$. Consider the Kronecker product space $\mathfrak{B} \times \mathfrak{B}$ and the Kronecker product spaces $(\mathfrak{B} \times \mathfrak{B}) \times \mathfrak{B}$ and $\mathfrak{i} \times(\mathfrak{Z} \times \mathfrak{B})$. There is a natural isomorphism between the last two and one usually identifies these two spaces by means of this isomorphism. However, for the purposes of non-associative algebra one has to consider these two spaces as distinct objects and, in fact, as having no elements in common. Similarly, we can form five four-fold product spaces out of $\mathfrak{B}$ and in general we have $\frac{1}{n}\left(\begin{array}{c}2 n-2 \\ n-1\end{array}\right) n$-fold products of $\mathfrak{B}^{3}{ }^{3}$ These are obtained by Kronecker multiplication of any of the $r$-fold products by any of the $(n-r)$-fold products where $r=1,2, \cdots, n-1$. We now form the direct sum $\mathfrak{S}(\mathfrak{B})$ of all these $n$-fold products of $\mathfrak{B}, n=1,2, \ldots$ and we define the obvious multiplication in $\mathfrak{S}_{(\mathfrak{Z})}$. The result is called the free non-associative algebra based on the vector space $\mathfrak{B}$. Sometimes it is convenient to adjoin an identity element to $\mathfrak{S}(\mathfrak{B})$. This is done by forming the direct sum $\mathfrak{S}^{*}(\mathfrak{B})=\mathfrak{S}(\mathfrak{B}) \oplus(1)$ where $(1)$ is the one dimensional space of multiples of the identity 1 . We shall refer to $\mathfrak{S}^{*}(\mathfrak{B})$ as the free non-associative algebra with identity based on $\mathfrak{B}$.

Let $\mathfrak{A}$ be an arbitrary non-associative algebra over $\Phi$ and let $T$ be a linear mapping of $\mathfrak{B}$ into $\mathfrak{A}$. Then $T$ can be extended in one and only one way to a homomorphism of the free non-associative algebra $\mathfrak{S}(\mathfrak{B})$ into $\mathfrak{A}$.

Definition 1.1. Let $\{\boldsymbol{p}\}$ be a set of non-zero elements in the free non-associative algebra $\mathfrak{S}(\mathfrak{B})$. A non-associative algebra $\mathfrak{A}$ is said to satisfy the set of identities $\{p\}$ if every $p$ in this set is mapped into 0 by every homomorphism of $\mathfrak{S}(\mathfrak{B})$ into $\mathfrak{X}$.

Examples. (1) Let $e_{1}, e_{2}$ be linearly independent in $\mathfrak{B}$. Then $p=e_{1} \times e_{2}-e_{2} \times e_{1} \neq 0$ in $\subseteq(\mathfrak{B})$. Since the $e_{i}$ are linearly independent, there exists a linear transformation sending $e_{1}$ and $e_{2}$ into any two elements of an algebra $\mathfrak{A}$. Hence $\mathfrak{A}$ satisfies $p$ if and only if $a b=b a$

3) Cf. the author's Lectures in Abstract Algebra, Vol. I, p. 18. 
holds for all $a, b$ in $\mathfrak{A}$. (2) Let $e_{1}, e_{2}, e_{3}$ be linearly independent and take $p=\left(e_{1} \times e_{2}\right) \times e_{3}-e_{1} \times\left(e_{2} \times e_{3}\right)$. The algebras satisfying this identity are just the associative algebras. (3) Take $p=e_{1} \times e_{2}-e_{2} \times e_{1}$, $q=\left(e_{1} \times e_{2}\right) \times e_{3}-e_{1} \times\left(e_{2} \times e_{3}\right)$. The algebras satisfying these two identities are the commutative, associative algebras. (4) Let $p=e \times e$, $q=\left(e_{1} \times e_{2}\right) \times e_{3}+\left(e_{2} \times e_{3}\right) \times e_{1}+\left(e_{3} \times e_{1}\right) \times e_{2}$. These two identities define the class of Lie algebras. (5) Set $p_{1}=\left(e_{1} \times e_{1}\right) \times e_{2}-e_{1} \times\left(e_{1} \times e_{2}\right)$, $p_{2}=\left(e_{1} \times e_{2}\right) \times e_{1}-e_{1} \times\left(e_{2} \times e_{1}\right), p_{3}=\left(e_{2} \times e_{1}\right) \times e_{1}-e_{2} \times\left(e_{1} \times e_{1}\right)$. These identities define the alternative algebras. (6) The class of Jordan algebras is defined by the following two identities: $p=e_{1} \times e_{2}-e_{2} \times e_{1}$, $q=\left(\left(e_{1} \times e_{1}\right) \times e_{2}\right) \times e_{1}-\left(e_{1} \times e_{1}\right) \times\left(\epsilon_{2} \times e_{1}\right)$.

We shall not attempt a systematic investigation of identities of algebras. However, one or two remarks may be in order. First, we define a $T$-ideal in a free non-associative algebra $\mathfrak{S}(\mathfrak{B})$ as an ideal which is mapped into itself by every homomorphism of $\mathfrak{S}(\mathfrak{S})$ into itself. ${ }^{4)}$ Now let $\{\boldsymbol{p}\}$ be a set of non-zero elements in $\mathfrak{S}(\mathfrak{B})$ and let $\Omega$ be the $T$-ideal generated by this set. It is clear that $\Omega$ is the ideal generated by the images of the $p \in\{p\}$ under homomorphisms of $\mathfrak{S}(\mathfrak{B})$ into itself. It follows that if $\mathfrak{U}$ satisfies the identities in $\{\boldsymbol{p}\}$ then $\mathfrak{A}$ satisfies the identities in $\Re$.

If $\{p\}$ is an arbitrary set of non-zero elements in $\mathfrak{S}(\mathfrak{Z})$ and $\Re$ is the $T$-ideal generated by $\{p\}$ then $\mathfrak{S}(\mathfrak{Y}) / \Omega$ is called the free algebra based on $\mathfrak{Y}$ for the set of identities $\{p\}$. Since any homomorphism of $\mathfrak{S}(\mathfrak{B})$ into $\mathfrak{S}(\mathfrak{B}) / \mathscr{\Omega}$ is a product of a homomorphism of $\mathfrak{S}(\mathfrak{B})$ into itself by the natural homomorphism of $\mathfrak{S}(\mathfrak{B})$ into $\mathfrak{S}(\mathfrak{B}) / \Omega, \Omega$ is mapped into 0 by every homomorphism of $\mathfrak{S}(\mathfrak{B})$ into $\mathfrak{S}(\mathfrak{S}) / \Omega$. It follows that the free algebra $\mathfrak{S}(\mathfrak{S}) / \Omega$ satisfies the given set of identities $\{\boldsymbol{p}\}$. If $\mathfrak{A}$ is any algebra satisfying these identities, then any homomorphism of $\mathfrak{S}(\mathfrak{B})$ into $\mathfrak{A}$ maps $\Omega$ into 0 . Hence it induces a homomorphism of the free algebra $\mathfrak{S}(\mathfrak{B}) / \Re$ into $\mathfrak{A}$. It follows that any linear mapping of $\mathfrak{S}(\mathfrak{V}) / \mathfrak{R}$ into $\mathfrak{A}$ can be extended to a homomorphism of $\mathfrak{S}(\mathfrak{B}) / \mathscr{R}$ into $\mathfrak{U}$. If $\mathfrak{Y}$ is chosen large enough so that there exist homomorphisms of $\mathfrak{S}(\mathfrak{B})$ onto $\mathfrak{A}$ then $\mathfrak{A}=\mathfrak{S}(\mathfrak{V}) / \mathfrak{F}$ where $\mathfrak{\Im}$ is an ideal. Evidently $\mathfrak{\Im} \Re$ and $\mathfrak{U}$ is a homomorphic image of the free algebra $\mathfrak{S}(\mathfrak{B}) / \mathfrak{\Omega}$. Thus any algebra satisfying a set of identities is the homomorphic image of a suitable free algebra for these identities.

The free associative algebras will be particularly important in the sequel. We denote such an algebra by $\mathfrak{F}(\mathfrak{V})$ or, if the identity is

4) For associative algebras this notion has been introduced by Specht [33]. Cf. also Amitsur [6]. 
adjoined, by $\mathfrak{F}^{*}(\mathfrak{B})$. It is easy to see that $\mathfrak{F}(\mathfrak{B})$ can also be obtained in the following way: There is a natural isomorphism between any two $n$-fold Kronecker products of $\mathfrak{B}$ with itself. We shall now simply identify all of the products by means of these isomorphisms. The resulting space is denoted as $\mathfrak{B} \otimes \mathfrak{B} \otimes \cdots \otimes \mathfrak{B}$ and the product of $n$ vectors from $\mathfrak{B}$ by $v_{1} \otimes v_{2} \otimes \cdots \otimes v_{n}$. Then $\mathfrak{F}(\mathfrak{B})=\mathfrak{B} \oplus(\mathfrak{B} \otimes \mathfrak{V}) \oplus$ $(\mathfrak{S} \otimes \mathfrak{B} \otimes \mathfrak{B}) \oplus \cdots$ with the obvious multiplication.

We consider again the free non-associative algebra $\mathfrak{S}(\mathfrak{B})$. A subset of a $T$-ideal in $\mathfrak{S}(\mathfrak{B})$ is called a set of $T$-generators of the ideal if the smallest $T$-ideal containing these elements is the given $T$-ideal. Evidently, in considering identities, we can replace any set of $T$-generators by any other.

Let $e_{1}, e_{2}, \cdots, e_{n}$ be linearly independent elements of $\mathfrak{B}$. It is clear what is meant by a monomial (belonging to $\mathfrak{S}(\mathfrak{S})$ ) in $e_{1}, e_{2}, \cdots, e_{n}$, by the total degree of such a monomial and by its degree in a particular $e_{i}$. An element $p$ of $\mathfrak{S}(\mathfrak{B})$ is called multilinear in $e_{1}, e_{2}, \cdots e_{n}$ if it is a liner combination of monomials in $e_{1}, e_{2}, \cdots e_{n}$ all of which are of total degree $n$ and of degree 1 in each $e_{i}, i=1,2, \cdots, n$.

If $\mathfrak{B}$ has infinite dimensionality and if the characteristic of the base field is suitably restricted, then any $T$-ideal in $\mathfrak{S}(\mathfrak{B})$ has a set of multilinear $T$-generators. The proof of this is quite easy but we shall not give it. In the examples we listed above, the identities in (1)-(3) are multilinear. In (4) we replace $p$ by $e_{1} \times e_{2}+e_{2} \times e_{1}$. This gives an equivalent set of identities if the characteristic of $\Phi$ is $\neq 2$. In the alternative case (5), if the characteristic is $\neq 2$, we can use the multilinear identities:

$$
\begin{aligned}
& \left(e_{1} \times e_{2}\right) \times e_{3}-e_{1} \times\left(e_{2} \times e_{3}\right)-\left(e_{2} \times e_{3}\right) \times e_{1}+e_{2} \times\left(e_{3} \times e_{1}\right), \\
& \left(e_{1} \times e_{2}\right) \times e_{3}-e_{1} \times\left(e_{2} \times e_{3}\right)-\left(e_{3} \times e_{1}\right) \times e_{2}+e_{3} \times\left(e_{1} \times e_{2}\right), \\
& \left(e_{1} \times e_{2}\right) \times e_{3}-e_{1} \times\left(e_{2} \times e_{3}\right)+\left(e_{2} \times e_{1}\right) \times e_{3}-e_{2} \times\left(e_{1} \times e_{3}\right) .
\end{aligned}
$$

In the Jordan case (6) we have to assume that the characteristic is $\neq 2,3$. Then we can use the multilinear identities $e_{1} \times e_{2}-e_{2} \times e_{1}$ and

$$
\begin{aligned}
& \left(\left(e_{1} \times e_{2}\right) \times e_{3}\right) \times e_{4}+\left(\left(e_{1} \times e_{4}\right) \times e_{3}\right) \times e_{2}+e_{1} \times\left(\left(e_{2} \times e_{4}\right) \times e_{3}\right) \\
& -\left(e_{1} \times e_{2}\right) \times\left(e_{3} \times e_{4}\right)-\left(e_{1} \times e_{3}\right) \times\left(e_{2} \times e_{4}\right)-\left(e_{1} \times e_{4}\right) \times\left(e_{2} \times e_{3}\right) .
\end{aligned}
$$

These results can be proved by a simple polarization process. In the sequel we shall assume that the characteristic is $\neq 2$ for Lie and alternative algebras and $\neq 2,3$ for Jordan algebras. ${ }^{5)}$

5) As a matter of fact, the multilinear identity for Jordan algebras implies the original identity if the characteristic is $\neq 2$. We could therefore use it to define Jordan algebras of characteristic three. With this understanding, our results will be valid also for this characteristic. 


\section{Bimodules for non-associative algebras satisfying identities}

We consider now a fixed set $\{\boldsymbol{p}\}$ of multilinear identities and we let $\Gamma$ be the class of algebras satisfying these and $\mathfrak{A}$ a member of $\Gamma$. We propose to consider the simplest extension problem for $\mathfrak{A}$. Thus we take a vector space $\mathfrak{M}$ over $\Phi$ and we form the space $\mathfrak{F}=\mathfrak{U} \oplus \mathfrak{M}$. We retain the given multiplication in $\mathfrak{A}$, define $m_{1} m_{2}=0$ for $m_{i} \in \mathfrak{M}$ and we seek to define compositions $a m, m a$ for $a \in \mathfrak{A}, m \in \mathfrak{M}$ so that $(5$ will be an algebra in $\Gamma$ and $\mathfrak{M}$ will be an ideal in $\&$. Since $\mathfrak{M}$ is to be an ideal, we must have $a m, m a \in \mathfrak{M}$. Also the condition that \& be an algebra is equivalent to the bilinearity of the compositions $a m, m a$, that is, to the conditions

$$
\begin{aligned}
\left(\alpha_{1} a_{1}+\alpha_{2} a_{2}\right)\left(\mu_{1} m_{1}+\mu_{2} m_{2}\right)= & \alpha_{1} \mu_{1} a_{1} m_{1}+\alpha_{2} \mu_{1} a_{2} m_{1} \\
& +\alpha_{1} \mu_{2} a_{1} m_{2}+\alpha_{2} \mu_{2} a_{2} m_{2} \\
\left(\mu_{1} m_{1}+\mu_{2} m_{2}\right)\left(\alpha_{1} a_{1}+\alpha_{2} a_{2}\right)= & \mu_{1} \alpha_{1} m_{1} a_{1}+\mu_{1} \alpha_{2} m_{1} a_{2} \\
& +\mu_{2} \alpha_{1} m_{2} a_{1}+\mu_{2} \alpha_{2} m_{2} a_{2},
\end{aligned}
$$

$a_{1}, a_{2} \in \mathfrak{X} ; m_{1}, m_{2} \in \mathfrak{M} ; \alpha_{1}, \alpha_{2}, \mu_{1}, \mu_{2} \in \Phi$. We consider next the conditions that $\&$ satisfy the given multilinear identities. Since these identities are multilinear it suffices that they be satisfied for all choices of elements out of $\mathfrak{M}$ and out of $\mathfrak{A}$. If we choose all the arguments in $\mathfrak{A}$, then the identities do hold; for $\mathfrak{A} \in \Gamma$. Also, every product containing two elements of $\mathfrak{M}$ is 0 . Hence, necessary and sufflcient conditions that $\leftleftarrows \in \Gamma$ are that the results of substituting one $m \in \mathfrak{M}$ and the remaining elements in $\mathfrak{A}$ in the identities should be 0 . These substitutions must be done in every possible way, but of course, the resulting conditions may be redundant and reducible to a smaller set. These conditions define the notion of a bimodule for the algebra in the class $\Gamma$. The algebra $\mathbb{f}=\mathfrak{A} \oplus \mathfrak{M}$ constructed from the bimodule $\mathfrak{M}$ is called the split null extension determined by the given bimodule. It is clear that if $\mathfrak{A} \in \Gamma$ then $\mathfrak{A}$ itself can be regarded as a bimodule relative to the multiplication compositions $m a$, am defined in $\mathfrak{A}$. More generally, if $\mathfrak{A}$ is a subalgebra of an algebra $\mathfrak{B} \in \Gamma$ then we consider $\mathfrak{B}$ as an $\mathfrak{A}$-bimodule relative to the multiplication compositions $b a, a b, a \in \mathfrak{A}, b \in \mathfrak{B}$. The notions of sub-bimodules, difference bimodule, homomorphism, isomorphism, etc. are clear. If $\mathfrak{A}$ is an algebra that contains no ideals $\neq 0$ whose squares are 0 then the ideal $\mathfrak{M}$ in the split null extension $\mathbb{F}=\mathfrak{A} \oplus \mathfrak{M}$ contains every ideal $\mathfrak{R}$ of $\mathfrak{F}$ such that $\mathfrak{R}^{2}=0$. In this case it follows that the two bimodules $\mathfrak{M}_{1}$ and $\mathfrak{M}_{2}$ are isomorphic if there exists an isomorphism between the corresponding split null extensions $\mathfrak{F}_{1}=\mathfrak{A} \oplus \mathfrak{M}_{1}$ and 
$\mathfrak{F}_{2}=\mathfrak{A} \oplus \mathfrak{M}_{2}$ which is the identity on $\mathfrak{X}$. The converse holds without any restriction on $\mathfrak{A}$.

In the associative and Lie cases the concept of a bimodule specializes to familiar notations. Thus in the associative case, the conditions that $\&$ be associative are that

$$
\begin{gathered}
\left(a_{1} a_{2}\right) m=a_{1}\left(a_{2} m\right), \quad m\left(a_{1} a_{2}\right)=\left(m a_{1}\right) a_{2} \\
\left(a_{1} m\right) a_{2}=a_{1}\left(m a_{2}\right) .
\end{gathered}
$$

These, and (1), define the usual concept of an associative bimodule for A. It should be noted that this includes the usual notion of a right (left) module. For, if we have a right module with composition $m a$, $m \in M, a \in A$, then we obtain a bimodule by setting $a m \equiv 0$. In the Lie case we obtain the usual notion of a Lie bimodule, in which we have

$$
\begin{gathered}
a m=-m a \\
m\left(a_{1} a_{2}\right)=\left(m a_{1}\right) a_{2}-\left(m a_{2}\right) a_{1} .
\end{gathered}
$$

Since $a m=-m a$, we can drop one of these compositions and we may consider $\mathfrak{M}$ as just a right (or left) module relative to $\mathfrak{A}$.

The purpose of this paper is the study of alternative and Jordan bimodules. Hence we shall define these formally in the following two definitions.

Definition 2.1. Let $\mathfrak{A}$ be an alternative algebra. An alternative bimodule for $\mathfrak{A}$ is a vector space $\mathfrak{M}$ together with two compositions $a m, m a$ for a in $\mathfrak{A}, m$ in $\mathfrak{M}$ such that $a m, m a \in \mathfrak{M}$, the bilinearity condition (1) hold and

$$
\begin{aligned}
& A\left(a_{1}, m, a_{2}\right)=-A\left(m, a_{1}, a_{2}\right)=A\left(a_{2}, a_{1}, m\right) \\
& \quad=-A\left(a_{2}, m, a_{1}\right)
\end{aligned}
$$

where, in general,

$$
A(x, y, z) \equiv(x y) z-x(y z) .
$$

Definition 2.2. If $\mathfrak{I}$ is a Jordan algebra, a Jordan bimodule is a vector space $\mathfrak{M}$ with bilinear compositions $m a, a m, a \in \mathfrak{S}, m \in \mathfrak{M}$, $a m$ and $m a \in \mathfrak{M}$ such that

$$
\begin{gathered}
a m=m a \\
m a_{1} a_{2} a_{3}+m a_{3} a_{2} a_{1}+m\left(a_{1} a_{3} a_{2}\right) \\
=\left(m a_{1}\right)\left(a_{2} a_{3}\right)+\left(m a_{2}\right)\left(a_{1} a_{3}\right)+\left(m a_{3}\right)\left(a_{1} a_{2}\right) \\
=\left(m\left(a_{2} a_{3}\right)\right) a_{1}+\left(m\left(a_{1} a_{3}\right)\right) a_{2}+\left(m\left(a_{1} a_{2}\right)\right) a_{3}
\end{gathered}
$$


where, in general,

$$
x_{1} x_{2} \cdots x_{k} \equiv\left(\cdots\left(\left(x_{1} x_{2}\right) x_{3}\right) \cdots x_{k i}\right) .
$$

As in the Lie case, it is clear that a Jordan bimodule can also be considered as just a Jordan right or left module. Since $a m=m a$, nothing is lost in dropping one of the compositions. The condition for a right module is the one given above. For a left module we have a corresponding condition in which the $a$ 's appear to the left of Mr. In the sequel the term Jordan module will be used for right Jordan module.

\section{Relations to associative algebras}

It is clear from the definitions that associative algebras are alternative. Thus the notion of an alternative algebra is a generalization of that of an associative algebra. It is well known that Lie and Jordan algebras are also closely related to associative ones. For Lie algebras the relation is as follows. Let $\mathfrak{A}$ be an arbitrary associative algebra and define $[a b] \equiv a b-b a$ in $\mathfrak{A}$. If one replaces the associative multiplication $a b$ by the Lie multiplication [ab] one obtains a Lie algebra $\mathfrak{U}_{L}$. Moreover, it is well known ([8] and [36]) that every Lie algebra is isomorphic to a subalgebra of a suitable Lie algebra $\mathfrak{A}_{L}$. In a similar manner one obtains Jordan algebras from associative ones. Here we replace the associative composition $a b$ by the Jordan multiplication $\{a b\}=a b+b a$. The result is a Jordan algebra $\mathfrak{A}_{J}$. The situation is, however, somewhat different from the Lie case, since it is known that there exist Jordan algebras which are not subalgebras of any $\mathfrak{A}_{J}$. One must therefore distinguish two types of Jordan algebras: the special ones which are isomorphic to subalgebras of algebras of the form $\mathfrak{U}_{J}, \mathfrak{U}$ associative and the exceptional ones which are not. If $\mathfrak{A}$ is an alternative algebra, then it is well known (cf. [37]) that any two elements of $\mathfrak{A}$ generate an associative subalgebra. Since the defining Jordan identities are functions of pairs of elements, it is clear that if $\mathfrak{A}$ is alternative then the algebra $\mathfrak{H}_{J}$ obtained by substituting $\{a b\}=a b+b a$ for $a b$ in $\mathfrak{A}$ is a Jordan algebra. However, it is not difficult to see that these algebras are all special ([32] p. 3).

The relations which we have indicated have counterparts in the theory of bimodules for alternative and Jordan algebras. First, let $\mathfrak{U}$ be alternative, $\mathfrak{M}$ a vector space and $a m$ and $m a$ bilinear compositions defined for $a \in \mathfrak{A}, m \in \mathfrak{M}$ with values $a m, m a \in \mathfrak{M}$ such that $(2)$ holds. Then $\mathfrak{M}$ and the two compositions evidently define a bimodule for $\mathfrak{X}$. A bimodule of this type will be called an associative bimodule for the 
alternative algebra $\mathfrak{A}$. Next let $\mathfrak{\Im}$ be a Jordan algebra and let $\mathfrak{M}$ be a vector space, $m a$ a bilinear composition into $\mathfrak{M}$ such that

$$
m\left(a_{1} a_{2}\right)=\left(m a_{1}\right) a_{2}+\left(m a_{2}\right) a_{1}
$$

It is easy to verify that $\mathfrak{M}$ and $m a$ define a Jordan (right) module

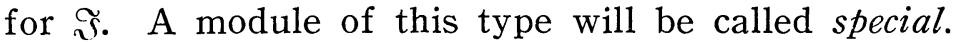

Now suppose that $\mathfrak{M}$ is both a special left module and a special right module for $\Im$. Thus we have compositions $m a$ and $a m$ satisfying (6) and the corresponding left condition $(a b) m=a(b m)+b(a m)$. We assume also that we have the associativity (or operator commutativity) condition: $(a m) b=a(m b)$. We now define a new bilinear composition

$$
\{m a\}=m a+a m
$$

in $\mathfrak{M}$. Then we can verify that $\mathfrak{M}$ and \{\} constitute a new Jordan module. We shall call this module the sum of the given two commmuting special modules.

In a certain sense one can form the sum of any two special modules for a Jordan algebra $\mathfrak{~}$. Then let $\mathfrak{M}_{1}$ and $\mathfrak{M}_{2}$ be two such modules and let $\mathfrak{M}=\mathfrak{M}_{1} \otimes \mathfrak{M}_{2}$. Then it is clear that we can define compositions $m a$ and $a m$ in $\mathfrak{M}$ by setting

$$
\begin{aligned}
\left(m_{1} \otimes m_{2}\right) a & =m_{1} \otimes m_{2} a \\
a\left(m_{1} \otimes m_{2}\right) & =m_{1} a \otimes m_{2} .
\end{aligned}
$$

These define $\mathfrak{M}$ as a left and a right Jordan module so that the associativity conditions hold. Hence

$$
\left\{\left(m_{1} \otimes m_{2}\right), a\right\}=m_{1} a \otimes m_{2}+m_{1} \otimes m_{2} a
$$

defines another Jordan module in $\mathfrak{M}$. We shall call this module the Kronecker sum of the two given special modules for $\mathfrak{\Im}$.

We again suppose that $\mathfrak{U}$ is alternative and that $\mathfrak{M}$ is an arbitrary bimodule for $\mathfrak{A}$. Then it is easy to deduce from the defining conditions (4) that

$$
\begin{aligned}
m(a b+b a) & =(m a) b+(m b) a \\
(a b+b a) m & =a(b m)+b(a m) .
\end{aligned}
$$

Evidently these relations mean that $\mathfrak{M}$ is a special left and a special right module for the Jordan algebra $\mathfrak{A}_{J}$. While these two special modules do not in general satisfy the associativity condition, it is nevertheless true that the composition $\{m a\}=m a+a m$ defines another Jordan module for $\mathfrak{A}_{J}$ (cf. [32] p. 3). 


\section{Birepresentations. Universal associative algebras}

Up to this point we have emphasized the module point of view in the representation theory. It is fundamental also to consider the strictly representation approach in which the linear mappings $R_{a}: m \rightarrow m a$ and $L_{a}: m \rightarrow a m$ play the predominant role. The concept of bimodule for an algebra $\mathfrak{A}$ in a class of algebras defined by multilinear identities is equivalent to that of birepresentation for $\mathfrak{U}$ in $\Gamma$. We describe this to consist of a pair of linear mappings $a \rightarrow R_{a}$, $a \rightarrow L_{a}$ of $\mathfrak{A}$ into the space of linear transformations of a vector space $\mathfrak{M}$ where the $L_{a}$ and $R_{a}$ satisfy relations which are imposed on the mappings $m \rightarrow a m, m \rightarrow m a$ in a bimodule for $\mathfrak{A}$. We shall not attempt to make this explicit in the general case but shall confine our attention to the two cases of intesest here. These are given in the following definitions.

Definition 4.1. If $\mathfrak{A}$ is an alternative algebra we define an (alternative) birepresentation $(L, R)$ for $\mathfrak{A}$ by linear transformations in the vector space $\mathfrak{M}$ as two linear mappings $L: a \rightarrow L_{a},\left(R: a \rightarrow R_{a}\right.$ of $\mathfrak{A}$ into the space of linear transformations of $\mathfrak{M}$ into itself such that

$$
\begin{aligned}
{\left[L_{a_{1}} R_{a_{2}}\right] } & =R_{a_{1} a_{2}}-R_{a_{1}} R_{a_{2}}=L_{a_{2} a_{1}}-L_{a_{1}} L_{a_{2}} \\
& =\left[R_{a_{1}} L_{a_{2}}\right]
\end{aligned}
$$

where, as usual, $[X Y] \equiv X Y-Y X)$.

Definition 4.2. If $\Im$ is a Jordan algebra, we define a (Jordan) birepresentation $(L, R)$ for $\mathfrak{\Im}$ by linear transformations in a vector space $\mathfrak{M}$ as two linear mappings $L: a \rightarrow L_{a}, R: a \rightarrow R_{a}$ of $\Im$ into the space of linear transformations' in $\mathfrak{M}$ such that

$$
\begin{aligned}
& L_{a}=R_{a} \\
R_{a_{1}} R_{a_{2}} R_{a_{3}} & +R_{a_{3}} R_{a_{2}} R_{a_{1}}+R_{a_{1} a_{3} a_{2}}=R_{a_{1}} R_{a_{2} a_{3}} \\
& +R_{a_{2}} R_{a_{1} a_{3}}+R_{a_{3}} R_{a_{1} a_{2}}=R_{a_{2} a_{3}} R_{a_{1}}+R_{a_{1} a_{3}} R_{a_{2}} \\
& +R_{a_{1} a_{2}} R_{a_{3}} .
\end{aligned}
$$

If we drop the $L_{a}$ we have a representation for $\Im$. The concept of bimodule and birepresentation are equivalent in the following sense: If $\mathfrak{M}$ is a bimodule for $\mathfrak{A}$ or $\mathfrak{\Im}$ then the linear mappings $L_{a}: m \rightarrow a m$ and $R_{a}: m \rightarrow m a$ define a birepresentation. Conversely, if a birepresentation acting in $\mathfrak{M}$ is given, then the composition $m a \equiv m R_{a}$ and $a m \equiv m L_{a}$ define $\mathfrak{M}$ as a bimodule. In a similar 
fashion, the notion of an associative bimodule for an alternative algebra $\mathfrak{A}$ is equivalent to an associative birepresentation $(L, R)$ defined by the conditions

$$
R_{a_{1} a_{2}}=R_{a_{1}} R_{a_{2}},\left[L_{a_{1}} R_{a_{2}}\right]=0, \quad L_{a_{1} a_{2}}=L_{a_{2}} L_{a_{1}} .
$$

We remark that if we know that $(L, R)$ is a birepresentation for the alternative algebra $\mathfrak{A}$, then any one of the conditions (11) is sufficient that it be associative. If $\mathfrak{\Im}$ is a Jordan algebra then a special (bi) representation $S$ for $\Im$ is a linear mapping $a \rightarrow S_{a}$ such that

$$
S_{a_{1} a_{2}}=S_{a_{1}} S_{a_{2}}+S_{a_{2}} S_{a_{1}} .
$$

If $S^{(1)}$ and $S^{(2)}$ are two such representations acting in the same space, the associativity condition for modules corresponds to the requirement that $\left[S_{a}^{(1)} S_{b}^{(2)}\right]=0$ for all $a, b$. In this case the mapping $R_{a}=S_{a}^{(1)}+S_{a}^{(2)}$ define a representation (in the general sense) which we call the sum of the two commuting special representations.

In considering a set of linear transformations it is often useful to pass to the enveloping associative algebra, that is, the (associative) algebra generated by the set. In the case of a birepresentation, the study of this enveloping algebra of the $L_{a}$ and the $R_{a}$ is facilitated by the introduction of the universal associative algebra for the birepresentations. Suppose first that $\mathfrak{A}$ is alternative. Let $\mathfrak{i}=\mathfrak{A} \oplus \mathfrak{R}^{\prime}$ where $\mathfrak{A}^{\prime}$ is a vector space isomorphic to $\mathfrak{A}$ under a correspondence $a \rightarrow a^{\prime}$. We now form the free associative algebra $\mathfrak{F}(\mathfrak{B})$ and the difference algebra $\mathfrak{U}(\mathfrak{A})=\mathfrak{F}(\mathfrak{B}) / \mathfrak{R}$ where $\Re$ is the ideal in $\mathfrak{F}$ generated by the elements of the following types:

$$
\begin{aligned}
& a_{1}^{\prime} \otimes a_{2}-a_{2} \otimes a_{1}{ }^{\prime}-a_{1} a_{2}+a_{1} \otimes a_{2} \\
& a_{1} a_{2}-a_{1} \otimes a_{2}-\left(a_{2} a_{1}\right)^{\prime}+a_{1}{ }^{\prime} \otimes a_{2}{ }^{\prime} \\
& \left(a_{2} a_{1}\right)^{\prime}-a_{1}{ }^{\prime} \otimes a_{2}{ }^{\prime}-a_{1} \otimes a_{2}{ }^{\prime}+a_{2}{ }^{\prime} \otimes a_{1}, \quad a_{i} \in \mathfrak{A} .
\end{aligned}
$$

Let $\bar{a}$ denote the coset of $a \in \mathfrak{A}$ in $\mathfrak{u}(\mathfrak{U})$ and $\bar{a}^{\prime}$ that of $a^{\prime} \in \mathfrak{Y} \mathfrak{X}^{\prime}$ and denote multiplication in $\mathfrak{u}(\mathfrak{H})$ in the usual way (by $\otimes)$. Then we have the relations (9) for $\bar{a}^{\prime}, \bar{a}$ (in place of $L_{a}, R_{a}$ ). It follows that if $(L, R)$ is a birepresentation of $\mathfrak{A}$ then $\bar{a}^{\prime} \rightarrow L_{a}, \bar{a} \rightarrow R_{a}$ define a representation (in the usual sense) of the associative algebra $\mathfrak{U}(\mathfrak{U})$. Thus we can consider $\mathfrak{M}$ as a right (associative) $\mathfrak{U}(\mathfrak{H})$-module. Conversely, any right $\mathfrak{U}(\mathfrak{A})$-module defines an alternative $\mathfrak{A}$-bimodule. For these reasons we shall call $\mathfrak{u}(\mathfrak{A})$ the universal associative algebra for the birepresentations (bimodules) of $\mathfrak{A}$. Every alternative algebra has a birepresentation which is $1-1$ in the sense that $a \rightarrow L_{a}$ and $a \rightarrow R_{a}$ 
are $1-1$. Thus, if $\mathfrak{A}$ has an identity then the regular birepresentation $(\mathfrak{M}=\mathfrak{U})$ has this property and if $\mathfrak{U}$ does not have an identity then the regular birepresentation of $\mathfrak{U}$ acting in the algebra $\mathfrak{U}+\Phi 1$ obtained by adjoining an identity has the property. (One verifies that $\mathfrak{Y}+\Phi 1$ is alternative.) It now follows that the mapping $a \rightarrow \bar{a}$ of $\mathfrak{A}$ into $\mathfrak{U}(\mathfrak{U})$ is $1-1$. We may therefore identify $\mathfrak{U}$ with this image in $\mathfrak{u}(\mathfrak{A})$. Similarly, $a^{\prime} \rightarrow \bar{a}^{\prime}$ is $1-1$ and we may identify $\mathfrak{A}^{\prime}$ with its image in $\mathfrak{U}(\mathfrak{A})$. It is also convenient to consider $\mathfrak{U}^{\prime}$ as an (alternative) algebra which is anti-isomorphic to $\mathfrak{A}$ by means of the correspondence $a \rightarrow a^{\prime}$. Thus we introduce the multiplication $a^{\prime} b^{\prime}=(b a)^{\prime}$. In the sequel we shall make these identifications and introduce the multiplication indicated in $\mathfrak{U}^{\prime}$. The alternative multiplication in $\mathfrak{A}$ and $\mathfrak{U}^{\prime}$ will be distinguished from the associative multiplication in $\mathfrak{U}(\mathfrak{A})$ by the use of the notation $\otimes$ for the latter.

If $\Im$ is a Jordan algebra, we form the free associative algebra $\mathfrak{\Im}(\Im)$ for the vector space $\Im$ and we work modulo the ideal $\Re$ generated by the elements:

$$
\begin{aligned}
& a_{1} \otimes a_{2} \otimes a_{3}+a_{3} \otimes a_{2} \otimes a_{1}+a_{1} a_{3} a_{2}-a_{1} \otimes a_{2} a_{3}-a_{2} \otimes a_{1} a_{3}-a_{3} \otimes a_{1} a_{2}, \\
& a_{1} \otimes a_{2} a_{3}+a_{2} \otimes a_{1} a_{3}+a_{3} \otimes a_{1} a_{2}-a_{2} a_{3} \otimes a_{1}-a_{1} a_{3} \otimes a_{2}-a_{1} a_{2} \otimes a_{3} .
\end{aligned}
$$

The algebra $\mathfrak{u}(\mathfrak{\Im}) \equiv \mathfrak{F}(\mathfrak{\Im}) / \Re$ is called the universal associative algebra for the representations (modules) of $\Im$. As in the alternative case, any representation of $\mathfrak{\Im}$ determines one of the associative algebra $\mathfrak{u}(\mathfrak{\Im})$ and conversely. Also since there exist $1-1$ representations of $\mathfrak{\Im}$, we can identify $\mathfrak{\Im}$ with a subset of $\mathfrak{u}(\mathfrak{\Im})$.

The introduction of the universal associative algebras for the birepresentations enables one to split the representation problem into two parts: (1) determination of the structure of $\mathfrak{U},(2)$ representation theory for the associative algebra $\mathfrak{u}$. In practice, however, it seems to be difficult to treat (1) as a separate problem. Only in some special cases is it feasible to attack this directly.

It is useful at times to consider also the enveloping Lie algebra of the $L_{a}$ and $R_{a}$ furnished by a birepresentation. For this purpose one needs the Lie relations connecting the $R$ 's and the $L$ 's. In the alternative case these are

$$
\begin{aligned}
{\left[\left[L_{a} L_{c}\right] L_{b}\right] } & =L_{2 A(a, b, c)+\left[\left\{a_{e}\right] b\right]} \\
{\left[\left[R_{a} R_{c}\right] R_{b}\right] } & =R_{2 A(a, b, c)+[\{a \cdot] b]} \\
{\left[L_{a} R_{b}\right] } & =\frac{1}{2} R_{\{a b]}-\frac{1}{2}\left[R_{a} R_{b}\right] \\
& =-\frac{1}{2} L_{[a b]}-\frac{1}{2}\left[L_{a} L_{b}\right] .
\end{aligned}
$$


In the Jordan case, we have

$$
\left[\left[R_{a} R_{b}\right] R_{c}\right]=R_{A(b, c, a)} .
$$

One can also introduce universal Lie algebras for these relations, but we shall not require these in the sequel. ${ }^{6}$ )

\section{Properties of the universal associative algebras}

Let $\mathfrak{\Im}$ be a Jordan algebra and $\mathfrak{u}(\mathfrak{\Im}) \supset \mathfrak{\Im}$ the universal associative algebra. We note first that $\mathfrak{u}(\mathfrak{\Im})$ has an involution which is characterized by the property that the elements of $\Im$ are self-adjoint. It is well known and trivial that the free associative algebra $\mathfrak{F}(\mathfrak{\Im})$ has an involution such that the elements of the generating subspace $\mathfrak{\Im}$ are self-adjoint. This involution will induce an involution in $\mathfrak{U}(\mathfrak{\Im})=\mathfrak{F}(\mathfrak{\Im}) / \mathfrak{\Omega}$ provided that it maps $\Omega$ into itself. To see that this is the case we observe that the generators of the second type listed in (14) are skew relatise to the involution in $\mathfrak{F}$ while a generator of the first type is mapped into $a_{1} \otimes a_{2} \otimes a_{3}+a_{3} \otimes a_{2} \otimes a_{1}+a_{1} a_{3} a_{2}-a_{2} a_{3} \otimes a_{1}-a_{1} a_{3} \otimes a_{2}-a_{1} a_{2} \otimes a_{3}$ and this is expressible in terms of the given generators. Since $\mathfrak{u}(\mathfrak{\Im})$ is generated by $\Im$, it is clear that the involution in $\mathfrak{u}(\mathfrak{\Im})$ is the only one which leaves invariant the elements of $\Im$. We shall call this involution the fundamental involution in $\mathfrak{u}(\mathfrak{\Im})$.

We shall introduce next several other universal associative algebras for a Jordan algebra and shall consider their relation to $\mathfrak{U}(\mathfrak{\Im})$. The first of these is the universal associative algebra for the special representations. This is defined to be the algebra $\mathfrak{U}_{s}(\mathfrak{\Im})=\mathfrak{F}(\mathfrak{\Im}) / \Re_{s}$ where $\Re_{s}$ is the ideal in $\mathfrak{F}(\mathfrak{\Im})$ generated by the elements of the form $a \otimes b+b \otimes a-a b$. We denote the coset $a+\Re_{s}$ of $a$ in $\mathfrak{u}_{s}(\mathfrak{I})$ by $a_{s}$. Then it is clear that if $a \rightarrow S_{a}$ is a special representation of $\mathfrak{\Im}, a_{s} \rightarrow S_{a}$ determines a representation of the associative algebra $\mathfrak{u}_{s}$. More generally, if $a \rightarrow \bar{a}$ is a homomorphism of $\mathfrak{\Im}$ into any special Jordan algebra $\mathfrak{B}_{J}$, then $a_{s} \rightarrow \bar{a}$ can be extended to a homomorphism of $\mathfrak{U}_{s}$ into $\mathfrak{B}$. The algebra $\mathfrak{u}_{s}$ has been used before for the study of homomorphisms of Jordan algebras into special ones ([9] and [15]). Evidently the mapping $a \rightarrow a_{s}$ is $1-1$ if and only if $\mathfrak{\Im}$ is a special Jordan algebra. Since every special representation is a representation of $\mathfrak{\Im}$ and since $\mathfrak{U}_{s}$ has a 1-1 representation, $a \rightarrow a_{s}$ can be extended to a homomorphism of $\mathfrak{U}(\mathfrak{\Im})$ onto $\mathfrak{u}_{s}(\mathfrak{\Im})$. The algebra $\mathfrak{u}_{s}$ has an involution which leaves the elements $a_{s}$ fixed. This follows from the observation that the gener-

6) For the Jordan case this has been done in Jacobson [21]. 
ators $a \otimes b+b \otimes a-a b$ of the ideal $\Re_{s}$ are self-adjoint. Since the involution in $\mathfrak{U}_{s}$ is characterized by its property of leaving the $a_{\varepsilon}$ invariant, we shall call it the fundamental involution in $\mathfrak{u}_{s}(\mathfrak{\Im})$.

Now suppose that $\Im$ is a Jordan algebra with an identity element 1. A module $\mathfrak{M}$ for $\mathfrak{\Im}$ will be called unital if $m 1=m$ for all $m \in \mathfrak{M}$. In considering these modules one introduces the universal associative algebra for the unital representions. This is the algebra $\mathfrak{U}_{1}(\mathfrak{\Im})=\mathfrak{F}(\mathfrak{\Im}) / \Re_{1}$ where $\Re_{1}$ is the ideal generated by the $\Re$ used to define $\mathfrak{U}(\mathfrak{\Im})$ and the elements $a \otimes 1-a, 1 \otimes a-a, a \in \mathfrak{\Im}$. The problem of determining the structure of $\mathfrak{u}(\mathfrak{\Im})$ can be reduced to this problem for $\mathfrak{u}_{s}(\mathfrak{\Im})$ and $\mathfrak{u}_{1}(\mathfrak{\Im})$; for it is known that $\mathfrak{u}(\mathfrak{\Im}) \cong \mathfrak{U}_{\boldsymbol{s}}(\mathfrak{\Im}) \oplus \mathfrak{U}_{1}(\mathfrak{\Im})$ (in the algebra sense). ${ }^{7}$ In $\mathfrak{U}_{s}(\mathfrak{\Im})$ we have the relations $2 \cdot 1_{s}^{2}=1_{s}$ and $1_{s} a_{s}+a_{s} 1_{s}=a_{s}$. From these it follows that $u_{n}=2 \cdot 1_{s}$ is the identity element of $\mathfrak{u}_{s}(\mathfrak{F})$. Hence in considering special representations it is natural to assume that $S_{1}=\frac{1}{2} 1$. It is clear that the sum of two commuting special modules of this type is unital.

Now form the Kronecker product algebra $\mathfrak{u}_{s} \otimes \mathfrak{U}_{s}$ and let $\mathfrak{u}_{s}^{(2)}(\mathfrak{\Im})$ denote the subalgebra of $\mathfrak{u}_{s} \otimes \mathfrak{u}_{s}$ generated by the elements $a_{2}=a_{s} \otimes u$ $+u \otimes a_{s}$. Let $S^{(1)}$ and $S^{(2)}$ be two commuting special representations with $S_{1}^{(i)}=\frac{1}{2}$. Then the mappings $a_{s} \otimes u \rightarrow S_{a}^{(1)}, u \otimes a_{s} \rightarrow S_{a}^{(2)}$ homomorphisms mapping the identity element $1_{2}$ of $\mathfrak{u}_{s} \otimes \mathfrak{u}_{s}$ onto the identity transformation. It follows that these homomorphisms can be extended to a homomorphism of $\mathfrak{u}_{s} \otimes \mathfrak{U}_{s}$ onto the algebra generated by the $S_{a}^{(1)}$ and the $S_{a}^{(2)}$. Hence we have a homomorphism of $\mathfrak{u}_{s}^{(2)}(\mathfrak{\Im})$ mapping $a_{2}=a_{s} \otimes u+u \otimes a_{s}$ onto $R_{a} \equiv S_{a}^{(1)}+S_{a}^{(2)}$. For this reason we shall refer to $\mathfrak{H}_{s}^{(2)}(\mathfrak{\Im})$ as the universal associative algebra of sums of commuting special representations. Since $1_{2}$ is the identity element of $\mathfrak{U}_{s}^{(2)}(\mathfrak{\Im})$ it follows easily that there is a homomorphism of $\mathfrak{u}_{1}(\mathfrak{\Im})$ onto $\mathfrak{U}_{s}^{(2)}(\Im)$ mapping $a_{1}$ onto $a_{2}$. We can now summarize the relations among the various universal associative algebras for a Jordan algebra with an identity in the following diagrams:

$$
\mathfrak{u}=\mathfrak{u}_{1} \oplus \mathfrak{u}_{s}, \quad \mathfrak{u}_{1} \rightarrow \mathfrak{u}_{s}^{(2)} \rightarrow 0 .
$$

In a number of important cases the homomorphism of $\mathfrak{U}_{1}$ onto $\mathfrak{U}_{s}^{(2)}$ is an isomorphism. In these cases the problem of determining the structure of $\mathfrak{U}$ is . largely reduced to that of $\mathfrak{u}_{s}$. An important tool for establishing the isomorphism of $\mathfrak{u}_{1}$ and $\mathfrak{u}_{s}^{(2)}$ is the following general criterion.

7) Jacobson $[21]$, p. 517 . 
Theorem 5.1. Let $\mathfrak{I}$ be a special Jordan algebra, $\mathfrak{M}$ a module for $\mathfrak{\Im}$ and $\mathfrak{F}=\mathfrak{\Im} \oplus \mathfrak{M}$ the corresponding split null extension. Then $\mathfrak{M}$ is isomorphic to a submodule of a sum of two commuting special modules if and only if (5) is special.

Proof. Assume first that $\mathfrak{M}$ is ismorphic to a submodule of a sum $\mathfrak{R}$ of two commuting special modules. Then $\mathfrak{F}=\mathfrak{F} \oplus \mathfrak{M}$ is isomorphic to a subalgebra of the extension $\Im \oplus \mathfrak{N}$. Hence we may assume that $\mathfrak{M}$ is a sum of two commuting special modules. Thus we have two commuting special compositions $a m, m a$ in $\mathfrak{M}$ and the given composition is $\{a m\}=a m+m a$. Let $\mathfrak{H}_{s}$ be the universal associative algebra of the special representations of $\mathfrak{\Im}$ and let $a_{s}$ denote the element in this algebra corresponding to $a \in \Im$. If we set $a_{s} m=a m, m a_{s}=m a$, then we can consider $\mathfrak{M}$ as a biomodule for $\mathfrak{U}_{s}$. This is clear since $\left(a_{s} m\right) b_{s}=a_{s}\left(m b_{s}\right)$. We can therefore construct the corresponding associative null extension $\mathfrak{u}_{s} \oplus \mathfrak{M}$. Since $\mathfrak{\Im}$ is special, $a \rightarrow a_{s}$ is $1-1$; hence $a+m \rightarrow a_{s}+m$ is $1-1$ of $\mathbb{E}$ into $\mathfrak{H}_{s} \oplus \mathfrak{M}$. Since

$$
\left(a_{s}+m\right)\left(b_{s}+m^{\prime}\right)+\left(b_{s}+m^{\prime}\right)\left(a_{s}+m\right)=(a b)_{s}+\left\{m^{\prime} a\right\}+\{m b\},
$$

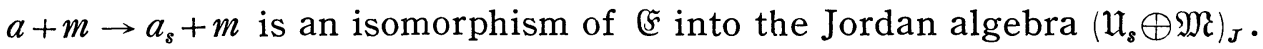
Hence $\mathbb{F}$ is special. Conversely, assume that $\mathbb{F}=\mathfrak{\Im} \oplus \mathfrak{M}$ is special. Then we have an isomorphism $\theta$ of $\mathfrak{F}$ into $\mathfrak{A}_{J}, \mathfrak{A}$ associative. Now we can consider $\mathfrak{A}$ as a special $\mathfrak{\Im}$-module in two ways by setting $v a=v a^{\theta}, a v=a^{\theta} v, a \in \mathfrak{F}, v \in \mathfrak{A}$. These two modules commute so that we can form their sum in which the composition is $\{v a\}=v a+a v$. Evidently $\mathfrak{M}^{\theta}$ is a submodule of this module. Since $\left\{m^{\theta} a\right\}=\left(m^{\theta} a^{\theta}\right.$ $\left.+a^{\theta} m^{\theta}\right)=(m a)^{\theta}, \theta$ is a module isomorphism of $\mathfrak{M}$ onto $\mathfrak{M}^{\theta}$. Hence $\mathfrak{M}$ is isomorphic to a submodule of a sum of two commuting special modules.

The theory of the universal associative algebra for the birepresentations of an alternative algebra $\mathfrak{A}$ does not appear to be as interesting or as useful as that of a Jordan algebra. We note only that $\mathfrak{U}(\mathfrak{A})$ has an involution which exchanges the element $a \in \mathfrak{A}$ and its image $a^{\prime} \in \mathfrak{X}^{\prime}$. We recall finally a fundamental result which is common to the two theories: The universal associative algebra of the birepresentations of an alternative or a Jordan algebra of finite dimensions is finite dimensional ([21] p. 519 and [32] p. 10).

\section{Peirce decompositions}

Let $\mathfrak{A}$ be alternative and $a \rightarrow R_{a}, a \rightarrow L_{a}$ a birepresentation of $\mathfrak{A}$ 
acting in $\mathfrak{M}$. The defining conditions give $\left[R_{a} L_{a}\right]=0$. Hence if $e^{2}=e$ then, by $(9), R_{e}^{2}=R_{e}, L_{e}^{2}=L_{e}$. These relations imply that we have a Peirce decomposition of $\mathfrak{M}$ relative to $e$, that is,

$$
\mathfrak{M}=\mathfrak{M}_{11} \oplus \mathfrak{M}_{10} \oplus \mathfrak{M R}_{01} \oplus \mathfrak{M l}_{00}
$$

where

$$
\mathfrak{M}_{i j}=\left\{m_{i j} \mid m_{i j} e=\delta_{j_{1}} m_{1 j}, e m_{i j}=\delta_{i 1} m_{i j}\right\} .
$$

Assume next that $\mathfrak{A}$ has an identity element 1 . Then we have the Peirce decomposition (18) for $e=1$. Moreover, $\left\{R_{1} R_{a}\right\}=2 R_{a}$. This, and $R_{1}^{2}=R_{1}$ imply that $R_{1} R_{a}=R_{a}=R_{a} R_{1}$. Similarly, $L_{1} L_{a}=L_{a}=L_{a} L_{1}$. It follows that $\left[R_{1} L_{a}\right]=0=\left[L_{1} R_{a}\right]$ and these relations imply that the $\mathfrak{M}_{i j}$ in the Peirce decomposition are sub-bimodules of $\mathfrak{M}$. In the sub-bimodule $\mathfrak{M}_{00}$, we have $R_{1}=0=L_{1}$. This implies that $R_{a}=0=L_{a}$ for all $a$ in $\mathfrak{A}$. Hence $\mathfrak{M}_{00}$ is a trivial bimodule in which the birepresentation is 0 . In the submodule $\mathfrak{M}_{01}$ we have $L_{1}=0, R_{1}=1$. Hence $L_{a}=0$ and $R_{a} R_{b}=R_{a b}$. Thus this bimodule is associative; similarly the bimodule $\mathfrak{M}_{10}$ is associative.

Assume now that $\mathfrak{M}$ is unital and let $\left\{e_{i} \mid i=1,2, \cdots, r\right\}$ be a set of orthogonal idempotent elements in $\mathfrak{A}$ such that $\sum e_{i}=1$. The argument used before shows that if $e$ is idempotent and $a$ satisfies $e a=a=a e$, then $R_{a} R_{e}=R_{a}=R_{e} R_{a}, L_{a} L_{e}=L_{a}=L_{e} L_{a}$ and $\left[R_{e} L_{a}\right]=0$ $=\left[L_{e} R_{a}\right]$. If $e a=0=a e$, then $f a=a=a f$ for $f=1-e$. It follows that $R_{a} R_{e}=0=R_{e} R_{a}, L_{a} L_{e}=0=L_{e} L_{a},\left[R_{e} L_{a}\right]=0=\left[L_{e} R_{a}\right]$. In particular, we see that the $R_{e_{i}}\left(L_{e_{i}}\right)$ are orthogonal idempotent elements whose sum is 1 and that the $R_{e_{i}}$ commute with the $L_{e_{j}}$. It follows that we have the Peirce decomposition

$$
\mathfrak{M}=\sum_{i, j=1}^{r} \oplus \mathfrak{M}_{i j}
$$

where $\Sigma \oplus$ denotes direct sum and

$$
\mathfrak{M}_{i j}=\left\{m_{i j} \mid e_{r} m_{i j}=\delta_{i r} m_{i j}, m_{i j} e_{r}=\delta_{j r} m_{i j}\right\} \text {. }
$$

Next let $\mathfrak{A}=\mathfrak{A}_{1} \oplus \mathfrak{A}_{2} \oplus \cdots \oplus \mathfrak{A}_{r}$ where the $\mathfrak{A}_{\boldsymbol{i}}$ are ideals. Then $1=\sum u_{i}, u_{i} \in \mathfrak{R}_{i}$ and the $u_{i}$ are orthogonal idempotent elements. Let $\mathfrak{M}=\mathfrak{M}_{01}$ be a bimodule in which $L_{1}=0, R_{1}=1$. Then it is easy to see that $\mathfrak{M}=\sum \oplus \mathfrak{M}_{i}, \mathfrak{M}_{i}=\mathfrak{M} R_{u_{i}}$ and this is a sub-bimodule which is annihilated by all the $\mathfrak{A}_{\mathfrak{j}} \neq \mathfrak{A}_{i}$. A similar remark can be made for bimodules of the type $\mathfrak{M}=\mathfrak{M}_{10}$. Next suppose that $\mathfrak{M}$ is unital. Suppose $(20)$ is the Peirce decomposition of $\mathfrak{M}$ relative to these idempotents. If $a_{i} \in \mathfrak{N}_{i}, a_{i} u_{i}=a_{i}=u_{i} a_{i}$ and $a_{i} u_{j}=0=u_{j} a_{i}$ for $j \neq i$. It 
follows that $R_{a_{i}}$ and $L_{a_{i}}$ commute with all the $R_{u_{k}}, L_{u_{k}}$. Hence, the $R_{u_{i}}$ and $L_{u_{i}}$ commute with all the $R_{a}$ and $L_{a}$. It follows that the $\mathfrak{M}_{i j}$ are sub-bimodules of the unital bimodule $\mathfrak{M}$. As before, if $i \neq j$, then $\mathfrak{M}_{i j}$ is associative. Moreover, $a_{i} \rightarrow L_{a_{i}}$ is an antihomomorphism of $\mathfrak{A}_{i}$ into the algebra of linear transformations in $\mathfrak{M}_{i j}$ and $a_{j} \rightarrow R_{a j}$ is a homomorphism of $\mathfrak{A}_{j}$ into this algebra. Also, $\mathfrak{M}_{i j} \mathfrak{A}_{k}=0=\mathfrak{A}_{k} \mathfrak{M}_{i k}$ for $k \neq i, j$. It remains to consider the $\mathfrak{M}_{i i}$. One sees that these are untial $\mathfrak{A}_{i}$-bimodules which are annihilated left and right by the $\mathfrak{A}_{\jmath}, j \neq i$.

Similar results hold for Jordan algebras. Thus let $e$ be an idempotent element in a Jordan algebra $\Im$ and let $a \rightarrow R_{a}$ be a representation of $\Im$ in $\mathfrak{M}$. Then $R_{e}\left(R_{e}-1\right)\left(2 R_{e}-1\right)=0$ so that we have the Peirce decomposition

$$
\mathfrak{M}=\mathfrak{M}_{1} \oplus \mathfrak{M}_{\frac{1}{2}} \oplus \mathfrak{M}_{0}
$$

where

$$
\mathfrak{M}_{i}=\left\{m_{i} \mid m_{i} R_{e}=i m_{i}\right\} .
$$

If $e=1$ is the identity element in $\Im$ then $\left[R_{1} R_{a}\right]=0$ for all $a$. Hence the $\mathfrak{M}_{i}$ in the Peirce decomposition for 1 are submodules. Since, for $m \in \mathfrak{M}$,

$$
\begin{aligned}
m 1 a b+m b a 1+m(a b) & =(m 1)(a b)+m a b+m b a, \\
m(a b) & =m a b+m b a
\end{aligned}
$$

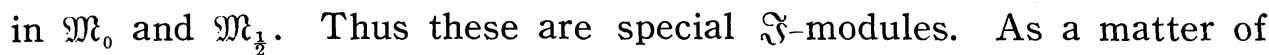
fact, it is clear that $\mathfrak{M}_{0}$ is a trivial module in which $m a \equiv 0$. The module $\mathfrak{M}_{1}$ is unital.

Now let $\mathfrak{M}$ be unital and let $1=\sum_{i}^{r} e_{i}$ where the $e_{i}$ are orthogonal idempotent elements. Then we have the Peirce decomposition

$$
\mathfrak{M}=\sum_{i, j=1}^{r} \oplus \mathfrak{M}_{i s}
$$

where

$$
\begin{aligned}
& \mathfrak{M}_{i i}=\left\{m_{i i} \mid m_{i i} e_{i}=m_{i i}\right\} \\
& \mathfrak{M}_{i j}=\mathfrak{M}_{j i}=\left\{m_{i j} \mid m_{i j} e_{j}=\frac{1}{2} m_{i j}=m_{i j} e_{i}\right\}, \quad i \neq j .
\end{aligned}
$$

This follows directly from the well-known Peirce decomposition of a Jordan algebra applied to the split null extension $\mathscr{F}=\mathfrak{\Im} \oplus \mathfrak{M} .^{8)}$ We have $\mathfrak{F}=\sum \oplus \mathfrak{F}_{i j}$ and $\mathfrak{M}_{i j}=\mathfrak{F}_{i j} \cap \mathfrak{M}$. We recall also the following well-known relations

8) Cf. Albert [2], p. 558 . 


$$
\begin{aligned}
\mathfrak{F}_{i i}^{2} \leq \mathfrak{F}_{i i}, \quad \mathfrak{F}_{i i} \mathfrak{F}_{i j} & =\mathfrak{F}_{i j} \mathfrak{F}_{i i} \leq \mathfrak{F}_{i j}, \quad \mathfrak{F}_{i j}^{2} \leq \mathfrak{F}_{i i}+\mathfrak{F}_{j j} \\
\mathfrak{F}_{i j} \mathfrak{F}_{j k} \leq \mathfrak{F}_{i k} &
\end{aligned}
$$

for $i, j, k \neq$. All other products of the terms in the Peirce decomposition are 0 . These relations imply relations connecting the terms of the Peirce decomposition of $\Im$ relative to the $e_{i}$ ard those of the decomposition of $\mathfrak{M}$.

Assume next that $\Im=\sum_{1}^{r} \oplus \Im_{i}$ where the $\Im_{i}$ are ideals and $1=\sum u_{i}, u_{i} \in \mathfrak{\Im}_{i}$. We consider first a special module $\mathfrak{M}=\mathfrak{M}_{\frac{1}{2}}$ and we note that we can decompose such a module as a direct sum of submodules $\mathfrak{M}_{i}$ such that $\mathfrak{M}_{i}$ is annihilated by all the $\left.\mathfrak{\Im}_{j} \neq \mathfrak{\Im}_{i} \cdot{ }^{9}\right)$ Suppose next that $\mathfrak{M}$ is unital. Then the $\mathfrak{\Im}_{i j}=0$, for $i \neq j$, in the Peirce decomposition of $\mathfrak{\Im}$ relative to the $e_{i}=u_{i}$ and

$$
\mathfrak{M}_{i j,} \mathfrak{F}_{i} \subseteq \mathfrak{M}_{i j}, \quad \mathfrak{M}_{i, j} \mathfrak{\Im}_{k}=0 \quad \text { if } \quad k \neq i, j .
$$

Hence the $\mathfrak{M}_{i \text { g }}$ are submodules of $\mathfrak{M}$. Also, it is clear that $\mathfrak{M}_{i i}$ is a unital $\mathfrak{\Im}_{i}$-module which is annihilated by the other $\mathfrak{\Im}_{j}$. In $\mathfrak{M M}_{i j}, i \neq j$, we have $R_{u_{i}}=\frac{1}{2} 1=R_{u_{j}}$. Hence this module is a special $\Im_{i^{-}}$and $\Im_{j}$-module, annihilated by all the other $\mathfrak{\Im}_{k}$. If we take $m=m_{i j}$ in $\mathfrak{M}_{i j}$ and the elements $u_{i}, a_{i} \in \mathfrak{\Im}_{i}, a_{j} \in \mathfrak{\Im}_{j}$ in the defining equations (5), we obtain $m_{i j} a_{i} a_{j}=m_{i j} a_{j} a_{i}$. Hence $\mathfrak{M}_{i j}$ is a sum of two commuting special modules one of which is essentially a $\mathfrak{\Im}_{i}$-module, the other essentially a $\mathfrak{\Im}_{\mathfrak{j}}$-module. These considerations reduce the study of a unital $\Im$-module to that of sums of these special $\mathfrak{\Im}_{i}$-modules and to unital $\mathfrak{\Im}_{\mathfrak{j}}$-modules (the $\mathfrak{M}_{i i}$ ).

\section{Matrix Algebras}

\section{Canonical involutions in matrix algebras}

In the theory of Jordan algebras we shall have to consider matrix algebras over alternative algebras and certain types of involutions in these algebras. A number of the results which will be required are valid also for matrix algebras over arbitrary non-associative algebras. Hence we shall begin our considerations with these.

Let $\mathfrak{D}$ be an arbitrary algebra with an identity 1 and let $\mathfrak{D}_{n}$ denote the algebra of $n \times n$ matrices with entries in $\mathfrak{D}$. If, as usual, we let $e_{i j}$ be the matrix with a 1 in the $(i, j)$ position and 0 's elsewhere then

9) Cf. [15], p. 146. 


$$
e_{i j} e_{k l}=\delta_{3 k} e_{i l}, \quad \sum_{1}^{n} e_{i i}=1,
$$

$i, j, k, l=1, \cdots, n$. It is also immediate that the $e_{i j}$ are in the nucleus, that is, in the subalgebra of elements $a$ which associate with the pairs $x, y$ in the sense that

$$
A(a, x, y)=A(x, a, y)=A(x, y, a)=0 .
$$

If $\mathfrak{A}$ is any non-associative algebra with an identity element, a system of $n^{2} e_{i j}$ in the nucleus of $\mathfrak{A}$ satisfying (28) is called a set of matrix units in $\mathfrak{A}$.

PROPOSITion 7.1. If $\mathfrak{A}$ is a (non-associative) algebra with an identity and $\left\{e_{i j}\right\}$ is a set of $n^{2}$ matrix units in $\mathfrak{A}$, then $\mathfrak{A} \cong \mathfrak{D}_{n}$ where $\mathfrak{D}$ is the subalgebra of elements of $\mathfrak{A}$ which commute with all the $e_{i j}$.

The usual proof of the associative special case goes over without change. We remark that the associativity of the $e_{i j}$ with all $x, y$ is needed to prove that the subset $\mathscr{D}$ of elements commuting with the $e_{i j}$ is a subalgebra. In the sequel we identify $\mathfrak{A}$ with $\mathfrak{D}_{n}$ and we write $\mathfrak{A}=\mathfrak{D}_{n}$.

Suppose now that the algebra $\mathfrak{D}$ has an involution $d \rightarrow \bar{d}$ (that is, an anti-isomorphism of period two). Then we can define an involution $a \rightarrow a^{*}$ in $\mathfrak{A}$ by setting $a^{*}=\sum \bar{d}_{i j} e_{j i}$ for $a=\sum d_{i j} e_{i j}, d_{i j} \in \mathcal{D}$. An involution of this type in $\mathfrak{U}=\mathfrak{D}_{n}$ will be called standard. More generally, let $\gamma_{1}=1, \gamma_{2}, \cdots, \gamma_{n}$ be self-adjoint $\left(\bar{\gamma}_{i}=\gamma_{i}\right)$ elements in the nucleus of $\mathfrak{D}$ having inverses, then we have the involution

$$
a=\sum d_{i j} e_{i j} \rightarrow \sum \gamma_{j}^{-1} \bar{d}_{i j} \gamma_{i} e_{j i}
$$

in $\mathfrak{D}_{n}$. Such an involution is called canonical. We have the following characterization.

Proposition 7.2. Let $\mathfrak{A}$ be an algebra with an identity element 1 which possesses an involution $a \rightarrow a^{*}$. Then $\mathfrak{A}=\mathfrak{D}_{1 b}$ and $a \rightarrow a^{*}$ is canonical (standard) if and only if $\mathfrak{A}$ possesses a set of $n^{2}$ matrix units $\left\{e_{i j}\right\}$ such that

$$
e_{i i}^{*}=e_{i i}, \quad i=1, \cdots, n \quad\left(e_{i j}^{*}=e_{j i}, \quad i, j=1, \cdots, n\right) .
$$

The proof of the associative special case given by Jacobson and Rickart [23] carries over without change for the canonical involution. This implies the result for standard involutions.

We consider now the Jordan structure of $\mathfrak{A}$. Thus we introduce the Jordan composition $\{x y\}=x y+y x$. This is, of course, commutative 
but it need not satisfy the second Jordan identity. Nevertheless, we denote the system $\mathfrak{A}$ relative to the given addition and scalar multiplication and the Jordan composition $\{x y\}$ as $\mathfrak{A}_{J}$. Suppose that $\mathfrak{U}$ has an involution $a \rightarrow a^{*}$. Then the subset $\mathfrak{S}$ of self-adjoint elements of $\mathfrak{A}$ is a subalgebra of $\mathfrak{A}_{J}$. We consider now the case in which $\mathfrak{U}=\mathfrak{D}_{n}$ and the involution is given by (29). We assume also that the characteristic of the base field is $\neq 2$.

A subset of $\mathfrak{A}$ or $\mathfrak{D}$ will be called self-adjoint if it is mapped into itself by the given involutions in these rings. An element $s$ is called skew if $s^{*}=-s$. Let $\mathfrak{S}$ denote the set of skew elements in $\mathfrak{A}$. If $\mathfrak{B}$ and $\mathbb{C}$ are subsets of an algebra, then we write [B্] for the set of sums $\sum[b c], b \in \mathfrak{B}, c \in \mathfrak{C}$ and $\{\mathfrak{B} \mathfrak{C}\}$ for the set of sums $\sum\{b c\}$. One verifies that if $\mathfrak{S}$ and $\mathfrak{S}$ are defined as before, then $[\mathfrak{S} \mathfrak{S}] \subseteq \mathfrak{S}$, $[\mathfrak{S}] \subseteq \mathfrak{S},[\mathfrak{S} \mathfrak{\mathfrak { C }}] \subseteq \mathfrak{S},\{\mathfrak{S} \mathfrak{S}\} \subseteq \mathfrak{S},\{\mathfrak{S} \mathfrak{S}\} \mathfrak{S}$.

PROPOSITION 7.3. Let $\mathfrak{A}=\mathfrak{D}_{n}, n \geq 3$, have a canonical involution where $\mathfrak{D}$ is any (non-associative) algebra with an identity. Let $\mathfrak{g}$ and $\mathfrak{S}$ be as above and let $\mathfrak{D}^{+}$and $\mathfrak{D}^{-}$, respectively, denote the sets of self-adjoint and skew elements of $\mathfrak{D}$. Then the dimensionality

$$
\operatorname{dim}(\mathfrak{S}-[\mathfrak{S} \mathfrak{S}]) \leq \operatorname{dim}\left(\mathfrak{D}^{-}-\left(\left[\mathfrak{D}^{+} \mathfrak{D}^{+}\right]+\left[\mathfrak{D}^{-} \mathfrak{D}^{-}\right]\right)\right)
$$

so that $\mathfrak{S}=[\mathfrak{S} \mathfrak{S}]$ if $\mathfrak{D}^{-}=\left[\mathfrak{D}^{-} \mathfrak{D}^{-}\right]+\left[\mathfrak{D}^{+} \mathfrak{D}^{+}\right]$. In any case

$$
\mathfrak{S}=[\mathfrak{S} \mathfrak{C}]+\{[\mathfrak{S} \mathfrak{S}], \mathfrak{C}\} \text {. }
$$

so that $\mathfrak{A}=\mathfrak{S}+[\mathfrak{S} \mathfrak{2}]+\{[\mathfrak{S} \mathfrak{2}], \mathfrak{L}\}$.

Proof. The elements of $\mathfrak{S}$ are sums of elements of the form $d e_{i j}-\gamma_{j}^{-1} \bar{d}_{\gamma_{i}} e_{j i}$. Assume $i, j, k \neq$ and form the commutator of $d e_{i j}+\gamma_{j}^{-1} \bar{d}_{\gamma_{i}} e_{j i}$ with $e_{j k}+\gamma_{k}^{-1} \gamma_{j} e_{k j}$ to obtain $d e_{i k}-\gamma_{k}^{-1} \bar{d}_{\gamma_{i}} e_{k i}$. This shows that all the skew elements of the form $d e_{i j}-\gamma_{j}^{-1} \bar{d}_{\gamma_{i}} e_{j i}, i \neq j$, are in [S్E]. We note next that

$$
\left(d-\gamma_{i}^{-3} \bar{d} \gamma_{i}\right) e_{i i}-\left(d-\gamma_{j}^{-1} \bar{d} \gamma_{j}\right) e_{j j}=\left[d e_{i j}+\gamma_{j}^{-1} \overline{d_{\gamma_{i}}} e_{j i}, e_{j i}+\gamma_{i}^{-1} \gamma_{j} e_{i j}\right]
$$

is in [SאS]. This shows that if we supplement [S్S] with the subspace of elements $q e_{11}, q \in \mathfrak{D}^{-}$, then we obtain the whole of $\mathfrak{S}$. On the other hand, if $p_{1}, p_{2} \in \mathfrak{D}^{+}$then $\left[p_{1} p_{2}\right] e_{11}=\left[p_{1} e_{11}, p_{2} e_{11}\right] \in[\mathfrak{S} \mathfrak{S}]$ and if $q_{1}=d_{1}-\overline{d_{1}}, q_{2}=d_{2}-\overline{d_{2}} \in \mathfrak{D}^{-}$then

$$
\begin{gathered}
{\left[q_{1} q_{2}\right] e_{11}=\left[\left[d_{1} e_{1 j}+\gamma_{j}^{-1} \overrightarrow{d_{1}} e_{j_{1}}, e_{j_{1}}+\gamma_{j} e_{1 j}\right],\right.} \\
\left.\left[d_{2} e_{1 k}+\gamma_{k}^{-1} \bar{d}_{2} e_{k_{1}}, e_{k_{1}}+\gamma_{k} e_{1 k}\right]\right] .
\end{gathered}
$$

if $1, j, k$ are $=$. Since the $\gamma_{j}, \gamma_{k}$ and $e_{i j}$ are in the nucleus we can use the Jacobi identity to show that the right hand side is in 


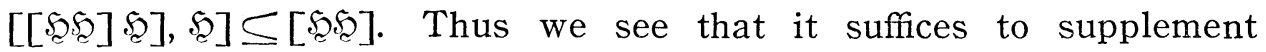
[\$2] by a subspace of elements $q e_{11}$ where $q$ is chosen in a complement of $\left[\mathfrak{D}^{+} \mathfrak{D}^{+}\right]+\left[\mathfrak{D}^{-} \mathfrak{D}^{-}\right]$in $\mathfrak{D}^{-}$. This proves the first statement. To prove the second we note that

$$
\frac{1}{2}\left\{e_{11},(d-\bar{d}) e_{11}-\left(d-\gamma_{j}^{-1} \bar{d}_{\gamma_{j}}\right) e_{j j}\right\}=(\bar{d}-d) e_{11} .
$$

Hence the addition of the space of these elements gives $\mathfrak{S}$.

Evidently, Prop. 7.2 implies that the subalgebra of $\mathfrak{A}$ generated by $\mathfrak{K}$ is $\mathfrak{A}$.

We shall now introduce certain notations which will be useful throughout this section. If $d \in \mathscr{D}$ and $i, j=1,2, \cdots, n$, then we set

$$
d[i j]=d e_{i j}+\gamma_{j}^{-1} \bar{d}_{\gamma_{i}} e_{j i}=d e_{i j}+\left(d e_{i j}\right)^{*} .
$$

Every element of $\mathfrak{S}$ is a sum of elements $d[i j]$. We have the Jordan relations

$$
\begin{aligned}
& \{d[i j], f[j k]\}=d f[i k] \\
& \{d[i j], f[j i]\}=d f[i i]+f d[j j]
\end{aligned}
$$

if $d, f \in \mathscr{D}$ and $i, j, k$ are $\neq$. Set

$$
u_{i j}=1[i j]=e_{i j}+\gamma_{j}^{-1} \gamma_{i} e_{j i}, \quad i \neq j .
$$

Theorem 7.1. Let $\mathfrak{A}=\mathfrak{D}_{n}$ have a canonical involution and assume $n \geqslant 3$. Let $\{\mathbb{5}\}$ be the collection of self-adjoint subalgebras of $\mathfrak{D}$ containing the $\gamma_{i}, \gamma_{i}^{-1}$ and $\{\mathfrak{\Im}\}$ the collection of self-adjoint ideals in $\mathfrak{D}$. Then the mappings $\mathfrak{F} \rightarrow \mathfrak{F}_{n} \cap \mathfrak{S}, \mathfrak{\Im} \rightarrow \mathfrak{S}_{n} \cap \mathfrak{S}$ are $1-1$ onto the set of subalgebras of $\mathfrak{S}$ containing the $u_{i j}, e_{i i}$ and onto the set of ideals of $\mathfrak{S}$ (relative to \{\}), respectively. The ideal $\Im$ satisfies $\mathfrak{\Im}^{2}=0$ if and only if the Jordan square $\left\{\mathfrak{S}_{n} \cap \mathfrak{S}_{2}, \mathfrak{\Im}_{n} \cap \mathfrak{S}\right\}=0$.

Proof. We consider first any subspace $\Omega$ of $\mathscr{S}$ which is closed

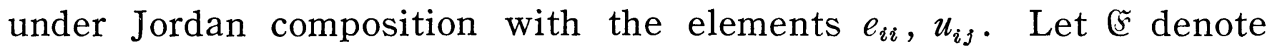
the subset of $\mathfrak{D}$ of elements which appear as entries for the matrices belonging to $\Re$. Let $k=\sum d_{i j} e_{i j}, d_{i j} \in \mathfrak{D}$, be in $\Re$. Since

$$
\left\{\left\{k e_{i i}\right\} e_{i i}\right\}-\left\{k e_{i i}\right\}=2 e_{i i} k e_{i i},
$$

$e_{i i} k e_{i i}=d_{i i} e_{i i} \in \Re$. If $i \neq j$ then $d_{i i}[i j]=\left\{d_{i i} e_{i i}, u_{i j}\right\} \in \Re$. Also

$$
\left\{\left\{e_{i i} k\right\} e_{j j}\right\}=d_{i j} e_{i j}+d_{j i} e_{j i} \in \Re \text {. }
$$

Since $\Re \subseteq \mathfrak{S}$, this element is $d_{i j}[i j]$. Thus we see that $\left[\begin{array}{c}5 \\ \text { can be }\end{array}\right.$ characterized as the set of elements $d \in \mathscr{D}$ such that $d[i j] \in \Re$ for 
some $i \neq j$. Since $d[k j]=\left\{u_{k i}, d[i j]\right\}$ and $d[i k]=\left\{d[i j], u_{j k}\right\}$ for $i, j, k=1=, d[k l] \in \Re$ for every $d \in[\leftarrow$ and $k=1=l$. This implies that $[5$ is a subspace of $\mathfrak{D}$. Since

$$
2 d[i i]=\left\{\left\{d[i j], u_{s i}\right\} e_{i i}\right\},
$$

$d[i i] \in \Re$ for all $i$ and all $d \in \mathfrak{F}$. Evidently $\Re \leq \mathfrak{S} \cap \mathfrak{F}_{n}$. On the other hand, if $\sum d_{i j} e_{i j} \in \mathfrak{S}$ and the $d_{i j} e_{i j} \in \mathfrak{F}$, then $\sum d_{i j}=\sum_{i<j} d_{i j}[i j]+$ $\frac{1}{2} \sum_{i} d_{i i}[i i] \in \Re$. Hence $\Re=\mathfrak{S} \cap \mathfrak{F}_{\mathfrak{B}}$. Now assume that $\Re$ is a subalgebra of $\mathfrak{L}$ containing the $u_{i j}$. Then $\Omega$ satisfies our conditions and so $\Re=\mathfrak{C}_{\mathfrak{B}} \cap \mathfrak{F}_{n}$ where $\mathbb{F}$ is defined above. It is clear from (31) that $\mathbb{F}$ is a subalgebra of $\mathfrak{D}$. Since $u_{i 1}^{2}=\gamma_{i}\left(e_{11}+e_{i \dot{i}}\right)$ and $u_{1 i}^{2}=\gamma_{i}^{-1}\left(e_{11}+e_{i i}\right), i>1$, (5 contains the elements $\gamma_{i}$ and $\gamma_{i}^{-1}$. Since

$$
d[i j]=\gamma_{j}^{-1} \bar{d}_{i}[j i],
$$

it is clear that $[5$ is self-adjoint. It is now easy to conclude the proof of the first assertion of the theorem. The second is obtained in exactly the same way. The last statement is an immediate consequence of (31).

Let the $e_{i i}$ and $u_{i j}, i \neq j$, be defined as before. We shall now list a number of Jordan relations connecting these elements, as follows:

$$
\begin{aligned}
& \left\{e_{i i} e_{i i}\right\}=2 e_{i i}, \\
& \left\{e_{i i} u_{i j}\right\}=u_{i j}=\left\{u_{i j} e_{j j}\right\} \\
& \left\{u_{i j} u_{j k}\right\}=u_{i k} \\
& \left\{u_{i j} u_{j i}\right\}=2\left(e_{i i}+e_{j j}\right)=\frac{1}{4}\left\{\left\{u_{i j} u_{i j}\right\}\left\{u_{j i} u_{j i}\right\}\right\} \\
& \left\{\left\{u_{i j} u_{i j}\right\} u_{j i}\right\}=4 u_{i j},
\end{aligned}
$$

if $i, j, k$ are $\equiv$. All the other Jordan products of these elements with the exception of $\left\{u_{i j} u_{k j}\right\}$ are 0 .

LEMMA. Let $x$ and $y$ be elements of an associative algebra with an identity such that $x y+y x=2=x^{2} y^{2}+y^{2} x^{2}, x^{2} y+y x^{2}=2 x$. Then $x y=1=y x$.

Proof. Our first relation gives $x^{2} y+x y x=2 x=x y x+y x^{2}$. Since $x^{2} y+y x^{2}=2 x$, this implies $x^{2} y=x y x=y x^{2}=x$. Then $x^{2} y^{2}=y x^{2} y$ $=y^{2} x^{2}=x y$. Since $x^{2} y^{2}+y^{2} x^{2}=2$ this gives $x^{2} y^{2}=1=y^{2} x^{2}$. Hence $x y=1$ and $y x=1$.

The essence of the situation we are considering is that we have a subalgebra of an $\mathfrak{U}_{J}$ containing $e_{i i}, u_{i j}$ satisfying the Jordan rela- 
tions we have listed. Thus we have the following result.

Theorem 7.2. Let $\mathfrak{N}$ be a (non-associative) algebra with an element 1 and let $\mathfrak{S}$ be a subalgebra of $\mathfrak{N}_{J}$ containing elements $e_{i i}, u_{i j}, i \neq j$, in the nucleus satisfying (35). Assume, moreover, that all the other Jordan products of the $e$ 's and $u$ 's are 0 with the exception of $\left\{u_{i, j} u_{k,}\right\}$, that $\sum e_{i i}=1$ and that $n \geqslant 3$. Then $\mathfrak{A}=\mathfrak{D}_{n}$. Let $\mathfrak{D}^{\prime}$ be anti-isomorphic to D under a mapping $d \rightarrow d^{\prime}$, whence $d_{1}+d_{2}{ }^{\prime} \rightarrow d_{2}+d_{1}{ }^{\prime}$ is an involution in $\mathfrak{D} \oplus \mathfrak{D}^{\prime}$. Then $\mathfrak{S}$ is isomorphic to the subalgebra of self-adjoint elements of an $\widetilde{F}_{n J}$ where $\mathfrak{F}$ is a self-adjoint subalgebra of $\mathfrak{D} \oplus \mathfrak{D}^{\prime}$ and the involution in $\mathfrak{\mho}_{n J}$ is canonical.

Proof. The first relation shows that the $e_{i i}$ are idempotent and our assumption is that these elements are orthogonal: $e_{i i} e_{j j}=0, i=j$. The second line in (35) gives $e_{i i} u_{i j} e_{i i}=0=e_{j j} u_{i j} e_{j j}$ and

$$
e_{i i} u_{i j} e_{j j}=u_{i j} e_{j j}=e_{i i} u_{i j}, \quad e_{j j} u_{i j} e_{i i}=e_{j j} u_{i j}=u_{i j} e_{i i} .
$$

Hence $u_{i j}=e_{i i} u_{i j} e_{j j}+e_{j j} u_{i j} e_{i i}$ and $u_{i j}=\left(e_{i i}+e_{j j}\right) u_{i j}\left(e_{i i}+e_{j \jmath}\right)$. This implies

$$
\left(e_{i i}+e_{j \jmath}\right) u_{i j}=u_{i j}=u_{i j}\left(e_{i i}+e_{j j}\right) \text {. }
$$

Thus $e_{i i}+e_{j j}$ acts as the identity for $u_{i j}$ and $u_{j i}$. The last two lines of (35) and the lemma now imply

$$
u_{i j} u_{j i}=e_{i i}+e_{j \jmath}=u_{j i} u_{i j} .
$$

Now set $g_{1 i}=e_{11} u_{1 i} e_{i i}, g_{i 1}=e_{i i} u_{i 1} e_{11}, i=2, \cdots, n$. Then

$$
\begin{aligned}
& g_{1 i} g_{i 1}=e_{11} u_{1 i} u_{i 1} e_{11}=e_{11}\left(e_{11}+e_{i i}\right) e_{11}=e_{11} \\
& g_{i 1} g_{1 i}=e_{i i} u_{i 1} u_{1 i} e_{i i}=e_{i i}\left(e_{11}+e_{i i}\right) e_{i i}=e_{i i} .
\end{aligned}
$$

Hence if we put $g_{i i}=e_{i i}, g_{i j}=g_{i 1} g_{1 j}$ for $1, i, j \neq$, then the $g_{k l}$ thus defined form a set of $n^{2}$ matrix units. It follows that $\mathfrak{A}=\mathfrak{D}_{n}$ where $\left(\mathfrak{D}\right.$ is the subalgebra of $\mathfrak{U}$ of elements which commute with the $g_{k l}$. This proves the first assertion. Also, since for $i>1, u_{1 i}=e_{11} u_{1 i} e_{i i}$ $+e_{i i} u_{1 i} e_{11}, u_{1 i}=\gamma_{1 i} g_{1 i}+\gamma_{i_{1}} g_{i_{1}}$ where the $\gamma$ 's are in the nucleus of $\mathfrak{D}$. Since $g_{1 i}=e_{11} u_{1 i} e_{i i}, u_{1 i}=g_{1 i}+\gamma_{i}^{\prime} g_{i_{1}}, \gamma_{i}^{\prime}$ in the nucleus. Similarly $u_{i_{1}}=g_{i_{1}}+\gamma_{i} g_{1 i}, \gamma_{i}$ in the nucleus. Since $u_{i_{1}} u_{1 i}=e_{11}+e_{i i}=u_{1 i} u_{i_{1}}$, $\gamma_{i}^{\prime}=\gamma_{i}^{-1}$. Set $\gamma_{1}=\gamma_{1}^{\prime}=1$, since $u_{i j}=\left\{u_{i_{1}} u_{1 j}\right\}$ for $1, i, j \neq=$,

$$
u_{i j}=g_{i j}+\gamma_{j}^{-1} \gamma_{i} g_{j i}
$$

holds for all $i, j \neq$.

Let $x \in \mathfrak{S}$. Then $e_{i i} x e_{i i}=\frac{1}{2}\left\{\left\{e_{i i} x\right\} e_{i i}\right\}-\frac{1}{2}\left\{e_{i i} x\right\}$ and, if $i \neq j$, 
$e_{i i} x e_{j j}+e_{j j} x e_{i i}=\left\{\left\{e_{i i} x\right\} e_{j j}\right\} \in \mathfrak{S}$. Set $x_{i i}=e_{i i} x e_{i i}, x_{i j}=e_{i i} x e_{j j}+e_{j j} x e_{i i}=x_{j i}$ for $i \neq j$ and $\mathfrak{S}_{k l}=\left\{x_{k l} \mid x \in \mathfrak{S}_{\mathfrak{g}}\right\}$. Then $\mathfrak{S}=\sum_{k \leq l} \oplus \mathfrak{S}_{k l}$. We can write $x_{i i}=\lambda_{i i}\left(x_{i i}\right) g_{i i}, x_{i j}=\lambda_{i j}\left(x_{i j}\right) g_{i j}+\lambda_{j i}\left(x_{i j}\right) g_{j i}, i \neq j$, where $\lambda_{i i}\left(x_{i i}\right), \lambda_{i j}\left(x_{i j}\right)$, $\lambda_{j i}\left(x_{i j}\right) \in \mathfrak{D}$. Let $\mathfrak{D}^{\prime}$ be anti-isomorphic to $\mathfrak{D}$ under a mapping $d \rightarrow d^{\prime}$. Then $\mathfrak{D} \oplus \mathfrak{D}^{\prime}$ has the involution $d_{1}+d_{2}{ }^{\prime} \rightarrow d_{2}+d_{1}^{\prime}, d_{i} \in \mathfrak{D}$. Let $\mathfrak{F}=\left\{\lambda_{12}\left(x_{12}\right)\right.$ $\left.+\left(\gamma_{2} \lambda_{21}\left(x_{12}\right)\right)^{\prime} \mid x_{12} \in \mathfrak{L}_{12}\right\}$. This is a subgroup of the additive group of $\mathfrak{D} \oplus \mathfrak{D}^{\prime}$. Take $x_{12}, y_{12} \in \mathfrak{S}_{12}$ and calculate

$$
\frac{1}{2}\left\{\left\{x_{12} u_{23}\right\}\left\{y_{12} u_{31}\right\}\right\}=\lambda_{12}\left(x_{12}\right) \lambda_{12}\left(y_{12}\right) g_{12}+\lambda_{21}\left(y_{12}\right) \gamma_{2} \lambda_{21}\left(x_{12}\right) g_{21} \text {. }
$$

This shows that

$$
\begin{aligned}
& \lambda_{12}\left(x_{12}\right) \lambda_{12}\left(y_{12}\right)+\left(\gamma_{2} \lambda_{21}\left(y_{12}\right) \gamma_{2} \lambda_{21}\left(x_{12}\right)\right)^{\prime} \\
= & \left(\lambda_{12}\left(x_{12}\right)+\left(\gamma_{2} \lambda_{21}\left(x_{12}\right)\right)^{\prime}\right)\left(\lambda_{12}\left(y_{12}\right)+\left(\gamma_{2} \lambda_{21}\left(y_{12}\right)\right)^{\prime}\right) \in \mathfrak{F} .
\end{aligned}
$$

Hence $\mathfrak{F}$ is a subring of $\mathfrak{D} \oplus \mathfrak{D}^{\prime}$. We calculate next

$$
\left\{\left\{\left\{x_{12} u_{23}\right\} u_{12}\right\} u_{31}\right\}=\gamma_{2} \lambda_{21}\left(x_{12}\right) g_{12}+\gamma_{2}^{-1} \lambda_{12}\left(x_{12}\right) g_{21},
$$

which shows that $\gamma_{2} \lambda_{21}\left(x_{12}\right)+\left(\lambda_{12}\left(x_{12}\right)\right)^{\prime} \in \mathfrak{F}$. Thus $\mathfrak{F}$ is self-adjoint.

We write next the following relations

$$
\begin{aligned}
& \left\{x_{1 j} u_{j 2}\right\}=\lambda_{1 j}\left(x_{1 j}\right) g_{12}+\gamma_{2}^{-1} \gamma_{j} \lambda_{j 1}\left(x_{1 j}\right) g_{21}, \quad j \neq 2 \\
& \left\{x_{2 j} u_{1 j}\right\}=\lambda_{j 2}\left(x_{2 j}\right) g_{12}+\lambda_{2 j}\left(x_{2 j}\right) \gamma_{j}^{-1} g_{21}, \quad j \neq 1 \\
& \left\{\left\{x_{i j} u_{1 i}\right\} u_{j 2}\right\}=\lambda_{i j}\left(x_{i j}\right) g_{12}+\gamma_{2}^{-1} \gamma_{j} \lambda_{j i}\left(x_{i j}\right) \gamma_{i}^{-1} g_{21}, \quad i \neq 1, j \neq 1,2 .
\end{aligned}
$$

If we recall that $\gamma_{1}=1$, these imply that

$$
\lambda_{i j}\left(x_{i j}\right)+\left(\gamma_{j} \lambda_{j i}\left(x_{i j}\right) \gamma_{i}^{-1}\right)^{\prime} \in \mathfrak{F}
$$

for all $i \leqslant j$. It is easy to see that $\gamma_{i} g_{11}, \gamma_{i}^{-1} g_{11} \in \mathcal{S}_{\mathcal{C}}$. If we set these $=x_{11}$ we see that $\Gamma_{i} \equiv \gamma_{i}+\gamma_{i}{ }^{\prime}, \Gamma_{i}^{-1}=\gamma_{i}^{-1}+\left(\gamma_{i}{ }^{\prime}\right)^{-1} \in \mathfrak{F}$.

We now define for $i \leqslant j$

$$
\begin{aligned}
& M_{i j}\left(x_{i j}\right)=\lambda_{i j}\left(x_{i j}\right)+\left(\gamma_{j} \lambda_{j i}\left(x_{i j}\right) \gamma_{i}^{-1}\right)^{\prime} \\
& M_{j i}\left(x_{i j}\right)=\lambda_{j i}\left(x_{i j}\right)+\left(\gamma_{i} \lambda_{i j}\left(x_{i j}\right) \gamma_{j}^{-1}\right)^{\prime} .
\end{aligned}
$$

Then $M_{j i}\left(x_{i j}\right)=\Gamma_{j}^{-1} \overline{M_{i j}\left(x_{i j}\right)} \Gamma_{i}$ where the bar refers to the involution in $\mathfrak{F}$. We can verify that

$$
\begin{aligned}
& M_{i k}\left(\left\{x_{i j} y_{j k}\right\}\right)=M_{i j}\left(x_{i j}\right) M_{j k}\left(y_{j k}\right), \quad i<j<k \\
& M_{i j}\left(\left\{x_{i i} y_{i j}\right\}\right)=M_{i i}\left(x_{i i}\right) M_{i j}\left(y_{i j}\right), \quad i<j \\
& M_{i i}\left(\left\{x_{i i} y_{i i}\right\}\right)=\left\{M_{i i}\left(x_{i i}\right) M_{i i}\left(y_{i i}\right)\right\} .
\end{aligned}
$$

We now introduce the matrix algebra $\widetilde{F}_{n}$ with matrix units $G_{i j}$, 
$i, j=1,2, \cdots, n$ and we consider the linear mapping of $\mathfrak{C}$ into $\mathfrak{F}_{n}$ such that

$$
\begin{aligned}
& x_{i j} \rightarrow M_{i j}\left(x_{i j}\right) G_{i j}+M_{j i}\left(x_{i j}\right) G_{j i}, \quad i<j \\
& x_{i i} \rightarrow M_{i i}\left(x_{i i}\right) G_{i i} .
\end{aligned}
$$

It is clear that this is $1-1$. We can now verify directly that

$$
x_{i j}^{2} \rightarrow\left(M_{i j}\left(x_{i j}\right) G_{i j}+M_{j i}\left(x_{i j}\right) G_{j i}\right)^{2} .
$$

This relation together with $(39)-(42)$ shows that we have an isomorphism of $\mathfrak{S}$ into $\mathfrak{F}_{n J}$. It is easy to verify that the image under this isomorphism is the set of self-adjoint elements relative to the canonical involution in $\mathfrak{F}_{n}$ defined by $\Gamma=\operatorname{diag}\left\{\Gamma_{1}, \Gamma_{2}, \cdots, \Gamma_{n}\right\}$. This completes the proof.

We return now to the original notation: $\mathfrak{A}=\mathfrak{D}_{n}$ with a canonical involution given by $\operatorname{diag}\left\{\gamma_{1}, \gamma_{2}, \cdots, \gamma_{n}\right\}, \gamma_{1}=1$, $\mathfrak{S}$ the subalgebra of $\mathfrak{A}_{J}$ of self-adjoint elements.

Theorem 7.3. Let $\mathfrak{A}=\mathfrak{D}_{n}$ have a canonical involution and let $\mathfrak{S}$ be the subalgebra of $\mathfrak{A}_{J}$ of self-adjoint elements. Assume $n \geqslant 3$. Let $S$ be a homomorphism of $\mathfrak{S}$ into an algebra $\mathfrak{B}_{J}$ such that the $e_{i i}^{S}$, $u_{i j}^{S}$ are in the nucleus of the subalgebra of $\mathfrak{B}$ generated by the image of $\mathfrak{S}$ under S. Then $S$ can be extended in one and only one way to a homomorphism of $\mathfrak{U}$ into $\mathfrak{B}$.

Proof. We may as well assume that the subalgebra of $\mathfrak{B}$ generated by $\mathfrak{S C}^{s}$ is $\mathfrak{B}$ itself. Then $1^{s}$ is the identity element of $\mathfrak{B}$ and the $u_{i j}^{S}$, satisfy the condition (35). We may therefore use these to construct matrix units in $\mathfrak{B}$ and obtain a representation of $\mathfrak{B}$ as $\mathfrak{F}_{n}$. The remainder of the proof can be carried out exactly as in the associative special case considered by Jacobson and Rickart [23]. We remark that the uniqueness of the extended homomorphism is an immediate consequence of the fact the enveloping algebra (i. e. subalgebra of $\mathfrak{U}$ generated) of $\mathfrak{S}$ is $\mathfrak{A}$ itself.

An important feature of the present method is the complete arbitrariness of the algebra $\mathfrak{D}$. This permits a uniform treatment of a number of interesting special cases. We shall not attempt to list these but shall be content to call attention to one which will be needed later: the case of $\mathfrak{A}_{J}=\mathfrak{D}_{n_{J}}$ itself. ${ }^{10)}$ To obtain this algebra as an algebra $\mathfrak{S}$ we introduce the algebra $\mathfrak{D}^{\prime}$ anti-isomorphic to $\mathfrak{D}$ relative to the involution $d \rightarrow d^{\prime}$. As in the proof of Th. 7.2 we

10) A number of other examples are given in [23], pp. 311-313. 
define the involution $d_{1}+d_{2}^{\prime} \rightarrow d_{1}^{\prime}+d_{2}$ in the algebra $\mathfrak{D} \oplus \mathfrak{D}^{\prime}$ which we now denote as $\mathfrak{F}$. Let $\mathfrak{S}$ be the set of self-adjoint elements relative to the standard involution in $\mathfrak{F}_{n}$. Then it is easy to see that $\mathfrak{S}_{\mathfrak{C}}$ relative to \{\} is isomorphic to $\mathfrak{D}_{n J}$. Our results therefore apply to $\mathfrak{D}_{n J}$. In particular, Th. 7.3 holds. However, in this case, we can improve the inequality $n \geqslant 3$ to $n \geqslant 2$ and we can formulate the result in a slightly different manner as follows.

Theorem 7.4. Let $\mathfrak{D}$ be an arbitrary algebra with an identity and let $\mathfrak{A}$ be the matrix algebra $\mathfrak{D}_{n}, n \geqslant 2$. Let $S$ be a homomorphism of $\mathfrak{A}_{J}$ into any algebra $\mathfrak{B}_{J}$ such that the matrix units $e_{i j}$ are mapped into elements of the nucleus of the subalgebra $\mathfrak{c}$ of $\mathfrak{B}$ generated by $\mathfrak{A}^{\mathrm{s}}$. Then $\mathfrak{S}=\mathfrak{S}^{(1)} \oplus \mathfrak{S}^{(2)}$ where the $\mathfrak{S}^{(i)}$ are ideals such that the product of $S$ and the projection of $\mathbb{( S}$ onto $\mathfrak{F}^{(1)}$ is a homomorphism of $\mathfrak{A}$ onto $\mathfrak{C}^{(1)}$ while the product of $S$ and the projection onto $\mathfrak{C}^{(2)}$ is an anti-homomorphism of $\mathfrak{A}$ onto $\mathfrak{c}$.

Proof. It is easy to deduce this result for $n \geqslant 3$ from Th. 7.3. However, to include the case $n=2$ it seems to be necessary to employ an earlier direct method which has been used by Jacobson and Rickart in [22]. It is easy to see that the hypothesis of associativity used in [22] can be weakened to the one stated above.

\section{Exceptional Jordan algebras}

We shall now consider the following question: What are the conditions that an algebra $\mathfrak{S}$ of self-adjoint elements of $\mathfrak{U}=\mathfrak{D}_{n}$ relative to a canonical involution be a Jordan algebra? It is not hard to see that if $n \geqslant 4$ then $\mathfrak{S}$ will not satisfy the Jordan identities unless $\mathfrak{D}$ is associative (cf Th. 9.1). Hence the Jordan algebras $\mathfrak{S}$ obtained for $n \geqslant 4$ are all special. If $n=3$ it is easy to see that necessary conditions that $\mathfrak{S}$ is Jordan are that $\mathfrak{D}$ is alternative and that the selfadjoint elements relative to the involution in $\mathscr{D}$ belong to the nucleus. One might conjecture that these are also sufficient conditions. However, the calculations which seem to be required to settle this question are quite formidable. Hence we shall confine our attention to proving a weaker result which will be sufficient for our purposes. Thus we shall prove that $\mathfrak{S}$ is Jordan if $n=3$ and $\mathfrak{D}$ is alternative with an involution whose self-adjoint elements are in the center. For further simplification we assume also that the involution in $\mathfrak{A}$ is standard. ${ }^{11)}$

11) This restriction is not really necessary. 
Let $\mathfrak{D}_{0}$ denote the subspace of self-adjoint elements of $\mathfrak{D}$. If $a \in \mathfrak{D}$ then $a+\bar{a}$ and $a \bar{a} \in \mathscr{D}_{0}$. We denote the linear mappings $x \rightarrow \bar{x}$ and $x \rightarrow x+\bar{x}$ by $C$ and $T$ respectively.

Lemma. $\quad a \bar{a}=\bar{a} a, A(a, \bar{a}, b)=0, \quad(a b) T=(b a) T,((a b) c) T=(a(b c)) T$, $(a b)(c a)=a(b c a), a(b(a c))=(a b a) c$.

The first four of these are easily established using the properties of the involution. The last two are well-known identities due to Moufang [29] which are valid in any alternative ring.

We denote the elements of $\mathfrak{L}$ as

$$
x=\left(\begin{array}{lll}
\xi_{1} & x_{3} & \bar{x}_{2} \\
\bar{x}_{3} & \xi_{2} & x_{1} \\
x_{2} & \bar{x}_{1} & \xi_{3}
\end{array}\right), \quad \bar{\xi}_{i}=\xi_{i} .
$$

Thus we have a decomposition of $\mathfrak{S}$ as a direct sum of six subspaces, three of which are isomorphic to $\mathscr{D}$ and three of which are isomorphic to $\mathfrak{D}_{0}$. Hence we may identify $x$ with the vector $\left(\xi_{1}, \xi_{2}, \xi_{3}, x_{1}, x_{2}, x_{3}\right)$, $\xi_{i} \in \mathfrak{D}_{0}, x_{i} \in \mathfrak{D}$. Any linear transformation in $\mathfrak{S}$ can be represented by a $6 \times 6$ matrix $\left(L_{i j}\right)$ where $L_{i j}$ is a linear mapping of the $i$-th space in the sequence $\left(\mathscr{D}_{0}, \mathfrak{D}_{0}, \mathfrak{D}_{0}, \mathfrak{D}, \mathfrak{D}, \mathfrak{D}\right)$ into the $j$-th. The linear transformation is the one such that

$$
\begin{aligned}
& \xi_{i} \rightarrow \xi_{1} L_{1 i}+\xi_{2} L_{2 i}+\xi_{3} L_{3 i}+x_{1} L_{4 i}+x_{2} L_{5 i}+x_{3} L_{6 i} \\
& x_{i} \rightarrow \xi_{1} L_{1, i+3}+\xi_{2} L_{2, i+3}+\xi_{3} L_{3, i+3}+x_{1} L_{4, i+3}+x_{2} L_{5, i+3}+x_{3} L_{6, i+3} .
\end{aligned}
$$

This .can be indicated also by multiplication of $\left(\xi_{1}, \xi_{2}, \xi_{3}, x_{1}, x_{2}, x_{3}\right)$ on the right by $\left(L_{i j}\right)$. Now let $a=\left(\alpha_{1}, \alpha_{2}, \alpha_{3}, a_{1}, a_{2}, a_{3}\right), \alpha_{i} \in \mathscr{D}_{0}$, $a_{i} \in \mathcal{D}$. Then $\{x a\}=y=\left(\eta_{1}, \eta_{2}, \eta_{3}, y_{1}, y_{2}, y_{3}\right)$, where

$$
\begin{aligned}
& \eta_{i}=\left\{\xi_{i} \alpha_{i}\right\}+\left(x_{j} \bar{a}_{j}\right) T+\left(x_{k} \bar{a}_{k}\right) T \\
& y_{i}=\left(\xi_{j}+\xi_{k}\right) a_{i}+x_{i}\left(\alpha_{j}+\alpha_{k}\right)+\bar{x}_{k} \bar{a}_{j}+\bar{a}_{k} \bar{x}_{j}
\end{aligned}
$$

where $(i, j, k)$ takes on the cyclic permutations of $(1,2,3)$ and $T$ is the "trace" mapping defined before. Hence we can represent the linear transformation $R_{a}(x \rightarrow\{x a\})$ by the matrix

$$
\left(\begin{array}{llllll}
2 R_{\alpha_{1}} & 0 & 0 & 0 & R_{a_{2}} & R_{a_{3}} \\
0 & 2 R_{\alpha_{2}} & 0 & R_{a_{1}} & 0 & R_{a_{3}} \\
0 & 0 & 2 R_{\alpha_{3}} & R_{a_{1}} & R_{a_{2}} & 0 \\
0 & R_{\bar{a}_{1}} T & R_{\bar{a}_{1}} T & R_{\alpha_{2}+\alpha_{3}} & C R_{\bar{a}_{3}} & R_{a_{2}} C \\
R_{\bar{a}_{2}} T & 0 & R_{\bar{a}_{2}} T & R_{a_{3}} C & R_{\alpha_{1}+\alpha_{3}} & C R_{\bar{a}_{1}} \\
R_{\bar{a}_{3}} T & R_{\bar{a}_{3}} T & 0 & C R_{\bar{a}_{2}} & R_{a_{1}} C & R_{\alpha_{1}+\alpha_{3}}
\end{array}\right)
$$


where the $R$ 's in the matrix stand for right multiplications in $D$. Now let $b=\left(\beta_{i}, \beta_{2}, \beta_{3}, b_{1}, b_{2}, b_{3}\right), \beta_{i} \in \mathscr{D}_{0}, b_{i} \in \mathfrak{D}$. Then the matrix of $\left[R_{a} R_{b}\right]$ is the commutator of the matrices of $R_{a}$ and $R_{b}$. If we use the fact that self-adjoint elements are in the center and the preceding lemma, then we can see that the matrix of $\left[R_{a} R_{b}\right]$ is

$$
\left(\begin{array}{cccccc}
0 & 0 & 0 & 0 & -R_{c_{2}} & R_{c_{3}} \\
0 & 0 & 0 & R_{c_{1}} & 0 & -R_{c_{3}} \\
0 & 0 & 0 & -R_{c_{1}} & R_{c_{2}} & 0 \\
0 & -R_{\bar{c}_{1}} T & R_{\bar{c}_{1}} T & U_{1} & V_{3} & W_{2} \\
R_{\bar{c}_{2}} T & 0 & -R_{\bar{c}_{2}} T & W_{3} & U_{2} & V_{1} \\
-R_{\bar{c}_{3}} T & R_{\bar{c}_{3}} T & 0 & V_{2} & W_{1} & U_{3}
\end{array}\right)
$$

where

$$
\begin{gathered}
c_{i}=b_{i}\left(\alpha_{j}-\alpha_{k}\right)-a_{i}\left(\beta_{j}-\beta_{k}\right)+\left(\bar{a}_{k} \bar{b}_{j}-\bar{b}_{k_{i}} \bar{a}_{j}\right) \\
U_{i}=2\left(R_{\bar{a}_{i}} T R_{b_{i}}-R \bar{b}_{i} T R_{a_{i}}\right)+\left(L_{a_{k}} L_{\bar{b}_{k}}-L_{b_{k}} L_{\bar{a}_{k}}\right)+\left(R_{a_{j}} R \bar{b}_{j}-R_{b_{j}} R_{\bar{a}_{j}}\right) \\
V_{i}=C R_{d_{i}}+\left(R_{\bar{a}_{j}} T R \bar{b}_{k}-R \bar{b}_{j} T R_{a_{k}}\right)+\left(R_{a_{k}} L_{b_{j}}-R_{b_{k}} L_{\bar{a}_{j}}\right) \\
d_{i}=\left(\beta_{j}-\beta_{k_{k}}\right) \bar{a}_{i}-\left(\alpha_{j}-\alpha_{k}\right) \bar{b}_{i} \\
W_{i}=-C L_{d_{i}}+\left(R_{\bar{a}_{k}} T R_{b_{j}}-R \bar{b}_{k} T R_{a_{j}}\right)+\left(L_{a_{j}} R_{\bar{b}_{k}}-L_{b_{j}} R_{\bar{a}_{k}}\right) .
\end{gathered}
$$

Now set $b=a^{2}=\frac{1}{2}\{a a\}$. Then by $(44)$

$$
\begin{aligned}
\beta_{i} & =\left(\alpha_{i}^{2}+a_{j} \bar{a}_{j}+a_{k} \bar{a}_{k}\right) \\
b_{i} & =\left(\alpha_{j}+\alpha_{k}\right) a_{i}+\bar{a}_{k} \bar{a}_{i} .
\end{aligned}
$$

We are now ready to prove the following

Theorem 8.1. If $\mathfrak{D}$ is an alternative algebra with an involution such that the self-adjoint elements are in the center, then the subalgebra $\mathfrak{S}$ of $\mathfrak{U}_{J}$ of self-adjoint elements of $\mathfrak{U}=\mathfrak{D}_{3}$ relative to the standard involution is a Jordan algebra. ${ }^{12}$

Proof. We take the values of $\beta_{i}$ and $b_{i}$ from $(51)$ and substitute in $c_{i}, U_{i}, V_{i}$ and $W_{i}$. This gives

$$
\begin{aligned}
c_{i}= & \left(\alpha_{j}^{2}-\alpha_{k}^{2}\right) a_{i}+\bar{a}_{k} \bar{a}_{\mathfrak{j}}\left(\alpha_{j}-\alpha_{k}\right)-a_{i}\left(\alpha_{j}^{2}-\alpha_{k}^{2}+a_{k} \bar{a}_{k i}-a_{j} \bar{a}_{\mathfrak{j}}\right) \\
& +\bar{a}_{k}\left[\left(\alpha_{k}+\alpha_{i}\right) \bar{a}_{j}+a_{k} a_{i}\right]-\left[\left(\alpha_{i}+\alpha_{j}\right) a_{k}+a_{i} a_{j}\right] \bar{a}_{j}
\end{aligned}
$$

12) The only case of this result which seems to have been proved before is that of the exceptional Jordan algebra $M_{3}^{8}$ defined by Jordan, von Neumann and Wigner in [25]. Here the base field is that of the real numbers and the proof given by Albert in [1] does not generalize to the present case. The proof which we give here was communicated to us by R. D. Schafer. 


$$
\begin{aligned}
& =\bar{a}_{k}\left(a_{k} a_{i}\right)-a_{i}\left(a_{k} \bar{a}_{k}\right)+\left(a_{i} a_{j}\right) \bar{a}_{j}-a_{i}\left(a_{j} \bar{a}_{j}\right) \\
& =0
\end{aligned}
$$

by the Lemma. Next we have

$$
\begin{aligned}
U_{i}= & 2\left(R_{\bar{a}_{i}} T R_{\bar{a}_{k} \bar{a}_{j}}-R_{a_{j} a_{k}} T R_{a_{i}}\right) \\
& +\left(L_{a_{k}} L_{a_{i} a_{j}}-L_{\bar{a}_{j} \bar{a}_{i}} L_{\bar{a}_{k}}\right)+\left(R_{a_{j}} R_{a_{k} a_{i}}-R_{\bar{a}_{i} \bar{a}_{k}} R_{\bar{a}_{j}}\right) .
\end{aligned}
$$

Hence

$$
\begin{aligned}
x U_{i}= & 2\left(x \bar{a}_{i}\right) T \overline{a_{j} a_{k}}-2\left(x\left(a_{j} a_{k}\right)\right) T a_{i}+\left(a_{i} a_{j}\right)\left(a_{k} x\right)-\bar{a}_{k}\left(\left(\overline{a_{i} a_{j}}\right) x\right) \\
& +\left(x a_{j}\right)\left(a_{k} a_{i}\right)-\left(x\left(\overline{a_{k}} a_{i}\right)\right) \bar{a}_{j} \\
= & {\left[\bar{a}_{k} \bar{a}_{j}\left(\left(\bar{a}_{i} x\right) T\right)-\bar{a}_{k}\left(\left(\bar{a}_{j} \bar{a}_{i}\right) x\right)\right]+\left[\left(\left(x \bar{a}_{i}\right) T\right) \bar{a}_{k} \bar{a}_{j}\right.} \\
& \left.-\left(x\left(\bar{a}_{i} \bar{a}_{k}\right)\right) \bar{a}_{j}\right]+\left[\left(a_{i} a_{j}\right)\left(a_{k} x\right)-a_{i}\left(\left(a_{j} a_{k} x\right) T\right)\right] \\
& +\left[-\left(\left(x a_{j} a_{k}\right) T\right) a_{i}+\left(x a_{j}\right)\left(a_{k} a_{i}\right)\right] \\
= & \bar{a}_{k}\left[\bar{a}_{j}\left(\bar{a}_{i} x\right)+\bar{a}_{j}\left(\bar{x} a_{i}\right)-\left(\bar{a}_{j} \bar{a}_{i}\right) x\right] \\
& +\left[\left(x \bar{a}_{i}\right) \bar{a}_{k}+\left(a_{i} \bar{x}\right) \bar{a}_{k}-x\left(\bar{a}_{i} \bar{a}_{k}\right)\right] \bar{a}_{j} \\
& +\left[\left(a_{i} a_{j}\right)\left(a_{k} x\right)-a_{i}\left(a_{j}\left(a_{k} x\right)\right)-a_{i}\left(\left(\bar{x} \bar{a}_{k}\right) \bar{a}_{j}\right]\right. \\
& +\left[\left(x a_{j}\right)\left(a_{k} a_{i}\right)-\left(\left(x a_{j}\right) a_{k}\right) a_{i}-\left(\bar{a}_{k}\left(\bar{a}_{j} \bar{x}\right)\right) a_{i}\right] \\
= & -\bar{a}_{k} A\left(\bar{a}_{j}, \bar{a}_{i}, x\right)-\bar{a}_{k} A\left(\bar{a}_{j}, \bar{x}, a_{i}\right) \\
& +A\left(x, \bar{a}_{i}, \bar{a}_{k}\right) \bar{a}_{j}+A\left(a_{i}, \bar{x}, \bar{a}_{k i}\right) \bar{a}_{j} \\
& +A\left(a_{i}, a_{j}, a_{k} x\right)+A\left(a_{i}, \bar{x} \bar{a}_{k}, \bar{a}_{j}\right) \\
& -A\left(x a_{j}, a_{k}, a_{i}\right)-A\left(\bar{a}_{k}, \bar{a}_{j} \bar{x}, a_{i}\right) .
\end{aligned}
$$

The sum of the first two terms is 0 since $A(a, b, c)=A(a, \bar{b}, c)$ etc. The same reasoning applies to each of the succeeding pairs of terms. Hence $U_{i}=0$. To calculate $x V_{i}$ we note first that $d_{i}=\left(a_{k} \bar{a}_{k}-a_{j} \bar{a}_{j}\right) \bar{a}_{3}$ $-\left(\alpha_{j}-\alpha_{k}\right) a_{j} a_{k}$. Hence

$$
\begin{aligned}
V_{i}= & C R_{\left(a_{k} \bar{a}_{k}-a_{j} \bar{a}_{j}\right) \bar{a}_{i}-\left(\alpha_{j}-\alpha_{k}\right) C R_{a_{j} a_{k}}+\left(\alpha_{i}+\alpha_{j}\right) R_{\bar{a}_{j}} T R_{a_{k}}} \\
& +R_{\bar{a}_{j}} T R_{\bar{a}_{j} \bar{a}_{i}}-\left(\alpha_{k_{i}}+\alpha_{i}\right) R_{\bar{a}_{j}} T R_{a_{k}}-R_{a_{k} a_{i}} T R_{a_{k_{k}}} \\
& +\left(\alpha_{k}+\alpha_{i}\right) R_{a_{k}} L_{\bar{a}_{j}}+R_{a_{k}} L_{a_{k} a_{i}}-\left(\alpha_{i}+\alpha_{j}\right) R_{a_{k}} L_{\bar{a}_{j}} \\
& -R_{\bar{a}_{j} \bar{a}_{i} L_{\bar{a}_{j}}} \\
= & \left(\alpha_{k}-\alpha_{j}\right)\left(C R_{a_{j} a_{k}}-R_{\bar{a}_{j}} T R_{a_{k}}+R_{a_{k}} L_{\bar{a}_{j}}\right) \\
& +C R_{\left(a_{k} \bar{a}_{k}-a_{j} \bar{a}_{j}\right) \bar{a}_{i}}+R_{\bar{a}_{j}} T R_{\bar{a}_{j} \bar{a}_{i}}-R_{a_{i} a_{i}} T R_{a_{k}} \\
& +R a_{k} L_{a_{k} a_{i}}-R_{\bar{a}_{j} \bar{a}_{i}} L_{\bar{a}_{j}} .
\end{aligned}
$$

Since

$$
\begin{gathered}
x\left(C R_{a_{j} a_{k}}-R_{\bar{a}_{j}} T R_{a_{k}}+R_{a_{k}} L_{\bar{a}_{j}}\right)=\bar{x}\left(a_{j} a_{k}\right)-\left(\left(x \bar{a}_{\jmath}\right) T\right) a_{k} \\
\quad+\bar{a}_{j}\left(x a_{k}\right)=\bar{x}\left(a_{j} a_{k}\right)-\left(\left(\bar{a}_{j} x\right) T\right) a_{k}+\bar{a}_{j}\left(x a_{k}\right) \\
=-A\left(\bar{x}, a_{j}, a_{k}\right)-A\left(\bar{a}_{j}, x, a_{k}\right)=0
\end{gathered}
$$


and

$$
\begin{aligned}
& x\left(C R_{\left(a_{k} \bar{a}_{k}-a_{j} \bar{a}_{j}\right) \bar{a}_{i}}+R_{\bar{a}_{j}} T R_{\bar{a}_{j} \bar{a}_{i}}-R_{a_{k} a_{i}} T R_{a_{k}}\right. \\
& \left.+R_{a_{k}} L_{a_{k} a_{i}}-R_{\bar{a}_{j} \bar{a}_{i}} L_{\bar{a}_{j}}\right) \\
& =\left(a_{k} \bar{a}_{k}-a_{j} \bar{a}_{j}\right)\left(\bar{x} \bar{a}_{i}\right)+\left(a_{k} a_{i}\right)\left(x a_{k}\right)-\bar{a}_{j}\left(x\left(\bar{a}_{j} \bar{a}_{i}\right)\right) \\
& +\left(\left(x \bar{a}_{j}\right) T\right) \bar{a}_{j} \bar{a}_{i}-\left(\left(x\left(a_{k} a_{i}\right)\right) T\right) a_{k} . \\
& =\left(a_{k} \bar{a}_{k}-a_{j} \bar{a}_{j}\right)\left(\bar{x} \bar{a}_{i}\right)+a_{k}\left(\left(a_{i} x\right) a_{k}\right)-\left(\bar{a}_{j} x \bar{a}_{j}\right) \bar{a}_{i} \\
& +\left(\left(x \bar{a}_{j}\right) T\right) \bar{a}_{j} \bar{a}_{i}-\left(\left(x\left(a_{k} a_{i}\right)\right) T\right) a_{k} \\
& =a_{k}\left[\bar{a}_{k}\left(\overline{x_{i} a}\right)+\left(a_{i} x\right) a_{k}-\left(x\left(a_{k} a_{i}\right)\right) T\right] \\
& +\left[-\left(a_{j} \bar{a}_{j}\right) \bar{x}-\bar{a}_{j} x \bar{a}_{j}+\left(\left(x \bar{a}_{j}\right) T\right) \bar{a}_{j}\right] \bar{a}_{i} \\
& =0 \text {, }
\end{aligned}
$$

we have $V_{i}=0$. In a similar manner one proves that $W_{i}=0$. Hence $R_{a} R_{a^{2}}=R_{a^{2}} R a$ and $\mathfrak{S}$ is a Jordan algebra.

We shall show next that the algebras $\mathfrak{S}$ obtained from algebras $(D)$ which are not associative are not special. In fact, we have the following result.

Theorem 8.2. Let $\mathfrak{D}$ be an algebra (with an involution) which is not associative and let $\mathfrak{S}$ be the subalgebra of self-adjoint elements of $\mathfrak{U}_{J}$, $\mathfrak{A}=\mathfrak{D}_{n}, n \geqslant 3$ relative to a canonical involution in $\mathfrak{A}$. Then $\mathfrak{S}$ is not a special Jordan algebra. ${ }^{13)}$

Proof. Suppose $\mathfrak{S}$ is special and let $S$ be an isomorphism of $\mathfrak{H}$ into an algebra $\mathfrak{B}_{J}$ where $\mathfrak{B}$ is associative. Then it follows from Th. 7.2 that we may assume that $\mathfrak{B}=\mathfrak{F}_{n}$ where $\mathfrak{F}$ is associative and has an involution, that the image $\mathfrak{S}^{s}$ is the set of self-adjoint elements relative to a canonical involution in $\mathfrak{F}_{n}$ and that the images $u_{i j}^{S}$ of the $u_{i j}$ of $\mathfrak{F}$ generate the system of matrix units in $\mathfrak{F}_{n}$. Then $S^{-1}$ is an isomorphism of $\mathfrak{S}^{S}$ onto $\mathfrak{C}$ mapping the $u_{i j}^{S}$ into the elements $u_{i j}$ belonging to the nucleus of $\mathfrak{A}$. Hence, by Th. 7.3, the isomorphism $S^{-1}$ can be extended to a homomorphism of $\mathfrak{F}_{n}$ onto $\mathfrak{D}_{n}$. Then $\mathfrak{D}$ is a homomorphic image of an associative algebra, contrary to our assumption that $\mathfrak{D}$ is not associative.

\section{A structure theorem for Jordan algebras}

Let $\mathfrak{U}=\mathfrak{D}_{n}$ where $\mathfrak{D}$ has an involution and let $\mathfrak{S}$ be the set of self-adjoint elements relative to the standard involution in $\mathfrak{A}$. Then

13) This theorem contains Albert's result on the exceptional character of $M_{3}^{8}$ proved in [1] and [3]. 
$\left\{\right.$ contains the elements $e_{i i}$ and $u_{i j}=e_{i j}+e_{j i}=u_{j i}, i \neq j$. The nonzero Jordan relations (cf (35)) connecting these are

$$
\begin{aligned}
\left\{e_{i i} e_{i i}\right\} & =2 e_{i i}, \\
\left\{e_{i i} u_{i j}\right\} & =u_{i j}, \\
\left\{u_{i j} u_{j i}\right\} & =2\left(e_{i i}+e_{j j}\right), \\
\left\{u_{i j} u_{j k}\right\} & =u_{i k},
\end{aligned}
$$

if $i, j, k$ are $\neq$. Also we have the fact that $\frac{1}{2} \sum e_{i i}$ acts as the identity relative to \{\}. In this section we shall determine the structure of the Jordan algebras which possess sets of elements of this type. Our result will be an abstract analogue of Th. 7.2.

Let $\Im$ be a Jordan algebra with an identity element and assume that $1=\sum_{1}^{n} e_{i}$ where the $e_{i}$ are orthogonal idempotent elements. Let $\Im=\sum_{i \leq j} \oplus \widetilde{\Im}_{i j}$ be the Peirce decomposition relative to the $e_{i}$. Then we have the relations $(26)$ connecting the $\Im_{i j}$. Moreover, if $x_{i j}$, etc. denotes an element of $\Im_{i j}$ then we have the following

Lemma 1 . If $i, j, k, l$ are $\neq$, then

$$
\begin{gathered}
\left(x_{i i} y_{i j}\right) z_{j k}=x_{i i}\left(y_{i j} z_{j k}\right) \\
\left(x_{i j} y_{j j}\right) z_{j k}=x_{i j}\left(y_{j j} z_{j k}\right) \\
\left(x_{i j} y_{j k}\right) z_{k k}=x_{i j}\left(y_{j k} z_{k k}\right) \\
\left(x_{i j} y_{j k}\right) z_{j k}+\left(x_{i j} z_{j k}\right) y_{j k}=x_{i j}\left(y_{j k} z_{j k}\right) . \\
\left(x_{i j} y_{j k}\right) z_{k l}=x_{i j}\left(y_{j k} z_{k l}\right) .
\end{gathered}
$$

Proof. The proofs are obtained by substituting $a=e_{i}, b=x_{-}$, $c=y_{-}, d=z_{-}$in the basic Jordan identity

$$
a b c d+a d c b+a(b d c)=(a b)(c d)+(a c)(b d)+(a d)(b c) .
$$

We now suppose that $\Im$ is a Jordan algebra with identity element 1 containing $\frac{1}{2} n(n+1)$ elements $e_{i i}, u_{i j}=u_{j i}, i \neq j$, satisfying (52) (that is, $e_{i i}^{2}=2 e_{i i}, e_{i i} e_{j \jmath}=0$, etc). Assume, moreover, that $n \geqslant 3$ and $\frac{1}{2} \sum e_{i i}=1$. Then the elements $e_{i}=\frac{1}{2} e_{i i}$ are orthogonal idempotents with sum 1. Let $\mathfrak{\Im}=\sum_{i \leq j} \oplus \mathfrak{\Im}_{i j}$ be the Peirce decomposition relative to these elements. Put $U_{i j}=R_{u_{i j}}$.

LEMMA 2. $U_{i j}$ maps $\mathfrak{\Im}_{i i}+\mathfrak{\Im}_{i j}+\mathfrak{\Im}_{j j}$ into itself and satisfies $U_{i j}^{3}=4 U_{i j}$ in this space. $U_{i j}^{2}$ maps $\Im_{i j}$ into itself and $U_{i j}^{2}\left(U_{i j}^{2}-4\right)=0$ in $\mathfrak{\Im}_{i j} . \quad U_{i j}$ is a $1-1$ mapping of $\mathfrak{\Im}_{i i}$ into $\mathfrak{\Im}_{i j}$. 
Proof. The first parts of the first two statements follow from: $\Im_{i j}^{2} \subseteq \Im_{i i}+\mathfrak{\Im}_{j j}, \mathfrak{\Im}_{i j} \mathfrak{\Im}_{i i} \subseteq \Im_{i j}$. Write $u=u_{i j}, e=e_{i}+e_{j}=\frac{1}{2}\left(e_{i i}+e_{j j}\right)$. Then $u^{2}=4 e, e u=u$. Hence $u^{3}=4 u$ and $R_{u}^{3}=6 R_{e} R_{u}-2 R_{u}$, by (7). Since $R_{e}=1$ in $\mathfrak{\Im}_{i i}+\mathfrak{\Im}_{i j}+\mathfrak{\Im}_{j j}, R_{u}^{3}=4 R_{u}$ in this space. Hence $U_{i j}^{3}=4 U_{i j}$ here and $U_{i j}^{4}=4 U_{i j}^{2}$ in $\mathfrak{\Im}_{i j}$. To prove the last statement we substitute $a=x_{i i} \in \mathfrak{S}_{i i}, b=u_{i j}, c=u_{i j}, d=e_{i}$ in (53) to obtain $x_{i i} u_{i j} u_{i j} e_{i}=2 x_{i i}$.

For $x_{i j} \in \Im_{i j}$, define

$$
\bar{x}_{i j}=\frac{1}{2} x_{i j} U_{i j}^{2}-x_{i j} .
$$

Then, by Lemma $2, x_{i j} \rightarrow \bar{x}_{i j}$ is a linear transformation of period two in $\mathfrak{\Im}_{i j}$. Any $x_{i j}$ can be written in one and only one way in the form $y_{i j}+z_{i j}$ where $\bar{y}_{i j}=y_{i j}, \bar{z}_{i j}=-z_{i j}$. Also $\bar{y}_{i j}=y_{i j}$ if and only if $y_{i j} U_{i j}^{2}=4 y_{i j}$ and $\bar{z}_{i j}=-z_{i j}$ if and only if $z_{i j} U_{i j}^{2}=0$. Since $U_{i j}^{3}=4 U_{i j}$ in $\mathfrak{\Im}_{i i}+\mathfrak{I}_{i j}+\mathfrak{\Im}_{j j}, x U_{i j}^{2}=0$ in this space implies $x U_{i j}=0$. We now substitute $a=x_{i j}, \quad b=u_{i j}=c, d=y_{j k}$ in (53). Assuming $i, j, k \neq$, this gives

$$
\begin{gathered}
x_{i j} u_{i j} u_{i j} y_{j k}=-x_{i j} y_{j k} u_{i j} u_{i j}-x_{i j}\left(y_{j k} u_{i j} u_{i j}\right)+\left(x_{i j} y_{j k}\right) u_{i j}^{2} \\
+2\left(x_{i j} u_{i j}\right)\left(y_{j k} u_{i j}\right) .
\end{gathered}
$$

By Lemma 1, $\left(\left(x_{i j} y_{j k}\right) u_{i j}\right) u_{i j}=\frac{1}{2}\left(x_{i j} y_{j k}\right) u_{i j}^{2}$ and $\left(y_{j k} u_{i j}\right) u_{i j}=\frac{1}{2} y_{j k} u_{i j}^{2}$. Hence we obtain $x_{i j} u_{i j} u_{i j} y_{j k}=2\left(x_{i j} u_{i j}\right)\left(y_{j k} u_{i j}\right)$, or

$$
\left(x_{i j} U_{i j}^{2}\right) y_{j k}=2\left(x_{i j} U_{i j}\right)\left(y_{j k} U_{i j}\right), \quad i, j, k \neq .
$$

Similarly, if we substitute $a=x_{i j}, b=y_{j k}, c=u_{i k}=d, i, j, k \neq$, in the basic identity and again make use of Lemma 1, then we obtain $\left(x_{i j} y_{j k}\right) U_{i k}^{2}=2 x_{i j} y_{j k}+2\left(x_{i j} U_{i k}\right)\left(y_{j k} U_{i k}\right)$, or

$$
\overline{x_{i j} y_{j k}}=\left(x_{i j} U_{i k}\right)\left(y_{j k} U_{i k}\right), \quad i, j, k \neq .
$$

Finally, we shall need the following relation

$$
x_{i l} y_{i k}=\left(x_{i l} U_{i j}\right)\left(y_{i k} U_{i j}\right), \quad i, j, k, l \neq .
$$

This can be obtained by starting with $a=x_{i l}, b=u_{i j}=c, d=y_{i k}$.

LEMmA 3. $\quad \bar{x}_{i j}=x_{i j} U_{i k} U_{i j} U_{j k}=x_{i j} U_{j k} U_{i j} U_{i k}, \quad i, j, k \neq$.

Proof. $\quad x_{i j} U_{i k} U_{i j}=\left(u_{i k} x_{i j}\right) u_{i j}=-\left(u_{i k} u_{i j}\right) x_{i j}+u_{i k}\left(x_{i j} u_{i j}\right)=-x_{i j} U_{k j}$ $+x_{i j} U_{i j} U_{i k}=-x_{i j} U_{k j}+\left(x_{i j} U_{i j}\right)\left(u_{j k} U_{i j}\right)=-x_{i j} u_{j k}+\frac{1}{2}\left(x_{i j} U_{i j}^{2}\right) u_{j k}$, by (55). Hence $x_{i j} U_{i k} U_{i j}=\bar{x}_{i j} U_{j k}$. The first assertion now follows easily from Lemma 1. The second relation is just a re-statement of this. 
We now set $\mathfrak{D}=\mathfrak{\Im}_{12}$ and we make this vector space into an algebra by introducing a multiplication $x$ in $\mathscr{D}$ by

$$
x \times y=\left(x U_{23}\right)\left(y U_{13}\right) .
$$

Evidently, this result is in $\mathscr{D}$ and the function $x \times y$ is bilinear. It is clear also that $u_{12}=1$ in $\mathfrak{D}$.

Lemma 4. The conjugation $x \rightarrow \bar{x}$ is an involution in $\mathfrak{D}$.

Proof. If $x, y \in \mathfrak{D}=\mathfrak{\Im}_{12}$ then

$$
\overline{x \times y}=\overline{\left(x U_{23}\right)\left(y U_{13}\right)}=\left(x U_{23} U_{12}\right)\left(y U_{13} U_{12}\right),
$$

by (56). On the other hand, $x U_{23} U_{12}=x U_{23} U_{12} U_{13}^{2}=\bar{x} U_{13}$ by Lemmas 1 and 3 and similarly $y U_{13} U_{12}=\bar{y} U_{23}$. Hence $\left(x U_{23} U_{12}\right)\left(y U_{13} U_{12}\right)$ $=\left(\bar{y} U_{23}\right)\left(\bar{x} U_{13}\right)=\bar{y} \times \bar{x}$.

If $x \in \mathfrak{D}=\mathfrak{\Im}_{12}$ we define

$$
\begin{gathered}
x_{12}=x ; x_{1 j}=x U_{2 j}, \quad j>2 ; x_{j_{1}}=\bar{x}_{1 j}, \quad j \geqslant 2 \\
x_{i_{2}}=x U_{1 i}, \quad x_{2 i}=\bar{x}_{i_{2}}, \quad i>2 \\
x_{i j}=x U_{1 i} U_{2 \jmath}, \quad 1,2, i, j \neq .
\end{gathered}
$$

Note that if $1,2, i, j$ are $\neq$ then $\left(x u_{1 i}\right) u_{2 j}=\left(u_{i_{1}} x\right) u_{2 j}=u_{i_{1}}\left(x u_{2 j}\right)$. Hence $x_{i j}=x U_{2 j} U_{1 i}$ also. Note also that $u_{i j}=1_{i j}\left(1=u_{12}\right)$.

LEMma 5. $\quad \bar{x}_{i j}=x_{j i}$ for all $i, j \neq$.

Proof. This is true, by definition, if $i$ or $j$ is 1 or 2 . Hence suppose $1,2, i, j \neq$. Then $\quad \bar{x}_{i j}=\left(x U_{2 i} U_{12} U_{1 i}\right) U_{1 i} U_{2 j}=x U_{2 i} U_{12} U_{2 j}$ $=\left(x_{1 i} u_{12}\right) u_{2 j}=x_{1 i}\left(u_{12} u_{2 j}\right)=x_{1 i} u_{1 j}=x U_{i i} U_{1 j}=x_{j i}$.

By the definition of the multiplication in $\mathcal{D}$, we have $x_{13} y_{32}$ $=(x \times y)_{12}$. More generally, we have

LEmma 6 . If $i, j, k \neq$, then

$$
x_{i j} y_{j k}=(x \times y)_{i k} .
$$

Proof. By Lemma 5, if (60) holds for particular triple $(i, j, k)$, then it holds for $(k, j, i)$. Consider first $x_{12} y_{23}=\left(x_{13} U_{23}\right) y_{23}=-\left(x_{13} y_{23}\right)$ $U_{23}+x_{13}\left(y_{23} U_{23}\right)=-(x \times \bar{y})_{13}+\left(x_{12} U_{23}\right)\left(y_{23} U_{23}\right)=-(x \times \bar{y})_{13}+\frac{1}{2} x_{12}\left(y_{23} U_{23}^{2}\right)$. Thus $x_{12} \bar{y}_{23}=(x \times \bar{y})_{13}$ and $(60)$ holds for $(1,2,3)$. Note that $x_{21} U_{13}$ $=\bar{x}_{12} U_{13}=\bar{x}_{32}=x_{23}$. Hence $x_{21} y_{13}=\left(x_{23} U_{13}\right) y_{13}$ and the argument just used for $(1,2,3)$ shows that $(60)$ holds for $(2,1,3)$. Thus this relation holds for all permutations of $(1,2,3)$. By definition, $x_{12} U_{2 i}$ 
$=x_{1 i}, i>3$. Also $x_{13} U_{3 i}=x_{13}\left(u_{23} u_{2 i}\right)=\left(x_{13} u_{23}\right) u_{2 i}=x_{12} u_{2 i}=x_{1 i}, i>3$. Similarly, $x_{21} U_{1 i}=x_{2 i}=x_{23} U_{3 i}, x_{31} U_{1 i}=x_{3 i}=x_{32} U_{2 i}, i>3$. Now let $j>3, i, k \leqslant 3$. Choose $l \leqslant 3, l \neq i, k$. Then $x_{i j}=x_{i l} U_{l j}, y_{j k}=y_{k i} U_{l j}$. Hence, by (57), $x_{i j} y_{j k}=\left(x_{i l} U_{l j}\right)\left(y_{l k} U_{l j}\right)=x_{i l} y_{l k}=(x \times y)_{i k}$. Hence $(60)$ is valid for $i, k \leqslant 3, j$ arbitrary. Next let $i \leqslant 3, j, k$ arbitrary. Choose $l \leqslant 3, l \neq i, j$. Then $(60)$ holds for $(i, j, l)$. We may suppose also that $k>3$; hence $i, j, k, l$ are $\neq$. If $j \leqslant 3, y_{j k}=y_{j l} U_{l k}$ was proved before. If $j>3$ and $l=2, y_{j k}=y_{j l} U_{l n}$, by definition. If $j>3$ and $l=1$, then $y_{j l} U_{l k}=y_{j 1} U_{1 k}=y_{1 j} U_{1 k}=\left(\overline{y_{12} u_{2 j}}\right) u_{1 k}=\left(u_{1 k} y_{12}\right) u_{2 j}=y_{k j}=y_{j k}$. If $j>3$, $l=3, y_{j l} U_{l k}=y_{j 3} U_{3 k}=y_{j 2} U_{23} U_{3 k}=y_{j 2} U_{2 k}=y_{j k}$. Hence in all cases $y_{j k}=y_{j l} U_{l k}$. Then $x_{i j} y_{j k}=x_{i j}\left(y_{j l} U_{l k}\right)=\left(x_{i j} y_{j l}\right) U_{l k}=(x \times y)_{i l} U_{l k}=(x \times y)_{i k}$. This proves the formula for $i \leq 3, j, k$ arbitrary. A similar argument can be used to free the index $i$.

Set $E_{i}=\frac{1}{4} R_{e_{i i}}$ and define, for $x \in \mathfrak{D}$,

$$
x_{i i}=x_{i j} U_{j i} E_{i}, \quad i \neq j \text {. }
$$

If $i, j, k \quad$ are $\neq$ then $\quad x_{i k} u_{k i}=\left(x_{i j} u_{j k}\right)\left(u_{j k} u_{j i}\right)=-\frac{1}{2}\left(x_{i j} u_{j i}\right) u_{j k}^{2}$ $+\frac{1}{2}\left[x_{i j} u_{k j} u_{k j} u_{j i}+x_{i j} u_{j i} u_{k j} u_{k j}+x_{i j}\left(u_{j i} u_{k j} u_{k j}\right)\right]=-\left(x_{i j} u_{j i}\right) e_{j j}+x_{i j} u_{j i}$ $+\frac{1}{2} x_{i j} u_{j i} u_{k j} u_{k j}$. Hence $\left(x_{i k} u_{k i}\right) e_{i i}=\left(x_{i j} u_{j i}\right) e_{i i}$ and so $x_{i i}$ is independent of $j$.

LEMMA 7. If $i \neq j$, then

$$
x_{i i} y_{i j}=\frac{1}{2}((x+\bar{x}) \times y)_{i j} .
$$

Proof. Choose $k$ so that $i, j, k$ are $\neq$. Then $4 x_{i i} y_{i j}=x_{i k} u_{k i} e_{i i} y_{i j}$ $=-x_{i k} y_{i j} e_{i i} u_{k i}-x_{i k}\left(u_{k i} y_{i j} e_{i i}\right)+\left(x_{i k} e_{i i}\right)\left(y_{i j} u_{k i}\right)+\left(x_{i k} u_{k i}\right)\left(e_{i i} y_{i j}\right)+\left(x_{i k} y_{i j}\right)\left(e_{i i} u_{k i}\right)$ $=x_{i k} y_{k j}+x_{i k} u_{k i} y_{i j}+x_{i k} y_{i j} u_{k i}=(x \times y)_{i j}+x_{i k} u_{k i} y_{i j}+(\bar{x} \times y)_{i j}$. Since $x_{i k} u_{k i} y_{i j}$ $=\frac{1}{2} x_{i k} u_{k i} e_{i i} y_{i j}=2 x_{i i} y_{i j}$, this gives $(62)$.

LEMMA 8. If $i \neq j$, then

$$
\begin{gathered}
\left(x_{i j} y_{j i}\right) e_{i i}=4(x \times y)_{i i} \\
x_{i i} y_{i i}=\left\{\frac{1}{2}(x+\bar{x}), \frac{1}{2}(y+\bar{y})\right\}_{i}
\end{gathered}
$$

where $\{a b\} \equiv a \times b+b \times a$.

Proof. Choose $k \neq i, j$. Then $4 x_{i i} y_{i i}=\frac{1}{4}\left(x_{i j} u_{j i} e_{i i}\right)\left(y_{i k} u_{k i} e_{i i}\right)$ $=\left(x_{i j} u_{j i}\right)\left(y_{i k} u_{k i}\right)=-\left(x_{i j} y_{i k}\right)\left(u_{j i} u_{k i}\right)-\left(x_{i j} u_{k i}\right)\left(y_{i k} u_{j i}\right)+x_{i j} u_{j i} y_{i k} u_{k i}+x_{i j} u_{k i} y_{i k} u_{j i}$ $+x_{i j}\left(u_{j i} u_{k i} y_{i k}\right)=-(\bar{x} \times y)_{j k} u_{j k}-x_{k j} y_{j k}+2 x_{i i} y_{i k} u_{k i}+(y \times x)_{i j} u_{j i}+x_{i j} y_{i j}$. If we substitute $2 x_{i i} y_{i k} u_{k i}=((x+\bar{x}) \times y)_{i k} u_{k i}$ (Lemma 7$)$, we obtain 


$$
\begin{gathered}
4 x_{i i} y_{i i}=-(\bar{y} \times x)_{k j} u_{j k}-x_{k j} y_{j k}+((x+\bar{x}) \times y)_{i k} u_{k i} \\
+(y \times x)_{i j} u_{j i}+x_{i j} y_{i j} .
\end{gathered}
$$

Multiplication by $e_{k x}$ gives

$$
0=-4(\bar{y} \times x)_{k k}-\left(x_{k j} y_{j k}\right) e_{k k}+4(\bar{y} \times(x+\bar{x}))_{k i k} .
$$

Hence $\left(x_{k j} y_{j k}\right) e_{k k}=4(\bar{y} \times \bar{x})_{k k}$. Now multiply (65) by $e_{i i}$ to obtain

$$
\begin{aligned}
8 x_{i i} y_{i i} & =4((x+\bar{x}) \times y)_{i i}+4(y \times x)_{i i}+\left(x_{i j} \bar{y}_{j i}\right) e_{i i}, \\
2 x_{i i} y_{i i} & =((x+\bar{x}) \times y)_{i i}+(y \times x)_{i i}+(y \times \bar{x})_{i i} \\
& =\{(x+\bar{x}), y\}_{i i} .
\end{aligned}
$$

If we take $y=1$ we obtain $y_{i i} e_{i i}$ so that, by $(66), 2 x_{i i}=x_{i i}+\bar{x}_{i i}$. Thus $x_{i i}=\bar{x}_{i i}$. Hence, by $(66), 2 x_{i i} y_{i i}=\{x+\bar{x}, \bar{y}\}_{i i}$. If we average this with (66) we obtain (64). Also, our previous relation: $\left(x_{k j} y_{j k}\right) e_{k k}$ $=4(\bar{y} \times \bar{x})_{k i k}$ now gives (63).

We are now ready to prove the following result which will play a fundamental role in the sequel.

Theorem 9.1. Let $\Im$ be a Jordan algebra containing an identity 1 and elements $e_{i i}, u_{i j}=u_{j i}, i \neq j, i, j=1,2, \cdots, n$, such that

$$
\begin{gathered}
e_{i i}^{2}=2 e_{i i}, \quad e_{i i} u_{i j}=u_{i j}, \quad u_{i j} u_{j i}=2\left(e_{i i}+e_{j j}\right), \\
u_{i j} u_{j k}=u_{i k}, \quad i, j, k \neq
\end{gathered}
$$

and all other products are 0 . Assume, moreover, that $\frac{1}{2} \sum e_{i i}=1$ and $n \geqslant 3$. Then $\Im$ is isomorphic to the subalgebra of self-adjoint elements of a suitable $\mathfrak{U}_{J}$ where $\mathfrak{U}=\mathfrak{D}_{n}$ and the involution in $\mathfrak{A}$ is standard. Moreover, if $n>3$, then $\mathfrak{D}$ is associative and if $n=3$, $\mathfrak{D}$ is alternative and the self-adjoint elements of $\mathfrak{D}$ are in the nucleus.

Proof. Consider the Peirce decomposition $\mathfrak{\Im}=\sum \oplus \mathfrak{S}_{i j}$, relative to the set of orthogonal idempotents $e_{i}=\frac{1}{2} e_{i i}$. If $y \in \Im_{i j}, i \neq j$, then it is clear from (59) that $y=x_{i j}=\bar{x}_{i j}$ for a uniquely determined $x$ in $\mathfrak{D}=\mathfrak{\Im}_{12}$. By the proof of Lemma 2 and (61), any $y \in \Im_{i i}$ can be written as $x_{i i}, x \in \mathfrak{D}$, and we have seen that $x_{i i}=\bar{x}_{i i}$. Hence we can write $y$ as $x_{i i}$ where $\bar{x}=x$. It is easy to see (cf. (62)) that the selfadjoint element $x$ is uniquely determined by $y$. We now introduce the matrix algebra $\mathfrak{D}_{n}$ and denote matrix multiplication in it by $\times$. We denote the matrix units in $\mathfrak{D}_{n}$ by $E_{i j}, i, j=1,2, \cdots, n$. By the remarks just made there exists a $1-1$ linear mapping of $\Im$ into $\mathfrak{D}_{n}$ such that 


$$
\begin{array}{ll}
x_{i j} \rightarrow x E_{i j}+\bar{x} E_{j i}, & i \neq j \\
x_{i i} \rightarrow x E_{i i}, & \bar{x}=x .
\end{array}
$$

The formulas $(60),(62),(63)$ and (64) show that this is an isomorphism

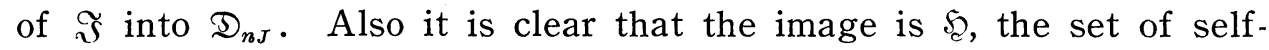
adjoint elements relative to the standard involution in $\mathfrak{U}$. If $n \geqslant 4$ then the last equation of Lemma 1 implies that $\mathfrak{D}$ is associative. If $n=3$, the first three equations of this lemma imply that if $\bar{x}=x$ then $x$ is in the nucleus. The fourth equation of Lemma 1 shows that

$$
(x \times y) \times \bar{y}=x \times(y \times \bar{y}), \quad(y \times \bar{y}) \times x=y \times(\bar{y} \times x) .
$$

Since $y \times \bar{y}$ is in the nucleus, these imply that $A(x, y, \bar{y})=0=A(y, \bar{y}, x)$. It is easy to see that these are sufficient conditions that $\mathfrak{D}$ is alternative. This completes the proof.

Remark. The hypotheses of the theorem can be modified somewhat as follows. Assume that we have elements $e_{i i}$ and $u_{1 j}, j=2, \cdots, n$ for which the non-zero products are $e_{i i}^{2}=2 e_{i i}, e_{11} u_{1 j}=u_{1 j}=u_{1 j} e_{j j}$, $u_{1 j}^{2}=2\left(e_{11}+e_{j j}\right)$. Then we can define $u_{i j}=u_{1 i} u_{1 j}$ for $1<i<j$ and $u_{k l}=u_{l k}$ for all $k \neq l$. Then the Jordan identities imply (67). Hence the conclusion of Theorem 9.1 holds (assuming $\frac{1}{2} \sum e_{i i}=1, n \geqslant 3$ ).

\section{Applications to representation theory : The Jordan case}

The structure results which we have obtained for the algebras $\mathscr{f}$ give considerable information on the module theory of such algebras. They can also be used to derive the main result on alternative bimodules for associative matrix algebras (Th. 11.1).

We suppose first that $\mathfrak{S}$ is the set of self-adjoint elements relative to a canonical involution in an associative algebra $\mathfrak{A}=\mathfrak{D}_{n}$. If $n \geqslant 3$ then, by Th. 7.3, any special representation $S$ of $\mathfrak{S}$ can be extended to a representation of the associative enveloping algebra $\mathfrak{A}$. It is easy to see that this is equivalent to the following result.

Theorem 10.1. If $\mathfrak{S}$ is the subalgebra of $\mathfrak{A}_{J}$ of self-adjoint elements in the associative matrix algebra $\mathfrak{A}=\mathfrak{D}_{n}$ relative to a canonical involution and $n \geqslant 3$, then $\mathfrak{A} \ddot{i}$ the universal associative algebra $\mathfrak{U}_{s}(\mathfrak{L})$ of $\mathfrak{S}_{\text {. }}$.

Suppose next that $\mathfrak{M}$ is a unital module for $\mathfrak{S}$ and let $\mathfrak{F}=\mathfrak{S} \oplus \mathfrak{M}$ be the corresponding split null extension. Then $[F$ has an identity and, if the involution is standard, 5 contains elements $e_{i i}, u_{i j}$ satisfying the conditions of Th. 9.1. Hence, if $n \geqslant 4$, then it follows from 
this theorem that $\sqrt{ }$ is special. Hence, by Th. $5.1, \mathfrak{M}$ is a submodule

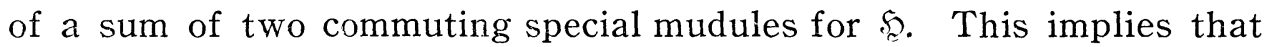
if $a \rightarrow R_{a}$ is a unital representation, then $a_{2}=a \otimes u+u \otimes a \rightarrow R_{a}$ determines a homomorphism of the associative algebra $\mathfrak{H}_{s}^{(2)}(\mathfrak{S})(\S 5)$ onto the enveloping associative algebra of the $R_{a}$. If $\mathfrak{U}_{1}(\mathfrak{S})$ denotes the universal associative algebra for the unital representations then the (right) regular representation of $\mathfrak{u}_{1}$ defines a unital representation of $\mathfrak{S}$. It follows that we have a homomorphism of $\mathfrak{U}_{s}^{(2)}(\mathfrak{S})$ onto $\mathfrak{U}_{1}(\mathfrak{S})$ mapping $a_{2}$ upon $a_{1}$. Since we always have a homomorphism of $\mathfrak{U}_{1}(\mathfrak{S})$ onto $\mathfrak{U}_{s}^{(2)}(\mathfrak{S})$ mapping $a_{1}$ on $a_{2}$ it is clear that both mappings are isomorphisms. This result can be stated in a slightly unprecise form as follows.

Theorem 10.2. Let $\mathfrak{S}$ be as in the preceding theorem. Assume the involution is standard and $n \geqslant 4$. Then the universal associative algebra $\mathfrak{u}_{1}(\mathfrak{S})$ of the unital representations coincides with the universal associative algebra $\mathfrak{U}_{s}^{(2)}(\mathfrak{S})$ of sums of commuting special representations.

This theorem focuses attention on the structure of the algebra $\mathfrak{u}_{s}^{(2)}(\mathfrak{S})$. Now, we observe first that any Kronecker square $\mathfrak{A} \otimes \mathfrak{A}$ possesses an automorphism $P: \sum x_{i} \otimes y_{i} \rightarrow \sum y_{i} \otimes x_{i}$ of period two. We shall call $P$ the exchange automorphism in $\mathfrak{U} \otimes \mathfrak{A}$. Evidently, the subalgebra $\mathfrak{B}$ of elements invariant under $P$ is the totality of elements of the form $\sum\left(x_{i} \otimes y_{i}+y_{i} \otimes x_{i}\right)$, that is, the subspace of symmetric tensors in the tensor space $\mathfrak{A} \otimes \mathfrak{A}$. Thus if $\mathfrak{U}$ is of finite dimensionality $n$, then $\operatorname{dim} \mathfrak{B}=n(n+1) / 2$. Now it is clear that the subalgebra $\mathfrak{U}_{s}^{(2)}\left(\mathfrak{S}_{\mathcal{E}}\right)$ of $\mathfrak{U}_{s} \otimes \mathfrak{U}_{s}$ generated by the elements $a_{s} \otimes u+u \otimes a_{s}$ is contained in the subalgebra of elements invariant under $P$. In a number of cases we can show that $\mathfrak{U}_{s}^{(2)}$ coincides with the subalgebra of elements invariant under $P$. One situation in which this is the case is given in the following theorem.

Theorem 10.3. Let $\mathfrak{S}$ be as in Th. 10.1, involution canonical, $n \geqslant 3$. Assume that the set $\mathfrak{S}$ of skew elements of $\mathfrak{U}$ coincides with [\$ㄹ] $]$. Then the associative algebra $\mathfrak{u}_{s}^{(2)}$ coincides with the subalgebra of $\mathfrak{u}_{s} \otimes \mathfrak{U}_{s}$ of elements invariant under the exchange automorphism $P$.

Proof. Set $A=a_{s} \otimes u+u \otimes a_{s}, B=b_{s} \otimes u+u \otimes b_{s}$ and form [AB] $=\left[a_{s} b_{s}\right] \otimes u \otimes\left[a_{s} b_{s}\right]$. By assumption if $q$ is any skew element of $\mathfrak{u}_{s}(\equiv \mathfrak{U})$ then $q$ is a sum of elements $\left[a_{s} b_{s}\right]$. Hence every element of the form $x \otimes u+u \otimes x, x$ in $\mathfrak{u}_{s}$, is in $\mathfrak{u}_{s}^{(2)}$. It follows that if $x$ and $y \in \mathfrak{U}_{s}$ then 


$$
x \otimes y+y \otimes x=(x \otimes u+u \otimes x)(y \otimes u+u \otimes y)-(x y \otimes u+u \otimes x y)
$$

is in $\mathfrak{U}_{s}^{(2)}$. Hence $\mathfrak{U}_{s}^{(2)}=\left\{\sum\left(x_{i} \otimes y_{i}+y_{i} \otimes x_{i}\right)\right\}$ and $\mathfrak{U}_{s}^{(2)}$ is the subalgebra of $\mathfrak{u}_{s} \otimes \mathfrak{u}_{s}$ of elements invariant under $P$.

Our results, particularly Th. 9.1, can also be used in another way to reduce the theory of unital $\mathfrak{S}$-modules to that of alternative (D-bimodules. In fact, this second method which we shall now consider is also applicable to the algebras $\mathfrak{S}$ which are exceptional. Thus we consider an algebra $\mathfrak{S}$ which is the set of self-adjoint elements of a matrix algebra $\mathfrak{U}=\mathfrak{D}_{n}, n \geqslant 3$, relative to a standard involution. Necessary (sufficient) conditions that $\mathfrak{C}$ is Jordan are $: n \geqslant 4$, $\mathfrak{D}$ associative; $n=3$, $\mathfrak{D}$ is either associative or its self-adjoint elements are in the nucleus (center). Let $\mathfrak{S}$ be Jordan and let $\mathfrak{M}$ be a unital $\mathfrak{S}$-module, $\mathfrak{F}=\mathfrak{S} \oplus \mathfrak{M}$ the split null extension. Then Th. 9.1 permits us to identify 5 with the set of self-adjoint elements of an algebra $\mathfrak{F}_{n}$ where $\mathfrak{F}$ is either associative or alternative. Since $\mathfrak{S}$ contains the $e_{i i}$ and $u_{i j}$, we may suppose that $\mathfrak{D}$ is a self-adjoint subalgebra of $\mathfrak{F}$ (Th. 7.1). Also, according to Th. 7.1, $\mathfrak{M}=\mathfrak{N}_{n \cap} \mathfrak{F}$ where $\mathfrak{N}$ is a selfadjoint ideal in $\mathfrak{F}$ such that $\mathfrak{N}^{2}=0$. Since $\mathfrak{S} \cap \mathfrak{M}=0, \mathfrak{N} \cap \mathfrak{D}=0$ and since $\mathfrak{F}=\mathfrak{T}+\mathfrak{M}, \mathfrak{F}=\mathfrak{D}+\mathfrak{R}$. Thus $\mathfrak{F}=\mathfrak{D} \oplus \mathfrak{N}$. Hence if $n \geqslant 4$, then $\Re$ is a unital associative bimodule and $\mathfrak{F}$ is the corresponding split null extension. If $n=3, \mathfrak{R}$ is unital alternative and $\mathfrak{F}$ is the corresponding split null extension. The notion of bimodule, however, does not give an adequate description of our situation; for, we must take into account also the involution in $\mathfrak{F}$ and in $\mathfrak{D}$. For this purpose we introduce the notion of a bimodule with involution.

Definition 10.1. Let $\mathfrak{D}$ be an algebra with an involution and let $D$ belong to a class $\Gamma$ of algebras satisfying a set of multilinear identities. Then a bimodule $\mathfrak{N}$ for $\mathfrak{D}$ in $\Gamma$ is said to be a bimodule with an involution if there is defined a linear transformation $x \rightarrow \bar{x}$ of period 2 of $\mathfrak{R}$ onto itself such that

$$
\overline{d x}=\bar{x} \bar{d}, \quad \overline{x d}=\bar{d} \bar{x}
$$

for all $d \in \mathfrak{D}, x \in \mathfrak{R}$. We call $x \rightarrow \bar{x}$ the involution of $\mathfrak{R}$.

If $\mathfrak{N}$ has this property then the involution in $\mathfrak{D}$ can be combined with that in $\mathfrak{N}$ to give an involution in the extension $\mathfrak{F}=\mathfrak{D} \oplus \mathfrak{R}$. Conversely, any involution in $\mathfrak{F}$ which extends that in $\mathfrak{D}$ can be used to turn the bimodule $\mathfrak{N}$ into a bimodule with an involution. In speaking of sub-bimodules of a bimodule with involution, etc. we shall mean sub-bimodules, etc. which are invariant relative to the involution $x \rightarrow \bar{x}$ given in the definition. Isomorphisms, homomorphisms, etc. for 
bimodules with involutions will mean mappings of this type which commute with the involutions.

Returning to the situation above we see that the Jordan $\$$-module $\mathfrak{M}$ determines a $\mathfrak{D}$-bimodule $\mathfrak{R}$ with an involution. If $n \geqslant 4, \mathfrak{R}$ is an associative bimodule and if $n=3, \mathfrak{R}$ is alternative, but even here we have the added condition that the self-adjoint elements of $\mathfrak{F}=\mathfrak{D} \oplus \mathfrak{R}$ are in the nucleus of $\mathfrak{F}$. If we define the nucleus (center) of a bimodule to be the intersection of the nucleus (center) of the null extension with the bimodule, then it is clear that the self-adjoint elements $(\bar{x}=x)$ of $\mathfrak{R}$ are contained in the nucleus. Also Th. 7.1 establishes a lattice isomorphism between the lattice of submodules of $\mathfrak{M}$ and the lattice of sub-bimodules of the $\mathfrak{D}$-bimodules with involution $\mathfrak{N}$. We therefore have the following theorem.

Theorem 10.4. Let $\mathfrak{S}$ be a Jordan algebra which is the subalgebra of self-adjoint elements of a matrix algebra $\mathfrak{A}=\mathfrak{D}_{n}, n \geqslant 3$, relative to a standard involution. Then any unital Jordan module $\mathfrak{M}$ for $\mathfrak{S}$ determines a unital alternative bimodule with involution $\mathfrak{N}$ for $\mathfrak{D}$ which is associative if $n>3$ and, in any case, has the property that the selfadjoint elements of the split null extension $\mathfrak{F}=\mathfrak{D} \oplus \mathfrak{N}$ are in the nucleus. Moreover, one has a lattice isomorphism between the lattice of submodules of $\mathfrak{M}$ and the lattice of sub-bimodules of the bimodule with involution $\mathfrak{R}$.

Our next result gives a reduction of the isomorphism problem from $\mathfrak{M}$ to $\mathfrak{R}$.

Theorem 10.5. Let $\mathfrak{S}$ be as in the preceding theorem and let $\mathfrak{M}_{1}$ and $\mathfrak{M}_{2}$ be unital $\mathfrak{S}_{-}$-modules, $\mathfrak{N}_{1}$ and $\mathfrak{N}_{2}$ the associated unital $\mathfrak{D}$-bimodules with involution given in the theorem. Then $\mathfrak{M}_{1}$ and $\mathfrak{M}_{2}$ are isomorphic if and only if $\mathfrak{R}_{1}$ and $\mathfrak{R}_{2}$ are isomorphic.

Proof. Let $\mathfrak{\xi}_{i}=\mathfrak{S} \oplus \mathfrak{M}_{i}, i=1,2$, be the split null extension of $\mathfrak{M}_{i}$, so that $\mathfrak{F}_{i}$ is the set of self-adjoint elements of $\mathfrak{F}_{i n}$ in where $\widetilde{F}_{i}=\mathfrak{D} \oplus \mathfrak{R}_{i}$ is the split null extension of $\mathfrak{N}_{i}$. We observe first that an isomorphism of $\mathfrak{M}_{1}$ onto $\mathfrak{M}_{2}$ determines an isomorphism $s$ of $\mathfrak{F}_{1}$ onto $\mathfrak{F}_{2}$ leaving the elements of $\mathfrak{S}$ invariant. By Th. 7.3 we can extend this isomorphism to a homomorphism $S$ of $\mathfrak{F}_{1 n}$ onto $\mathfrak{F}_{2 n}$. By using the inverse mapping we see that $S$ is an isomorphism. Moreovier, it leaves fixed the elements $u_{k j}, e_{k k}$ of $\mathfrak{k}$. Hence it leaves fixed the system of matrix units of $\mathfrak{F}_{1 n}$ (and of $\mathfrak{F}_{2 m}$ ). Hence $S$ induces an isomorphism of $\mathfrak{F}_{1}$ onto $\widetilde{F}_{2} \cdot{ }^{14)}$ Since our isomorphism of $\mathfrak{F}_{1}$ onto $\mathfrak{F}_{2}$

14) One needs to observe that $\mathfrak{F}_{i}$ is the subalgebra of $\mathfrak{F}_{i}$ of elements which commute with the matrix units. Cf. Prop. 7.1. 
is the identity on $\mathfrak{S}$, the extension is the identity on $\mathfrak{D}$. We note next that the isomorphism $s$ of $\mathfrak{F}_{1}$ onto $\mathfrak{F}_{2}$ maps $\mathfrak{M}_{1}$ onto $\mathfrak{M}_{2}$. It follows that its extension $S$ maps $\mathfrak{R}_{1 n}$ onto $\mathfrak{R}_{2 n}$ and, consequently, $S$ induces a linear transformation $\sigma$ of $\mathfrak{N}_{1}$ onto $\mathfrak{N}_{2}$. If $A \in \mathfrak{F}_{1 n}$ (or $\mathfrak{F}_{2 n}$ ) we let $A^{*}$ be its image under the standard involution in $\mathfrak{F}_{1 n}\left(\mathfrak{F}_{2 n}\right)$. Then if $A \in \mathfrak{F}_{1}, A^{*}=A$ and $\left(A^{s}\right)^{*}=A^{s}$ is in $\mathfrak{F}_{2}$. Hence $\left(A^{*}\right)^{s}=\left(A^{s}\right)^{*}$. Since $\mathfrak{F}_{1}$ generates $\mathfrak{F}_{1 n}$ this implies that $S$ commutes with the involutions in $\mathfrak{F}_{1 n}$ and $\mathfrak{F}_{2 n}$. Since the involutions in $\mathfrak{F}_{1}$ and $\mathfrak{F}_{2}$ are induced by these, $S$ commutes with the involutions in $\mathfrak{F}_{1}$ and $\mathfrak{F}_{2}$. Hence $\sigma$ is an isomorphism of the bimodule with involution $\mathfrak{N}_{1}$ onto $\mathfrak{N}_{2}$. The converse of all this is immediate.

The passage from $\mathfrak{M}$ to $\mathfrak{R}$ can be retraced. Thus suppose we have a unital alternative bimodule $\mathfrak{N}$ with split null extension $\mathfrak{F}=\mathfrak{D} \oplus \mathfrak{N}$. We assume that if $n>3$, $\mathfrak{D}$ and $\mathfrak{N}$ are associative and if $n=3$ then either this holds or the self-adjoint elements of $\mathfrak{F}$ are in the center. Then $\mathfrak{F}$ satisfies the conditions which insure that the algebra $\mathfrak{F}$ of self-adjoint elements of $\mathfrak{F}_{n}$ is Jordan. One sees readily that $\sqrt{5}$ has the form of a split null extension $\mathfrak{S} \oplus \mathfrak{M}$ and that the Jordan module $\mathfrak{M}$ determines the given $\mathfrak{N}$ as before. We remark also that, if $\mathfrak{F}$ is not associative, then $\mathbb{F}$ is not a special Jordan algebra and so $\mathfrak{M}$ can not be a submodule of a sum of two commuting special modules. This remark can be used to construct new exceptional Jordan algebras. ${ }^{15)}$

We observe finally that if $\mathfrak{D}$ is any associative algebra with identity and an involution, the theory of unital associative bimodules with involutions for $(D)$ can be reduced to that of right modules for a certain algebra (S). Consider first that Kronecker square $\mathscr{D} \otimes \mathfrak{D}$ and its natural automorphism $P$. We now form the cross product (S) of $\mathscr{D} \otimes(\mathbb{D}$ and this automorphism with factor set 1 . Thus the elements of (S) are representable uniquely in the form $u_{1}+u_{2} c$ where $u_{i} \in \mathfrak{D} \otimes \mathfrak{D}, c^{2}=1$ and $c u=u^{P} c, u \in \mathfrak{D} \otimes \mathfrak{D}$. Let $\mathfrak{i}$ be an associative unital $\mathfrak{D}$-bimodule with an involution. Then we define

$$
n\left(d_{1} \otimes d_{2}\right)=\overline{d_{1}} n d_{2}, \quad n c=\bar{n}
$$

and extend these to define a composition of $\mathfrak{R}$ and $\mathbb{S}$ into $\mathfrak{R}$. One verifies that $\mathfrak{N}$ is a right $(\mathfrak{S}$-module. It is easy to see that the theory of this $\mathbb{S}$-module is equivalent to that of the given $\mathfrak{D}$-bimodule with involution.

\section{Application to representation theory: The alternative case}

We consider now an associative matrix algebra $\mathfrak{D}_{n}$ (not necessarily

15) See $\$ 19$. 
involutorial) and we wish to study the alternative unital bimodules for $\mathfrak{D}_{n}$. Now if $\mathfrak{U}$ is any alternative algebra containing $n^{2}$ elements $e_{i j}$ such that $e_{i j} e_{k l}=\delta_{j k} e_{i l}, \sum e_{i i}=1$, one can deduce from results of Zorn [37] that, if $n \geqslant 3, \mathfrak{A}$ is associative. This can be used to show (via split null extensions) that every alternative unital bimodule for $\mathfrak{D}_{n}, n \geqslant 3$, is associative. However, the known results give little information for the case $n=2$. We shall therefore not attempt to adapt them to our purposes here. Instead, we shall derive the theory of alternative representations by another method which is based on the Jordan results previously obtained.

Our starting point is the observation that if $a \rightarrow L_{a}, a \rightarrow R_{a}$ is a birepresentation in $\mathfrak{M}$, then these mappings are special representations of $\mathfrak{A}_{J}, \mathfrak{U}=\mathfrak{D}_{n}(\mathrm{cf} .(16))$. Let $L(\mathfrak{A})$ denote the enveloping associative algebra of the $L_{a}$. Then by Th. 7.4, $L(\mathfrak{A})=L^{(1)}(\mathfrak{U}) \oplus L^{(2)}(\mathfrak{U}), L^{(i)}(\mathfrak{U})$ ideals, such that if $L_{a}^{(i)}$ denotes the component of $L_{a}$ in $L^{(i)}(\mathfrak{A}), a \rightarrow L_{a}^{(1)}$ is an associative homomorphism of $\mathfrak{A}$ and $a \rightarrow L_{a}^{(2)}$ is an associative anti-homomorphism. If the bimodule is unital, then the algebra decomposition $L(\mathfrak{U})=L^{(1)}(\mathfrak{U}) \oplus L^{(2)}(\mathfrak{U})$ gives a decomposition of $\mathfrak{M}$ as $\mathfrak{M}^{(1)} \oplus \mathfrak{M}^{(2)}$ where $\mathfrak{M}^{(i)}$ is the subspace $\mathfrak{M} L^{(i)}(\mathfrak{U})$. Evidently, $\mathfrak{M}^{(i)}$ is invariant relative to $L(\mathfrak{U})$ and the contraction of $L_{a}$ to $\mathfrak{M}^{(1)}$ is an associative homomorphism while the contraction to $\mathfrak{M}^{(2)}$ is an associative anti-homomorphism. We refer next to equation (9). According to this, for any $a \in \mathfrak{A}$, the derivation $X \rightarrow\left[X R_{a}\right]$ in the algebra of linear transformations of $\mathfrak{M}$ maps $L(\mathfrak{U})$ into itself. Since $\left(L^{(i)}(\mathfrak{U})\right)^{2}=L^{(i)}(\mathfrak{A})$, it follows that the ideals $L^{(i)}(\mathfrak{A})$ are invariant under the derivation. This in turn implies that the $\mathfrak{M}^{(i)}$ are invariant relative to the $R_{a}$. Hence the $\mathfrak{M}^{(i)}$ are sub-bimodules. In a similar manner we can decompose each $\mathfrak{M}^{(i)}$ into two sub-bimodules such that $a \rightarrow R_{a}$ is an associative homomorphism in one of these and an anti-homomorphism in the other. Now we have seen that $a \rightarrow R_{a}$ is a homomorphism if and only if $a \rightarrow L_{a}$ is an anti-homomorphism and if these conditions hold, then the module is associative. We therefore see that two of the four sub-bimodules which we considered are 0 . The bimodule $M$ is a direct sum of an associative one and a bimodule in which $a \rightarrow R_{a}$ is an associative anti-homomorphism and $a \rightarrow L_{a}$ is an associative homomorphism.

Bimodules of the last type do exist. Thus let $\mathfrak{A}=\Phi_{2}$ and let $a \rightarrow U_{a}$ be a representation of the associative algebra $\mathfrak{A}$. Also define $\vec{a}=\operatorname{tr}(a) 1-a$. Then it is known that $a \rightarrow \bar{x}$ is an involution in $\mathfrak{A}$. Now set

$$
L_{a}=U_{a}, \quad R_{a}=U_{\bar{a}}
$$


Then one can verify that $a \rightarrow L_{a}, a \rightarrow R_{a}$ is an alternative birepresentation of $\Phi_{2}$. We shall call such a birepresentation of $\Phi_{2}$ a Cayley birepresentation. The corresponding bimodules will be'called Cayley bimodules.

We can now prove the following theorem.

Theorem 11.1. Let $(\mathcal{D}$ be an associative algebra with an identity. Then any unital alternative bimodule for $\mathfrak{D}_{n}, n \geqslant 3$, is associative. Any unital alternative bimodule for $\Phi_{2}$ is a direct sum of an associative bimodule and a Cayley bimodule.

Proof. We have the decomposition of any alternative unital bimodule for $\mathfrak{A}=\mathfrak{D}_{n}, n \geqslant 2$, into an associative one $\mathfrak{M}_{1}$ and bimodule $\mathfrak{M}_{2}$ in which $a \rightarrow L_{a}$ is an associative homomorphism and $a \rightarrow R_{a}$ is an associative anti-homomorphism. Thus $L_{a b}=L_{a} L_{b}$ and $R_{a b}=R_{b} R_{a}$ holds in $\mathfrak{M}_{2}$. Hence, by (9)

$$
\left[L_{a} R_{b}\right]=\left[R_{b} R_{a}\right]=\left[L_{b} L_{a}\right]=\left[R_{a} L_{b}\right] .
$$

This implies that $T_{a} \equiv L_{a}+R_{a}$ commutes with all the $L_{b}, R_{b}$. Now, we have noted that $a \rightarrow T_{a}$ is a representation of $\mathfrak{U}_{J}(\operatorname{cf} \S 3$ after $(8))$. $T_{A(b, c, a)}=\left[\left[T_{a} T_{b}\right] T_{c}\right]=0($ eq $(16))$. Since $A(b, c, a)=\{\{b c\} a\}-\{b\{c a\}\}$ $=[[a b] c], T_{u}=0$ for every $u=[[a b] c]$ in $\mathfrak{A}$. If $\left\{e_{i j} ; i, j=1,2, \cdots, n\right\}$ is a system of matrix units for $\mathfrak{A}=\mathfrak{D}_{n}$ then $e_{i i}-e_{n n}=\left[\left[e_{i n} e_{n n}\right] e_{n i}\right]$ for $i \neq n$. Hence $T_{e_{i i}}=T_{e_{n n}}$. Since $\mathfrak{M}_{2}$ is a unital bimodule for I, $L_{1}=R_{1}=1$; hence $T_{1}=2$. It follows that $n T_{e_{n n}}=T_{1}=2$. On the other hand, since $\left\{e_{n n} e_{n n}\right\}=2 e_{n n}, T_{e_{n n}}$ satisfies the equation $x(x-1)(x-2)=0$ (cf the paragraph of eq $(22)$ ). These conditions are compatible only if either $\mathfrak{M i}_{2}=0$ or $n=2$. This proves the first statement in the theorem. Assume now that $n=2$ and $\mathfrak{D}=\Phi$. In this case, $T_{e_{11}}=1=T_{e_{22}}$. Also since $e_{12}=\left[\left[e_{12} e_{22}\right] e_{22}\right], T_{e_{12}}=0$ and similarly $T_{e_{21}}=0$. It follows that $T_{a}$ is the trace of a (multiplied by the identity mapping in $\mathfrak{M}_{2}$ ). Then $R_{a}=T_{a}-L_{a}=L_{\bar{c}}$ where $\bar{a}=\operatorname{tr} a-a$. Thus $\mathfrak{M}_{2}$ is a Cayley bimodule.

\section{Extension of the base field}

Let $\mathfrak{A}$ be an algebra belonging to a class $\Gamma$ of algebras satisfying multilinear identities. The identities in question are in a free nonassociative algebra $\mathfrak{S}(\mathfrak{B})$ based on a vector space $\mathfrak{B}$ over $\Phi$. Let $\mathrm{P}$ be an extension of the base field $\Phi$. Then we can form the vector space $\mathfrak{B}_{\mathrm{P}}$ and the algebras $\mathfrak{S}_{\mathrm{P}}$ and $\mathfrak{U}_{\mathrm{p}}$ obtained by extending the base field to $\mathrm{P}$. Since the identities defining $\Gamma$ are multilinear, it is 
clear that $\mathfrak{A}_{\mathrm{P}}$ satisfies these identities (as members of $\mathfrak{\subseteq}_{\mathrm{P}}$ ). This is another one of the main advantages of dealing with multilinear identities. In particular, it is clear that if $\mathfrak{A}$ is associative, Lie, alternative or Jordan then $\mathfrak{A}_{\mathrm{P}}$ is of the same type. Now let $a \rightarrow R_{a}, a \rightarrow L_{a}$ be birepresentation of $\mathfrak{A}$ acting in the bimodule $\mathfrak{M}$. Then if $\mathfrak{M}_{\mathbf{P}}$ is the space obtained by extending the base field of $\mathfrak{M}$ to $P$, it is immediate that $\mathfrak{M}_{\mathbf{P}}$ can be considered as an $\mathfrak{A}_{\mathrm{P}}$-bimodule. The corresponding birepresentation of $\mathfrak{A}_{\mathbf{P}}$ will be referred to as the one obtained from $(R, L)$ by extending the base field. In the alternative and Jordan cases, it is easy to see also that the various constructions of universal associative algebras commute with the extension of the base field. Thus if $\mathfrak{U}$ is a Jordan algebra and $\mathfrak{u}_{\boldsymbol{s}}(\mathfrak{U}), \mathfrak{u}(\mathfrak{U}), \mathfrak{U}_{1}(\mathfrak{A})$ are, respectively, the special universal associative algebra, the universal associative algebra of the special representations and the universal associative algebra of the unital representations of $\mathfrak{A}$, then

$$
\mathfrak{U}_{s}\left(\mathfrak{A}_{\mathbf{P}}\right)=\mathfrak{U}_{s}(\mathfrak{U})_{\mathbf{P}}, \quad \mathfrak{u}\left(\mathfrak{A}_{\mathbf{P}}\right)=\mathfrak{U}(\mathfrak{A})_{\mathbf{P}}, \quad \mathfrak{U}_{1}\left(\mathfrak{A}_{\mathbf{P}}\right)=\mathfrak{U}_{1}(\mathfrak{U})_{\mathbf{P}} .
$$

These remarks enable us to extend the foregoing results to a somewhat wider class of algebras. Thus in the Jordan case we do not need to assume that the given algebra is the set of self-adjoint elements of a matrix algebra, $n \geqslant 3$, relative to a canonical involution. It suffices to assume that the algebra obtained by making a suitable extension of the base field has this structure. A similar remark applies in the alternative case. Here our main theorem on birepresentations applies to any associative algebra $\mathfrak{A}$ for which there exists an extension $\mathrm{P}$ of the base field such that $\mathfrak{A}_{\mathrm{P}}=\mathfrak{D}_{n}, n \geqslant 2$. We shall not attempt to list sufficient conditions on $\mathfrak{A}$ which imply this property. We recall only that if $\mathfrak{A}$ is any finite dimensional central simple algebra properly containing the base field, then there exist splitting fields $\mathrm{P}$ such that $\mathfrak{A}_{\mathrm{P}}=\mathrm{P}_{n}$. We investigate now more closely the situation in the Jordan case.

First, let $\mathfrak{A}$ be an associative algebra with an involution and let $\mathfrak{S}(\mathfrak{A})$ denote the subalgebra of $\mathfrak{A}_{\boldsymbol{J}}$ of self-adjoint elements. If $\mathrm{P}$ is an extension of the base field then the given involution can be extended in one and only one way to an involution in $\mathfrak{A}_{\mathbf{P}}$ and $\mathfrak{S}_{\mathfrak{C}}\left(\mathfrak{A}_{\mathbf{P}}\right)$ $=\mathfrak{S}(\mathfrak{U})_{\mathrm{P}}$. Suppose now that $\mathfrak{A}_{\mathrm{P}}=\mathfrak{D}_{n}, n \geqslant 3$, and the extended involution is canonical (standard). In this case we shall say that the involution in $\mathfrak{A}$ is extension canonical (standard). We shall now show that the class of Jordan algebras to which our results apply is precisely the class of algebras $\mathfrak{S}(\mathfrak{U})$ where $\mathfrak{A}$ has an involution which is extension canonical. 
Theorem 12.1. Let $\mathfrak{\Im}$ be a Jordan algebra over a field $\Phi$. Then a necessary and sufflcient condition that there exists an extension field $\mathrm{P}$ of $\Phi$ such that $\mathfrak{\Im}_{\mathrm{P}}$ is isomorphic to the subalgebra of self-adjoint elements of an associative matrix algebra $\mathfrak{D}_{n}, n \geqslant 3$, with a canonical involution is that $\mathfrak{\Im}$ itself is isomorphic to the subalgebra of self-adjoint elements of an associative algebra $\mathfrak{A}$ with an involution which is extension canonical. ${ }^{16)}$

Proof. We may as well suppose that $\mathfrak{\Im}_{\mathrm{P}}=\mathfrak{S}_{\mathfrak{C}}\left(\mathfrak{D}_{n}\right)$ where $n \geqslant 3$, $\mathfrak{D}$ an associative algebra over $\mathrm{P}$, involution in $\mathfrak{D}_{n}$ canonical. Then if $\mathfrak{D}_{n}$ is considered as an algebra over $\Phi, \mathfrak{\Im}$ is a subalgebra of $\mathfrak{D}_{n J}$. Let $R$ be a 1-1 unital representation of the associative algebra $\mathfrak{D}_{n}$ by linear transformations in a vector space $\mathfrak{M}$ over $P$. We can now consider $\mathfrak{M}$ as a vector space over $\Phi, \mathfrak{D}_{n}$ as an algebra over $\Phi$. Then $R$ defines a representation of $\mathfrak{D}_{n}$ over $\Phi$ in $\mathfrak{M}$ over $\Phi$. If we form $(\mathfrak{M} \text { over } \Phi)_{\mathrm{P}}$ we can extend $R$ to a representation $R^{(1)}$ of $\mathfrak{D}_{n \mathrm{P}}$ in $(\mathfrak{M}$ over $\Phi)_{\mathrm{P}}$. Next we observe that $R$ induces a special representation of $\mathfrak{\Im}$ by linear transformations in $\mathfrak{M}$ over $\Phi$. This determines a special representation of $\mathfrak{\Im}_{P}$ in $(\mathfrak{M} \text { over } \Phi)_{P}$. By Th. 7.3 this can be extended to a representation $R^{(2)}$ of the associative algebra $\mathfrak{D}_{n}$ by linear transformations in $(\mathfrak{M} \text { over } \Phi)_{\mathbf{P}}$. Now let

$$
\mathfrak{U}=\left\{a \mid a \in \mathfrak{D}_{n}, R_{a}^{(1)}=R_{a}^{(2)}\right\} .
$$

Since $R^{(1)}$ is a linear mapping of $\mathfrak{D}_{n}$ over $\Phi, \mathfrak{X}$ is a subalgebra of $\mathfrak{D}_{n}$ over $\Phi$. If $j \in \mathfrak{F}, R_{j}^{(1)}=R_{j}^{(2)}$ so that $\mathfrak{\Im} \subseteq \mathfrak{A}$. Since the enveloping associative algebra of $\mathfrak{\Im}_{\mathbf{P}}$ is $\mathfrak{D}_{n}$ we can find a set of elements $\left\{a_{\boldsymbol{\alpha}}\right\}$ which are products of elements in $\mathfrak{\Im}$ and which form a basis for $\mathfrak{D}_{n}$ over P. Evidently the $a_{a} \in \mathfrak{Y}$. Now suppose that $\sum \rho_{i} a_{i}, \rho_{i} \in \mathrm{P}$, is in भ. Since $R_{\rho}^{(2)}=\rho$ for $\rho$ in $\mathrm{P}$ and $R_{a_{i}}^{(1)}=R_{a_{i}}^{(2)}$, our condition gives $\sum\left(R_{\rho_{i}}^{(1)}-\rho_{i}\right) R_{a_{i}}^{(1)}=0$. We assert that this implies that the $\rho_{i}$ are in $\Phi$. Thus we know that if $A_{1}, A_{2}, \cdots, A_{m}$ are linear transformations in $M$ over $\Phi$ which are $\Phi$-independent then their extensions in $(\mathfrak{M} \text { over } \Phi)_{\mathrm{P}}$ are $\mathbf{P}$-independent. Now express the $\rho_{i}=\sum \alpha_{i j} \lambda_{j}, \alpha_{i j} \in \Phi, \quad \lambda_{1}=1$, $\lambda_{j} \in \mathrm{P}$ and $\Phi$-independent. Then the elements $\lambda_{j} a_{i}$ are $\Phi$-independent; hence the linear transformations $R_{\lambda_{j}} R_{a_{i}}$ are $\Phi$-independent. It follows that the extensions $R_{\lambda_{j}}^{(1)} R_{a_{i}}^{(1)}$ are $\mathrm{P}$-independent. We have the relation

$$
\sum_{i}\left(\alpha_{i_{1}}-\rho_{i}\right) R_{a_{i}}^{(1)}+\sum_{i} \sum_{j 乙^{2}} \alpha_{i j} R_{\lambda_{j}}^{(1)} R_{a_{i}}^{(1)}=0 .
$$

Hence $\rho_{i}=\alpha_{i_{1}} \in \Phi$. This proves that $\mathfrak{U}$ is the $\Phi$-space spanned by

16) This result is a generalization of Theorems 3 and 4 of [15]. 
the $a_{a}$. Hence $\mathfrak{A}_{\mathrm{p}}=\mathfrak{S}_{n}$. Since the elements of $\Im$ are self-adjoint, the involution in $\mathfrak{D}_{n}$ induces an involution in $\mathfrak{A}$. Since $\mathfrak{\Im}_{\mathrm{P}}$ is the set of self-adjoint elements of the involution in $\mathfrak{D}_{n}, \mathfrak{\Im}$ is this set for $\mathfrak{A}$. This completes the proof.

\section{Jordan Algebras Defined by Symmetric Scalar Products}

\section{Definition of the algebras}

In this part we shall consider the representation theory of a second important class of Jordan algebras, namely, the algebras defined by symmetric scalar products. We begin with a finite dimensional vector space $\Im_{0}$ over $\Phi$ and a symmetric scalar product $(x, y)$ in $\Im_{0}$. Thus $(x, y)$ is a symmetric bilinear form defined on $\Im_{0}$ and having values in the base field $\Phi$. We form the vector space $\mathfrak{\Im}=\mathfrak{\Im}_{0} \oplus \Phi 1$ where $\Phi 1$ is the one dimensional space of multiples of 1 and we define a multiplication in $\mathfrak{\Im}$ by demanding that 1 is the identity and

$$
x y=(x, y) 1 \text {. }
$$

One verifies that $\mathfrak{\Im}$ is a Jordan algebra. We shall call $\Im$ the Jordan algebra of the symmetric scalar product $(x, y)$.

We recall that the radical $\Re$ of a symmetric scalar product $(x, y)$ in $\Im_{0}$ is the set of elements $z$ such that $(x, z)=0$ for all $x$ in $\mathfrak{\Im}_{0}$. $(x, y)$ is non-degenerate if $\Re=0$. The scalar product in $\mathfrak{\Im}_{0}$ is equivalent to $\left\langle x^{\prime}, y^{\prime}\right\rangle$ in $\Im_{\Im_{0}^{\prime}}^{\prime}$ if and only if there exists a 1-1 linear transformation $x \rightarrow x^{\prime}$ of $\Im_{0}$ onto $\Im_{0}^{\prime}$ such that $(x, y)=\left\langle x^{\prime}, y^{\prime}\right\rangle$ for all $x, y \in \mathfrak{\Im}_{0}$. If $\Re$ is the radical of $(x, y)$ then $(x, y)$ defines in an obvious way a non-degenerate symmetric scalar product in $\bar{\Im}=\mathfrak{\Im}_{0} / \mathfrak{R}$. If $\mathfrak{B}_{0}$ is a complement of $\Re=\mathfrak{\Im}_{0}\left(\mathfrak{\Im}_{0}=\mathfrak{B}_{0} \oplus \Re\right)$ then the contraction of $(x, y)$ to $\mathfrak{B}_{0}$ is equivalent to the scalar product induced by $(x, y)$ in $\mathfrak{\Im}_{0} / \mathfrak{R}$. It follows that $(x, y)$ is equivalent to $\left\langle x^{\prime}, y^{\prime}\right\rangle$ if and only if their radicals have the same dimensionality and the induced non-degenerate scalar products in the factor spaces modulo the radical are equivalent. An element $\beta \in \Phi$ is said to be represented by $(x, y)$ if there exists a non-zero $x$ in $\Im_{0}$ such that $(x, x)=\beta$.

PROPOSITION 13.1. (1) The radical $\mathfrak{R}$ of $(x, y)$ is an ideal in the Jordan algebra $\Im$ of $(x, y)$ such that $\mathfrak{R}^{2}=0$. (2) $\Re$ contains every ideal $\mathfrak{B}$ of $\Im$ such that $\mathfrak{B}^{2}=0$. (3) $\Im / \Re$ is the Jordan algebra of the induced non-degenerate scalar product in $\mathfrak{\Im}_{0} / \mathfrak{R}$. (4) If $(x, y)$ is nondegenerate and of $\operatorname{rank}>1$ then $\Im$ is simple. If $(x, y)$ is non- 
degenerate and of rank 1 then $\Im$ is either simple or a direct sum of two simple algebras according as $(x, y)$ does not or does represent 1 .

Proof. (1) and (3) are immediate. To prove (2) we note that if $\beta+u, u \in \mathfrak{F}_{0}$, has square $=0$ then $\beta=0$. Hence if $u v=(u, v)$ has zero square for all $v \in \mathfrak{\Im}_{0}$, then $u \in \Re$. This proves $(2)$. Now let $(x, y)$ be non-degenerate and $\operatorname{dim} \mathfrak{\Im}_{0}>1$. Let $\mathfrak{B}$ be a non-zero ideal in $\mathfrak{\Im}, \beta+u \neq 0$ an element of $\mathfrak{B}$. If $\beta=0, u \neq 0$ and we can choose $v \in \mathfrak{I}_{0}$ so that $(u, v) \neq 0$. This implies that $1 \in \mathfrak{B}$. If $\beta \neq 0$ we choose $v \neq 0$ so that $(u, v)=0$. (This is possible since $\operatorname{dim} \dddot{y}_{0}>1$.) Then $v=\beta^{-1}(\beta+u) v \in \mathfrak{B}$ and $\mathfrak{B}=\mathfrak{Y}$ as in the previous case. Hence $\mathfrak{I}$ is simple. Finally, let $(x, y)$ be non-degenerate and $\operatorname{dim} \mathfrak{\Im}_{0}=1$. Let $\mathfrak{B}$ be a proper ideal $\neq 0$ in $\Im$. Then, the argument just used shows that $\mathfrak{B}$ is the one-dimensional space of multiplies of $u+1, u \neq 0$ in $\mathfrak{\Im}_{0}$. Then $u(u+1)=u+(u, u) \in \mathfrak{B}$. Hence $(u, u)=1$. The converse is immediate. If $(u, u)=1$ then $e=\frac{1}{2}(u+1)$ satisfies $e^{2}=e$ and this implies that $\mathfrak{F}$ is direct sum of two simple one dimensional algebras.

PROPOSITION 13.2. The Jordan algebras $\Im$ and $\mathfrak{\Im}^{\prime}$ of $(x, y)$ and $\left\langle x^{\prime}, y^{\prime}\right\rangle$, respectively, are isomorphic if and only if $(x, y)$ and $\left\langle x^{\prime}, y^{\prime}\right\rangle$ are equivalent. If $(x, y)$ is non-degenerate then the group of automorphisms of $\mathfrak{F}$ is isomorphic to the orthogonal group of $(x, y)$.

Proof. If $(x, y)$ and $\left\langle x^{\prime}, y^{\prime}\right\rangle$ are equivalent then it is clear that $\mathfrak{\Im}$ and $\mathfrak{\Im}^{\prime}$ are isomorphic. Conversely assume $\mathfrak{\Im}$ and $\mathfrak{\Im}^{\prime}$ isomorphic. Then it is clear from Prop. $13.1(1)$ and $(2)$ that the radicals $\Re$ and $\Re^{\prime}$ have the same dimensionalities. Also, in view of $(3)$, it suffices to assume that $(x, y)$ and $\left\langle x^{\prime}, y^{\prime}\right\rangle$ are non-degenerate. In this case the non-zero elements of $\mathfrak{\Im}_{0}$ can be characterized by the two conditions: $u \neq \beta, u^{2}=\beta$. It follows that an isomorphism of $\mathfrak{\Im}$ onto $\mathfrak{J}^{\prime}$ maps $\mathfrak{\Im}_{0}$ onto the corresponding subspace $\mathfrak{\Im}_{0}{ }^{\prime}$. It therefore determines an equivalence of $(x, y)$ and $\left\langle x^{\prime}, y^{\prime}\right\rangle$. This proves the first statement of the proposition. The second one is now clear, too.

We assume now that $(x, y)$ is non-degenerate. Then we can extend this scalar product to a non-degenerate symmetric scalar product in $\mathfrak{\Im}$ by setting

$$
(\alpha 1+x, \beta 1+y)=(x, y)-\alpha \beta,
$$

$x, y$ in $\Im_{0}$. Let $a \in \mathfrak{\Im}_{0}$ and let $R_{a}$ denote the multiplication $x \rightarrow x a$ in $\mathfrak{\Im}$ determined by $a$. Then $1 a=a$ and $x a=(x, a) 1$ if $x \in \mathfrak{I}_{0}$; hence $R_{a}$ coincides with the mapping $x \rightarrow(x, a) 1-(x, 1) a$. Moreover, $\left[R_{a} R_{b}\right]$, $a, b \in \Im_{0}$, is the linear mapping $x \rightarrow(x, a) b-(x, b) a$. It follows from 
this that the set $\mathscr{L}=\left\{R_{a}+\sum\left[R_{b_{i}} R_{c_{i}}\right] \mid a, b_{i}, c_{i} \in \Im_{0}\right\}$ is the Lie algebra of linear transformations (in $\Im$ ) which are skew relative to the symmetric scalar product $(x, y)$ in $\Im$ in the sense that $(x T, y)=-(x, y T)$ for all $x, y$. If $a, b, c \in \mathfrak{\Im}_{0}$ then, by (9),

$$
\left[\left[R_{a} R_{b}\right] R_{c}\right]=R_{a}, \quad d=(b, c) a-(a, c) b .
$$

We can now prove the following useful result.

Proposition 13. 3. Let $\Im$ be the Jordan algebra of a non-degenerate symmetric scalar product and let $\mathfrak{Q}$ denote the Lie algebra of linear transformations in $\mathfrak{F}$ which is generated by the multiplications $R_{a}$, $a \in \mathfrak{\Im}_{0}$. Then $\mathbb{Q}$ is simple if $n \geqslant 5$. Next suppose that for each $a \in \mathfrak{S}_{0}$ we have defined a linear transformation $U_{a}$ in some vector space and that $U_{a}$ is linear in $a$ and satisfies

$$
\left[\left[U_{a} U_{b}\right] U_{c}\right]=U_{a}, \quad d=(b, c) a-(a, c) b .
$$

Then $R_{a} \rightarrow U_{a}$ defines a homomorphism of $\&$ onto the Lie algebra generated by the $U_{a}$.

Proof. Since $\mathbb{Z}$ is the set of skew transformations relative to a non-degenerate scalar product, the statement on the simplicity follows from known results. ${ }^{17)}$ It is also well known that the dimensionality of this Lie algebra is $n(n+1) / 2=n+n(n-1) / 2$. Hence if the elements $u_{i}, i=1, \cdots, n$, form a basis for $\Im_{0}$, then the elements $R_{u_{i}},\left[R_{u_{i}} R_{u_{j}}\right]$ where $i<j$ form a basis for $\mathbb{R}$. One verifies directly that the linear mapping which sends $R_{u_{i}}$ into $U_{u_{i}}$ and $\left[R_{u_{i}} R_{u_{j}}\right]$ into $\left[U_{u_{i}} U_{u_{j}}\right]$ is a homomorphism of $\mathfrak{Q}$ onto the Lie algebra generated by the $U_{a}$.

\section{Structure of the Clifford algebra}

Let $\Im$ be the Jordan algebra of the symmetric scalar product $(x, y)$. Then the universal associative algebra of special representations $\mathfrak{u}_{s}(\mathfrak{S})$ is called the Clifford algebra of $(x, y)$. In particular, if $(x, y) \equiv 0$ then $\mathfrak{u}_{s}(\mathfrak{\Im})$ is the Grassmann (or exterior) algebra of the vector space $\Im_{0}$. We proceed to determine the structure of $\mathfrak{u}_{s}(\mathfrak{\Im})$.

Theorem 14.1. Let $\Im$ be the Jordan algebra of $(x, y)$ and assume that $\operatorname{dim} \mathfrak{\Im}=n+1$. Then (1) the dimensionality of the Clifford algebra $\mathfrak{u}_{s}(\mathfrak{\Im})$ is $2^{n},(2)$ the ideal in $\mathfrak{u}_{s}(\Im)$ generated by the radical of $(x, y)$ is the radical of $\mathfrak{U}_{s}(\mathfrak{\Im}),(3) \mathfrak{U}_{s}(\mathfrak{\Im})$ is a direct sum of its radical and $a$

17) For characteristic 0 this is well known. For characteristic $p(\neq 0)$ it is proved in [17], p. 497 . 
subalgebra which is isomorphic to the Clifford algebra of the nondegenerate symmetric scalar product determined by $(x, y)$. (4) If $(x, y)$ is non-degenerate and $n$ is even, then $\mathfrak{H}_{s}(\mathfrak{\Im})$ is central simple. If $n$ is odd $\mathfrak{u}_{s}(\mathfrak{\Im})$ is either simple with center a quadratic extension of the base field or is a direct sum of two central simple algebras. These two possibilities occur according as $(-1)^{\left[\frac{n}{2}\right]} \delta$ is not or is a square in $\Phi$ for $\delta$ the discriminant of $(x, y){ }^{18)}$

Proof. We can choose a basis $\left(u_{1}, u_{2}, \cdots, u_{n}\right)$ for $\Im_{0}$ so that

$$
\left(u_{i}, u_{j}\right)=\delta_{i j} \beta_{i} ; \quad \beta_{i} \neq 0, \quad i \leqslant r ; \quad \beta_{j}=0, j>r .
$$

Then the space spanned by $u_{r+1}, \cdots, u_{n}$ is the radical of $(x, y)$. We now denote by $\bar{a}$ (rather than $a_{s}$ ) the coset in $\mathfrak{u}_{s}(\Im)$ determined by the element $a \in \Im$. Then the elements $\bar{u}_{1}, \bar{u}_{2}, \cdots, \bar{u}_{n}$ and $\bar{u}_{0} \equiv 2 \cdot \overline{1}$ generate the Clifford algebra. $\bar{u}_{0}$ is the identity of this algebra and we have the relations

$$
\bar{u}_{i} \bar{u}_{j}+\bar{u}_{j} \bar{u}_{i}=\frac{1}{2} \delta_{i j} \beta_{i} \bar{u}_{0}
$$

or

$$
\bar{u}_{i} \bar{u}_{j}=-\bar{u}_{j} \bar{u}_{i}, \quad i \neq j ; \quad u_{i}^{2}=\gamma_{i} u_{0}, \quad \gamma_{i}=\frac{1}{4} \beta_{i} .
$$

It follows that every element of the Clifford algebra is a linear combination of the $2^{n}$ elements $\bar{u}_{0}, \bar{u}_{i_{1}} \bar{u}_{i_{2}} \cdots \bar{u}_{i_{k}}, i_{1}<i_{2}<\cdots<i_{k}$, $i_{j}=1,2, \cdots, n$. It is not difficult to prove that these elements are linearly independent. (This is more or less well known.) Hence (1) holds. The ideal $\mathfrak{R}$ generated by $\Re$ has the basis $\left\{\bar{u}_{i_{1}} \bar{u}_{i_{2}} \cdots \bar{u}_{i_{k}}\right.$ $\left.\mid i_{1}<i_{2}<\cdots<i_{k}, i_{k}>r\right\}$. Since the squares of these elements are 0 and any two either commute or anti-commute, $\mathfrak{N}$ is a nil ideal. Evidently, $\mathfrak{U}_{s}(\mathfrak{I})=\mathfrak{R} \oplus \mathfrak{U}_{s}(\mathfrak{B})$ where $\mathfrak{B}$ is the Jordan algebra determined by $(x, y)$ and the space $\mathfrak{B}_{0}$ spanned by $\left(\bar{u}_{1}, \bar{u}_{2}, \cdots, \bar{u}_{r}\right)$. Since $(x, y)$ is non-degenerate in $\mathfrak{B}_{0},(2)$ and $(3)$ are consequences of (4). We now assume that $(x, y)$ is non-degenerate. Then $r=n$ and all the $\gamma_{i} \neq 0$. In this case it is well known that if $n$ is even then $\mathfrak{H}_{s}(\mathfrak{\Im})$ is a Kronecker product of (generalized) quaternion algebras while if $n$ is odd then $\mathfrak{u}_{s}(\mathfrak{F})$ has a center with basis $1, c$ where $c=\bar{u}_{1} \bar{u}_{2} \cdots \bar{u}_{n}$ and $c^{2}=(-1)^{\left[\frac{n}{2}\right]}{ }_{\gamma_{1} \gamma_{2} \cdots \gamma_{n} \cdot{ }^{19)}}$ Also in the this case $\mathfrak{u}_{s}(\mathfrak{\xi})$ is a Kronecker product of $\Phi(c)$ and quaternion algebras. Now $\Phi(c)$ is either a quadratic field or a direct sum of two copies of $\Phi$ according as

18) This result is well known. We include the proof for the sake of completeness.

19) Cf., for example, [15], p. 155. 
$(-1)^{\left[\frac{n}{2}\right]} \gamma_{1} \cdots \gamma_{n}$ is not or is a square. Since the discriminant of $(x, y)$ is $\beta_{1} \beta_{2} \cdots \beta_{n}$ and $\beta_{i}=4 \gamma_{i},(4)$ is now clear.

Since the elements $\bar{u}_{i}$ are linearly independent it is clear that the mapping $a \rightarrow \bar{a}$ of $\mathfrak{\Im}$ into $\mathfrak{U}_{s}(\mathfrak{\Im})$ is $1-1$. This remark shows that $\Im$ is a special Jordan algebra. In the sequel we shall not distinguish between the elements of $\mathfrak{s}$ and their images in $\mathfrak{u}_{s}(\mathfrak{\Im})$. We shall therefore drop the bars in the notation introduced in the above proof.

The structure of the Clifford algebra $\mathfrak{u}_{\boldsymbol{s}}(\mathfrak{\Im})$ and the known theory of representations of associative algebras give a complete solution of the problem of special representations of the Jordan algebra $\Im$ of a non-degenerate symmetric scalar product. Thus the semi-simplicity of the $\mathfrak{U}_{s}(\Im)$ implies complete reducibility of the special representations. If $n$ is even we have just one irreducible representation (in the sense of isomorphism). while if $n$ is odd we have either one or two according as $(-1)^{\left[\frac{n}{2}\right]}$ is not or is a square in $\Phi$.

\section{Meson algebras}

We have noted $(\S 5)$ that the universal associative algebra $\mathfrak{u}(\Im)$ of the representations of a Jordan algebra with an identity is a direct sum of the universal algebra $\mathfrak{u}_{s}(\mathfrak{\Im})$ of the special representations of $\mathfrak{\Im}$ and the universal algebra $\mathfrak{u}_{1}(\mathfrak{\Im})$ of the unital representations. Also we have a homomorphism of $\mathfrak{u}_{1}(\mathfrak{\Im})$ onto $\mathfrak{u}_{s}^{(2)}(\mathfrak{\Im})$ the universal associative algebra of sums of commuting special representations. We proceed now to the analysis of $\mathfrak{U}_{1}(\mathfrak{\Im})$ for the Jordan algebra $\Im$ of a symmetric scalar product. Since these algebras seem to have

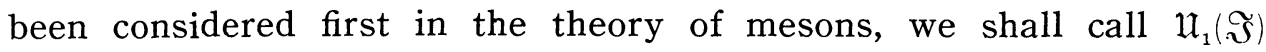
the meson algebra of the symmetric scalar product $(x, y)$ (in $\mathfrak{\Im}_{0}$ ). If we identify $\mathfrak{\Im}_{0}$ with its corresponding subset in $\mathfrak{U}_{1}(\mathfrak{\Im})$ then we see that $\mathfrak{u}_{1}(\Im)$ is generated by 1 and the elements of $\Im_{0}$ and that the latter satisfy the relations

$$
x y z+z y x=(x, y) z+(y, z) x .
$$

We determine first an upper bound for the dimensionality of $\mathfrak{u}_{1}(\mathfrak{\Im})$.

LEMmA. If $\operatorname{dim} \Im=n+1$ then $\operatorname{dim} \mathfrak{u}_{1}(\mathfrak{\Im}) \leqslant\left(\begin{array}{c}2 n+1 \\ n\end{array}\right)$.

Proof. Let $\left(u_{1}, u_{2}, \cdots, u_{n}\right)$ be a basis for $\mathfrak{\Im}_{0}$. Then it follows easily from $(80)$ that every element of $\mathfrak{u}_{1}(\mathfrak{\Im})$ is a linear combination of 1 and the elements

$$
u_{i_{1}}^{2} u_{i_{2}}^{2} \cdots u_{i_{k}}^{2} u_{i_{k+1}} u_{i_{k+2}} \cdots u_{i_{l}}
$$


where

$$
i_{1}<i_{2}<\cdots<i_{k} ; \quad i_{k+1}<i_{k+3}<\cdots ; \quad i_{k+2}<i_{k+4}<\cdots .
$$

The number of such elements is

$$
N=\sum_{l=0}^{n}\left(\begin{array}{l}
n \\
l
\end{array}\right)\left(\sum_{k=0}^{l}\left(\begin{array}{l}
l \\
k
\end{array}\right)\left(\begin{array}{l}
l-k \\
{\left[\frac{1}{2}(l-k)\right]}
\end{array}\right) .\right.
$$

Since $\left(\begin{array}{l}l \\ k\end{array}\right)\left(\begin{array}{l}l-k \\ {\left[\frac{1}{2}(l-k)\right]}\end{array}\right)=\frac{l !}{k !\left(\frac{l-k}{2}\right) !\left(\frac{l-k}{2}\right) !} \quad$ if $\quad l-k \quad$ is $\quad$ even $\quad$ and $=\frac{l !}{k !\left(\left[\frac{l-k}{2}\right]+1\right) !\left[\frac{l-k}{2}\right] !}$ if $l-k$ is odd, the sum $f(l) \equiv \sum_{k=0}^{l}\left(\begin{array}{l}l \\ k\end{array}\right)\left(\begin{array}{l}l-k \\ {\left[\frac{1}{2}(l-k)\right]}\end{array}\right)$

is the sum of the coefficients of the terms in $(a+b+c)^{b}$ for which the exponents of $b$ and $c$ are equal and those for which the exponent of $b$ exceeds that of $c$ by 1 . This is the same as the sum of the constant term and the coefficient of $b$ in $\left(1+b+\frac{1}{b}\right)^{l}$. Hence $N$ is the sum of the constant term and the coefficient of $b$ in

$$
\left(2+b+\frac{1}{b}\right)^{n}=\sum_{l=0}^{n}\left(\begin{array}{l}
n \\
l
\end{array}\right)\left(1+b+\frac{1}{b}\right)^{l} .
$$

This is the same as the sum of the coefficients of $b^{n}$ and $b^{n+1}$ in $\left(b^{2}+2 b+1\right)^{n}=(b+1)^{2 n}$. Thus

$$
N=\left(\begin{array}{c}
2 n \\
n
\end{array}\right)+\left(\begin{array}{c}
2 n \\
n+1
\end{array}\right)=\left(\begin{array}{c}
2 n+1 \\
n
\end{array}\right)
$$

and $\operatorname{dim} \mathfrak{u}_{1}(\mathfrak{\Im}) \leq\left(\begin{array}{c}2 n+1 \\ n\end{array}\right)$.

Theorem 15.1. If $\mathfrak{\Im}$ is the Jordan algebra of the symuetric scalar product $(x, y)$ and $\operatorname{dim} \mathfrak{\Im}=n+1$, then the dimensionality of the meson algebra $\mathfrak{H}_{1}(\mathfrak{\Im})$ is $\left(\begin{array}{c}2 n+1 \\ n\end{array}\right)$ and $\mathfrak{U}_{1}(\mathfrak{\Im})$ is isomorphic to the universal associative algebra $\mathfrak{H}_{s}^{(2)}(\Im)$ of sums of commuting special representation of $\Im$.

Proof. Since we have a homomorphism of $\mathfrak{U}_{1}(\mathfrak{\Im})$ onto $\mathfrak{u}_{s}^{(2)}(\mathfrak{\Im})$ and since $\operatorname{dim} \mathfrak{u}_{1} \leqslant\left(\begin{array}{c}2 n+1 \\ n\end{array}\right)$, it suffices to show that $\operatorname{dim} \mathfrak{u}_{s}^{(2)}=\left(\begin{array}{c}2 n+1 \\ n\end{array}\right)$. We choose a basis for $\mathfrak{\Im}_{0}$ so that $\left(u_{i}, u_{j}\right)=\delta_{i j} \beta_{j}$. Then (79) holds for the corresponding elements of $\mathfrak{u}_{s}(\mathfrak{S})$ and the elements $u_{i_{1}} u_{i_{2}} \cdots u_{i_{k}}$, $1 \leqslant i_{1}<i_{2}<\cdots$ form a basis for $\mathfrak{u}_{s}(\mathfrak{\Im})$. (We now adopt the convention that this product represents 1 if $k=0$.) The elements $u_{i_{1}} u_{i_{2}} \cdots u_{i_{k}}$ $\times u_{j_{1}} u_{j_{2}} \cdots u_{j_{l}}, i_{1}<i_{2}<\cdots, j_{1}<j_{2}<\cdots$ form a basis for $\mathfrak{u}_{s} \times \mathfrak{u}_{s}$, and 
the elements $v_{i}=u_{i} \otimes u_{0}+u_{0} \otimes u_{i}, i=1,2, \cdots, n$, and 1 ( $\left.=u_{0} \times u_{0}\right)$ are generators for $\mathfrak{U}_{s}^{(2)}$. Every element of $\mathfrak{U}_{s}^{(2)}$ is a linear combination of the $\left(\begin{array}{c}2 n+1 \\ n\end{array}\right)$ elements $v_{i_{1}}^{2} v_{i_{2}}^{2} \cdots v_{i_{k}}^{2} v_{i_{k+1}} \cdots v_{i_{l}}$ where the $i_{j}$ are distinct and satisfy (82). It remains to see that these elements are linearly independent. Hence suppose we have a non-trivial linear relation connecting these elements. Let $v_{r}$ be one of the $v$ 's and write the relation as $A_{2}+A_{1}+A_{0}=0$ where $A_{i}$ is homogeneous of degree $i$ in $v_{r}$. Since $v_{r}^{2} v_{i}^{2}=v_{i}^{2} v_{r}^{2}$ for all $i$ we can write $A_{2}=v_{r}^{2} B_{0}$ where $B_{0}$ is homogeneous of degree 0 in $v_{r}$. Now any element which is homogeneous of degree 0 in $v_{r}$ can be expressed in terms of the base elements $u_{i_{1}} \cdots u_{i_{s}} \otimes u_{j_{1}} \cdots u_{j_{l}}$ with indices $\neq r$. If the coefficient of $u_{i_{1}} \cdots u_{i_{k}}$ $\otimes u_{j_{1}} \cdots u_{j_{l}}$ is $\neq 0$ the same holds for the coefficient of $u_{i_{1}} \cdots u_{r} \cdots u_{i_{k}}$ $\otimes u_{j_{1}} \cdots u_{r} \cdots u_{j_{l}}$ in $v_{r}^{2}$ times the given homogeneous element. It follows that $B_{0}=0$. A similar argument shows that $A_{0}=0$; hence also $A_{1}=0$. This reasoning shows that the existence of a non-trivial linear relation connecting the $v_{i_{1}}^{2} \cdots v_{i_{k}}^{2} v_{i_{k+1}} \cdots v_{i_{l}}$ implies the existence of such a relation which is homogeneous of degree 1 in, say, $v_{1}, v_{2}, \cdots, v_{t}$ and of degree 0 in the remaining $v$ 's. Such a relation has the form $\sum \alpha_{j_{1}} \cdots j_{t} v_{j_{1}} v_{j_{2}} \cdots v_{j_{t}}=0$ where the summation is taken over all the permutations $j_{1}, j_{2}, \cdots, j_{t}$ of $1,2, \cdots, t$ such that $j_{1}<j_{3}<\cdots$ and $j_{2}<j_{4}<\cdots$. We assume also that our non-trivial relation is shortest and that $t$ is minimal. Now $v_{1}$ must occur either in the first or the second place in the terms of the relation. Since $v_{1} v_{i} v_{1}=0$ for $i \neq 1$, left multiplication of the relation by $v_{1}$ annihilates the terms in which $v_{1}$ occurs in second position. The remaining terms give a relation $\Sigma^{\prime} \alpha_{j_{1}} \cdots j_{t} v_{1}^{2} v_{j_{2}} \cdots v_{j_{t}}=0$ and $\Sigma^{\prime} \alpha_{j_{1}} \cdots j_{b} v_{j_{2}} \cdots v_{j_{t}}$ $=0$. This contradicts the assumption made on the degree of the original relation unless the new relation is trivial. Thus we see that in the first relation $v_{1}$ occurs in the second position for every term with non-zero coefficient. For these terms $v_{2}$ occurs in either the first or fourth positions. Left multiplication by $v_{2}$ shows that $v_{2}$ occurs in the fourth position in every term with non-zero coefficient. Continuing in this way we see that the terms with non-zero coefficient have the form $* v_{1} * v_{2}^{*} v_{3} * \ldots$. Evidently there is only one such term, and if we express the $v_{i}$ as $u_{i} \otimes v_{0}+u_{0} \otimes u_{i}$ we see that it is $\neq 0$. Thus we have a contradiction and the theorem is proved.

\section{Structure of meson algebras}

It is easy to see that the radical of $(x, y)$ generates a nil-ideal in the meson algebra $\mathfrak{U}_{1}(\mathfrak{\Im})=\mathfrak{U}_{s}^{(2)}(\mathfrak{\Im})$ and that $\mathfrak{u}_{1}$ is a direct sum of this 
ideal and a subalgebra which is the algebra associated with the nondegenerate scalar product determined by $(\boldsymbol{x}, \boldsymbol{y})$. We therefore obtain, as usual, a reduction to the non-degenerate case. From now on we assume that $(x, y)$ is non-degenerate. Suppose first that $n\left(=\operatorname{dim} \mathfrak{\Im}_{0}\right)$ is even.

Theorem 16.1. Let $(x, y)$ be a non-degenerate symmetric scalar product in a space of even dimensionality $n$ and let $\mathfrak{H}_{1}$ be the meson algebra associated with $(x, y)$. Then $\mathfrak{u}_{1}$ is a direct sum of ideals which are matrix algebras $\Phi_{k}, k=\left(\begin{array}{c}n+1 \\ 1\end{array}\right),\left(\begin{array}{c}n+1 \\ 3\end{array}\right), \cdots,\left(\begin{array}{l}n+1 \\ n+1\end{array}\right)$.

Proof. Consider the Clifford algebra $\mathfrak{U}_{s}$. We have seen (Th. 14.1) that this is central simple. If $x \in \mathfrak{U}_{s}$ we denote the left (right) multiplication in the $\mathfrak{u}_{s}$ determined by $x$ as $L_{x}\left(R_{x}\right)$. Since $\mathfrak{u}_{s}$ is central simple the algebra generated by the $L_{x}$ and $R_{y}$ is the Kronecker product of the algebra generated by the $L_{x}$ and that generated by the $R_{y} .{ }^{21)} \quad$ If $a \in \mathfrak{\Im}\left(\subseteq \mathfrak{U}_{s}\right)$ then $a \rightarrow L_{a}$ and $a \rightarrow R_{a}$ are commuting special representations. Hence $a \rightarrow L_{a}+R_{a}$ is a unital representation of $\Im$. The enveloping associative algebra of this representation has the same dimensionality $\left(\begin{array}{c}2 n+1 \\ n\end{array}\right)$ as $\mathfrak{u}_{1}$ (cf. the proof of Th. 15.1). Hence it gives a faithful representation of the meson algebra. We shall now define certain invariant subspaces of the Clifford algebra relative to the transformations $L_{a}+R_{a}, a \in \Im$. For this purpose we choose a basis $u_{i}, i=1,2, \cdots, n$, for $\Im_{0}$ such that $\left(u_{i}, u_{j}\right)=\delta_{i j} \beta_{i}$. Let $\Im^{(k)}, k=0,1, \cdots, n$, be the space spanned by the vectors $u_{i_{1}} u_{i_{2}} \cdots u_{i_{k}}, i_{1}<i_{2}<\cdots<i_{k}$. Then one verifies that the subspaces $\Im^{(0)}+\Im^{(1)}, \Im^{(2)}+\Im^{(3)}, \cdots, \Im^{(n)}$ are invariant relative to the $L_{a}+R_{a}$. Typical relations for this are the following:

$$
\begin{aligned}
& \left\{u_{1} \cdots u_{2 h-2}, u_{2 h-1}\right\}=2 u_{1} u_{2} \cdots u_{2 h-1} \\
& \left\{u_{i} \cdots u_{2 h-2}, u_{2 h-2}\right\}=0 \\
& \quad\left\{u_{1} \cdots u_{2 h-1}, u_{2 h}\right\}=0 \\
& \left\{u_{1} \cdots u_{2 h-1}, u_{2 h-1}\right\}=\rho u_{1} \cdots u_{2 h-2}, \quad \rho \neq 0 .
\end{aligned}
$$

Since the dimensionality of $\Im^{(k)}$ is $\left(\begin{array}{l}n \\ k\end{array}\right)$, the dimensionalities of $\mathfrak{\Im}^{(0)}+\mathfrak{F}^{(1)}, \Im^{(2)}+\mathfrak{\Im}^{(3)}, \cdots$ are respectively $\left(\begin{array}{c}n+1 \\ 1\end{array}\right),\left(\begin{array}{c}n+1 \\ 3\end{array}\right), \ldots$. Hence

20) For the case of an algebraically closed base field, this theorem as well as Theorem 16.2 are due to Svartholm [34]. Cf. also Jacobson [20].

21) See, for example, the author's Theory of Rings, New York, 1943, p. 103. 
the dimensionality of the enveloping associative algebra of the $L_{a}+R_{a}$ is at most

$$
\left(\begin{array}{c}
n+1 \\
1
\end{array}\right)^{2}+\left(\begin{array}{c}
n+1 \\
3
\end{array}\right)^{2}+\cdots+\left(\begin{array}{c}
n+1 \\
n+1
\end{array}\right)^{2}=\left(\begin{array}{c}
2 n+1 \\
n
\end{array}\right)
$$

Now this bound can be attained only if the enveloping algebra of the induced linear transformations in $\mathfrak{\Im}^{(0)}+\mathfrak{F}^{(1)}, \mathfrak{\Im}^{(2)}+\mathfrak{\Im}^{(3)}, \cdots$ are the complete algebras of linear transformations and the enveloping algebra of the $L_{a}+R_{a}$ is the direct sum of these algebras. Since we have observed that the enveloping associative algebra of the $L_{a}+R_{a}$ does have dimension $\left(\begin{array}{c}2 n+1 \\ n\end{array}\right)$, this proves that it is the direct sum indicated. Hence the meson algebra has this structure.

The discussion of the case in which $n=2 \nu-1$ is odd is more complicated owing to the fact that the Clifford algebra is not central simple in this case. In order to circumvent this difficulty we imbed the odd dimensional space $\mathfrak{\Im}_{0}$ in an even dimensional one $\Re_{0}=\Im_{0}+\Phi u_{n+1}$ with a scalar product which is an extension of the given one. We may assume also that $\left(u_{n+1}, u_{n+1}\right)=1$ and $\left(u_{n+1}, \mathfrak{\Im}_{0}\right)=0$. Let $\Omega$ be the Jordan algebra $\Re_{0} \oplus \Phi 1$ and let $\mathfrak{H}_{s}(\Omega)$ be the associated Clifford algebra. Then $\Re \supseteq \mathfrak{\Im}$ and $\mathfrak{u}_{s}(\Re) \supseteq \mathfrak{U}_{s}(\mathfrak{\Im})$. Let $L_{a}, R_{a}, a \in \mathfrak{\Im}$, now denote the left and right multiplication, respectively, acting in $\mathfrak{u}_{s}(\Re)$. Then $a \rightarrow L_{a}+R_{a}$ is a unital representation of $\Im$ whose enveloping associative algebra is isomorphic to the meson algebra $\mathfrak{U}_{1}(\mathfrak{\Im})$ of $\Im$. As before, we wish to decompose $\mathfrak{u}_{s}(\Re)$ into subspaces which are invariant relative to the $L_{a}+R_{a}$. Let $\mathfrak{\Im}^{(k)}, k=0, \cdots, n$, be the subspace spanned by the vectors $u_{i_{1}} u_{i_{2}} \cdots u_{i_{k}}$ where the $i_{j}$, are in increasing order and have values in $1, \cdots, n$. Then the subspaces $\Im^{(1)}+\Im^{(1)}$, $\Im^{(2)}+\Im^{(3)}, \cdots, \Im^{(n-1)}+\Im^{(n)}$ and $\Im^{(0)} u_{n+1}, \Im^{(1)} u_{n+1}+\Im^{(2)} u_{n+1}, \Im^{(3)} u_{n+1}$ $+\Im^{(4)} \dot{u}_{n+1}, \cdots, \Im^{(n)} u_{n+1}$ are invariant relative to $L_{a}+R_{a}, a \in \Im$. This can be verified by straight-forward calculations like those in (85). Since $c=u_{1} u_{2} \cdots u_{n}$ commutes with the $u_{i}$, the linear transformation $C=L_{c}$ commutes with the $L_{a}+R_{a}$. Since this mapping is $1-1$ and sends $\mathfrak{F}^{(0)}+\mathfrak{F}^{(1)}$ onto $\mathfrak{\Im}^{(n-1)}+\mathfrak{J}^{(n)}, \mathfrak{F}^{(2)}+\mathfrak{\Im}^{(3)}$ onto $\mathfrak{\Im}^{(n-3)}+\mathfrak{F}^{(n-2)} \ldots$ and $\Im^{(0)} u_{n+1}$ onto $\Im^{(n)} u_{n+1}, \Im^{(1)} u_{n+1}+\mathfrak{\Im}^{(2)} u_{n+1}$ onto $\mathfrak{\Im}^{(n-2)} u_{n+1}+\mathfrak{\Im}^{(n-1)} u_{n+1}, \cdots$, it defines an isomorphism between these spaces. Hence in seeking representatives of the classes of isomorphic spaces we are led to consider only the following spaces:

$$
\begin{aligned}
& \Im^{(0)}+\Im^{(1)}, \Im^{(2)}+\Im^{(3)}, \cdots, \Im^{(\nu-2)}+\Im^{(\nu-1)} \\
& \Im^{(0)} u_{n+1}, \Im^{(1)} u_{n+1}+\Im^{(2)} u_{n+1} \cdots \Im^{(\nu-1)} u_{n+1}+\Im^{(\nu)} u_{n+1}
\end{aligned}
$$

if $\nu$ is even and 


$$
\begin{aligned}
& \Im^{(0)}+\Im^{(1)}, \Im^{(2)}+\Im^{(3)}, \cdots, \Im^{(\nu-1)}+\Im^{(\nu)} \\
& \Im^{(0)} u_{n+1}, \Im^{(1)} u_{n+1}+\Im^{(2)} u_{n+1}, \cdots, \Im^{(\nu-2)} u_{n+1}+\Im^{(\nu-1)} u_{n+1}
\end{aligned}
$$

if $\nu$ is odd. In the first case $C$ maps $\Im^{(\nu-1)} u_{n+1}+\mathfrak{F}^{(\nu)} u_{n+1}$ into itself and in the second it maps $\mathfrak{\Im}^{(\nu-1)}+\mathfrak{F}^{(\nu)}$ into itself. In either case $C$ interchanges the two spaces displayed and these spaces have the same dimensionality $\left(\begin{array}{c}n \\ \nu-1\end{array}\right)=\left(\begin{array}{c}n \\ \nu\end{array}\right)=\frac{1}{2}\left(\begin{array}{c}n+1 \\ \nu\end{array}\right)$. Suppose now that $(-1)^{\left[\frac{n}{2}\right]} \delta$, $\delta$ the discriminant of $(x, y)$ is a square. In this case we can replace $C$ by a suitable scalar times $C$ and obtain $D$ such that $D^{2}=1$. It follows that the space $\Im^{(\nu-1)} u_{n+1}+\Im^{(\nu)} u_{n+1}, \nu$ even, and $\Im^{(\nu-1)}+\Im^{(\nu)}, \nu$ odd, decomposes as a direct sum of two invariant subspaces relative to the $L_{a}+R_{a}$ and that these spaces have the same dimensionality $\frac{1}{2}\left(\begin{array}{c}n+1 \\ \nu\end{array}\right)$. Thus the maximum dimensionality of the induced set of linear transformations in $\Im^{(\nu-1)} u_{n+1}+\Im^{(\nu)} u_{n+1}$ or $\Im^{(\nu-1)}+\mathfrak{F}^{(\nu)}$ by the enveloping associative algebra of the $L_{a}+R_{a}$ is $\frac{1}{2}\left(\begin{array}{c}n+1 \\ \nu\end{array}\right)^{2}$. For the other subspaces in $(83)$ and $\left(83^{\prime}\right)$ we obtain the upper bounds $\left(\begin{array}{c}n+1 \\ 0\end{array}\right)^{2}$, $\left(\begin{array}{c}n+1 \\ 1\end{array}\right)^{2}, \cdots,\left(\begin{array}{c}n+1 \\ \nu-1\end{array}\right)^{2}$. Now $\sum_{i=0}^{\nu-1}\left(\begin{array}{c}n+1 \\ i\end{array}\right)^{2}+\frac{1}{2}\left(\begin{array}{c}n+1 \\ \nu\end{array}\right)^{2}=\left(\begin{array}{c}2 n+1 \\ n\end{array}\right)$. Hence it follows that the induced sets of linear transformations in the spaces indicated are the complete sets of linear transformations in these spaces. This implies that the meson algebra is a direct sum of ideals which are matrix algebras $\Phi_{k}, k=\left(\begin{array}{c}n+1 \\ 0\end{array}\right), \cdots,\left(\begin{array}{c}n+1 \\ \nu-1\end{array}\right)$ and two matrix algebras $\Phi_{k^{\prime}}, k^{\prime}=\frac{1}{2}\left(\begin{array}{c}n+1 \\ \nu\end{array}\right)$. Suppose next that $(-1)^{\left[\frac{n}{2}\right]} \delta$ is not a square. Then our reasoning shows that if we adjoin the square root of this element then the induced algebra of linear transformations in $\Im^{(\nu-1)} u_{n+1}+\Im^{(\nu)} u_{n+1}$, $\nu$ even, or in $\Im^{(\nu-1)}+\Im^{(\nu)}$, $\nu$ odd, is a direct sum of two matrix algebras of ${k^{\prime}}^{2}$ dimensions. Hence the original induced set of linear transformations is an algebra which is either a direct sum of two copies of $\Phi_{k^{\prime}}$ or is of the form $\mathrm{P}_{k^{\prime}}$ where $\mathrm{P}$ is a quadratic field over $\Phi$. Since there exists a linear transformation $C$ which is not a scalar but whose square is a scalar commuting with the algebra of induced linear transformations in $\Im^{(\nu-1)} u_{n+1}+\mathfrak{F}^{(\nu)} u_{n+1}$ or $\Im^{(\nu-1)}+\Im^{(\nu)}$ it follows that only the latter alternative can hold and that $\mathrm{P}=\Phi(c)$. If we observe that in the first case $\left((-1)^{\left[\begin{array}{l}n \\ 2\end{array}\right]} \delta\right.$ is a square $\Phi[c]$ is a direct sum of two algebras of order one, then we can state the result in a uniform way as follows

Theorem 16.2. Let $(x, y)$ be a non-degenerate symmetric scalar product in a space of odd dimensionality $n=2 \nu-1$. Then the meson algebra 
$\mathfrak{u}_{1}$ of $(x, y)$ is a direct sum of ideals which are matrix algebras $\Phi_{k}, k=\left(\begin{array}{c}n+1 \\ 0\end{array}\right),\left(\begin{array}{c}n+1 \\ 1\end{array}\right), \cdots,\left(\begin{array}{c}n+1 \\ \nu-1\end{array}\right), \quad$ and $\quad \mathrm{P}_{k^{\prime}} \quad k^{\prime}=\frac{1}{2}\left(\begin{array}{c}n+1 \\ \nu\end{array}\right) \quad \mathrm{P}$ the center of the Clifford algebra of $(x, y)$.

We remark that the numbers $k$ occuring in Th. 16.1 can also be written as $\left(\begin{array}{c}n+1 \\ 0\end{array}\right),\left(\begin{array}{c}n+1 \\ 1\end{array}\right), \cdots,\left(\begin{array}{c}n+1 \\ \nu\end{array}\right)$ so that we can state that result in a manner similar to that of Th. 16.2. It is note-worthy that the structure of the meson algebra does not depend very much on the nature of the scalar product. In the even dimensional case, it is a function of the dimensionality only while in the odd dimensional case it depends only on the dimensionality and the discriminant. This is not too surprising since it is related to the well-known fact that the structure of the algebra generated by the left and right multiplications in a simple associative algebra depends only on the dimensionality and the center.

Since the meson algebra is semi-simple, it follows that every unital representation of the Jordan algebra $\mathfrak{\Im}$ is completely reducible. The number and nature of the irreducible unital representations are given in Ths. 16.1 and 16.2. Thus these are just the representations induced in the various subspaces which we used in proving these theorems. It is clear also that these representations are induced representations in the sum of the two commuting special representations $a \rightarrow L_{a}, a \rightarrow R_{a}$ (in $\mathfrak{U}_{s}(\mathfrak{I})$ or $\mathfrak{U}_{s}(\mathfrak{R})$ ). This and the complete reducibility imply the following result.

Theorem 16.3. Every unital module of the Jordan algebra i of a non-degenerate symmetric scalar product is a submodule of a sum of two commuting special modules.

IV. Representation of Finite Dimensional Semi-Simple Alternative AND Jordan Algebras

17. Resume and remarks on the structure theory

The structure theory of finite dimensional alternative algebras is due to Zorn ([37]), that of finite dimensional Jordan algebras to Albert([2] and [4]). In both cases the principal facts are as follows. If $\mathfrak{A}$ is finite dimensional alternative or Jordan, $\mathfrak{A}$ contains a unique maximal nilpotent ideal $\mathfrak{N}$ called the radical of $\mathfrak{A}$. The difference algebra $\mathfrak{A} / \mathfrak{R}$ is semi-simple in the sense that its radical is 0 . Every semi-simple algebra is a direct sum of ideals which are simple algebras (with non-zero squares). It remains to list the simple algebras 
and it suffices to consider those which are central.

If $\mathfrak{A}$ is an alternative simple algebra, either $\mathfrak{A}$ is associative, in which case, its structure is known by the Wedderburn theorem, or $\mathfrak{A}$ is a (generalized) Cayley algebra C. Such an algebra is obtained by starting with a quaternion algebra $Q$ and forming the direct sum of $Q$ and a Cayley bimodule for $Q$ obtained from the right regular representation. (Cf p. 42 of these proofs. One takes $a \rightarrow U_{a}$ to be the right regular representation.) Multiplication of elements in $Q$ by those of the module is given by the module compositions. If $u$ is an element of the module corresponding to 1 then the module is $Q u=\{q u \mid q \in Q\}$. Hence we have the rules:

$$
q_{1}\left(q_{2} u\right)=\left(q_{2} q_{1}\right) u, \quad q u=u \vec{q},\left(u q_{1}\right) q_{2}=u\left(q_{2} q_{1}\right)
$$

where the $q_{i} \in Q$ and $\vec{q}=\operatorname{tr} q-q$ the usual conjugate quaternion. To complete the multiplication one must define $\left(q_{1} u\right)\left(q_{2} u\right)$. The alternative identities give $q u^{2}=u^{2} q$ and this implies that $u^{2}=\mu 1$. One can then deduce from the Moufang identity $\left(u \bar{q}_{1}\right)\left(q_{2} u\right)=\left(u\left(\vec{q}_{1} q_{2}\right)\right) u$ that

$$
\left(q_{1} u\right)\left(q_{2} u\right)=\mu \bar{q}_{2} q_{1} \text {. }
$$

If $\mu=0, Q u$ is a nilpotent ideal in $C$. Hence we must assume also that $\mu \neq 0$. One verifies that the resulting algebra $C$ is alternative. We can extend the involution $q \rightarrow \bar{q}$ in $Q$ to one in $C$ in such a way that $\bar{u}=-u$. The self-adjoint elements relative to this involution are the elements of $\Phi(=\Phi 1)$. Hence we can write $C=C_{0}+\Phi 1$ where $\mathrm{C}_{0}$ is the set of skew elements. It is easy to see that $(x, y)=2(x y+y x)$ is a non-degenerate scalar product in $C_{0}$. Since the Jordan algebra $C_{J}$ is the Jordan algebra of this scalar product, $C_{J}$ is simple (Prop. 11.1). This implies that $C$ is a simple alternative algebra. It is easy to verify that the nucleus of $C$ is $\Phi 1$. Also the only elements which commute with all the elements of $C$ are those in $\Phi 1$.

In order to determine the finite dimensional simple Jordan algebras, one assumes first that the base field is algebraically closed. In this case one defines the degree of the algebra to be the maximum number of orthogonal idempotents in the algebra. At the present time it is not certain that one knows all the simple algebras of degree one. One might conjecture that the only algebra of this type is the one dimensional algebra. This is known to be the case for special Jordan algebras and for algebras of characteristic 0 .

It is easy to see that the simple algebras of degree two are Jordan algebras determined by non-degenerate symmetric scalar products. Suppose now that $\Im$ has degree at least three. Then $\Im$ has 
an identity and $1=\sum_{1}^{n} e_{i}, n \geqslant 3$, where the $e_{i}$ are orthogonal idempotents which are primitive in the sense that the terms $\mathfrak{I}_{i i}$ (cf eq. (26)) of the Peirce decomposition have no idempotents other than the $e_{i}$. If $\Im=\sum_{i \leq j} \oplus \mathfrak{\Im}_{i j}$ is the Peirce decomposition, then one can prove that, for every $i, j \neq, \mathfrak{\Im}_{i i}+\mathfrak{F}_{j j}+\mathfrak{\Im}_{i j}$ is the Jordan algebra of a symmetric non-degenerate scalar product. If follows that we can find a $u_{i j}$ such that $u_{i j}^{2}=e_{i}+e_{j}$. We can therefore apply the structure theorem, Th. 9.1 (cf. the remark following this theorem). Let $D$ be the alternative (or associative) algebra with involution furnished by this theorem. If $\mathfrak{D}$ has a radical $\mathfrak{S}$, then $\mathfrak{S}$ is a self-adjoint ideal. Then,

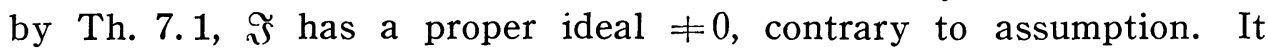
follows that $\mathfrak{D}$ is semi-simple so that $\mathscr{D}$ is a direct sum of simple algebras. In fact, it is now clear that either $\mathfrak{D}$ is simple or $\mathfrak{D}$ is a direct sum of two anti-isomorphic simple algebras. If we make use of the fact that the self-adjoint elements are in the nucleus and that the $e_{i}$ are primitive we see that the only possibilities for $D$ are the following :

A. $D$ is a direct sum of two one dimensional algebras. B. $\mathfrak{D}$ is one dimensional. C. $\mathcal{D}$ is a quaternion algebra $\left(=\Phi_{2}\right)$ with the involution whose self-adjoint elements are the elements of $\Phi$. E. $D$ is the Cayley algebra and the involution in $\mathfrak{D}$ is the one whose set of self-adjoint elements is $\Phi$. Accordingly, we speak of a simple Jordan algebra of type A, B, C or E. Of course, the last occurs only if $n=3$. The corresponding algebra is usually denoted as $M_{3}^{8}$. By Th. 8.1, this type of construction really does give a Jordan algebra and by Th. 8.2, this algebra is exceptional. The other simple Jordan algebras which we have listed are all special.

Suppose now that $\mathfrak{\Im}$ is a central simple Jordan algebra over an arbitrary base field $\Phi$. Then if $\Omega$ is the algebraic closure of $\Phi, \Im_{\Omega}$ is simple and hence is one of the algebras which we have listed. If $\mathfrak{\Im}_{\Omega}$ is one dimensional then so is $\Im$. If $\mathfrak{\Im}_{\Omega}$ is of degree two then it is easy to see that $\mathfrak{I}$ is the algebra of a non-degenerate symmetric scalar product. If $\mathfrak{\Im}_{\Omega}$ is of degree at least three and is special, then it is the set of self-adjoint elements of a matrix algebra $\mathfrak{D}_{n}(n$ the degree) with a canonical involution. Hence, by Th. 12.1, § itself is the set of self-adjoint elements of an associative algebra $\mathfrak{A}$ with an involution. One sees easily that $\mathfrak{A}$ is either simple or a direct sum of two anti-isomorphic simple algebras. ${ }^{22)}$ If $\mathfrak{J}_{\Omega}$ is $M_{3}^{8}$ then it has

22) These results are due to Kalisch [21] and to F. D. and N. Jacobson [15]. 
been shown by Schafer [31] that $\Im$ is the set of self-adjoint elements of $C_{3}$ where $C$ is a Cayley algebra and the involution is canonical in $C_{3}$ and is based on the usual involution in $C$.

\section{Representation theory for the exceptional cases}

In this section we shall determine the birepresentations of the exceptional simple alternative algebras $C$ and the exceptional simple Jordan algebras $M_{3}^{8}$.

Theorem 18.1. Let $C$ be a Cayley algebra over an arbitrary field $\Phi$ and let $\mathfrak{M}$ be an alternative bimodule for $C$. Then $\mathfrak{M}=\mathfrak{M}_{1} \oplus \mathfrak{M}_{0}$ where $\mathfrak{M}_{1}$ is a unital sub-bimodule and $\mathfrak{M}_{0}$ is the sub-bimodule annihilated on both sides by C. Any unital sub-bimodule is a direct sum of sub-bimodules which are isomorphic to $C$.

Proof. We have the Peirce decomposition $\mathfrak{M}=\mathfrak{M}_{00} \oplus \mathfrak{M}_{01} \oplus \mathfrak{M}_{10}$ $\oplus \mathfrak{M}_{11}$ relative to the identity of $C$. Now the sub-bimodules $\mathfrak{M}_{01}$ and $\mathfrak{M}_{10}$ are associative. Since $C$ is simple and not associative, it follows that these are 0 . Hence if we set $\mathfrak{M}_{1}=\mathfrak{M}_{11}, \mathfrak{M}_{0}=\mathfrak{M}_{00}$, we obtain the first statement. We now assume that $\mathfrak{M}=\mathfrak{M}_{1}$ is unital. Let $a \rightarrow L_{a}, a \rightarrow R_{a}$ denote the birepresentation and let $\mathfrak{R}_{1}$ and $\mathfrak{L}_{2}$ denote the Lie algebras of linear transformations generated by $\left\{L_{a}\right\}$ and $\left\{R_{a}\right\}$ respectively. Then $\mathfrak{R}_{1}=\Phi 1+\mathfrak{R}_{1}^{\prime}$ where $\mathfrak{R}_{1}^{\prime}$ is the ideal generated by the $\mathscr{R}_{a}$ with $a$ in $C_{0}$, the set of skew elements relative to the usual involution in $C$. Similarly $\mathfrak{\Omega}_{2}=\Phi 1+\mathfrak{\Omega}_{2}{ }^{\prime}$. Let $\&$ denote the Lie algebra generated by the $L_{a}$ and the $R_{a}$. Since we can deduce from (9) that

$$
\begin{aligned}
{\left[L_{a} R_{b}\right] } & =\frac{1}{2}\left(R_{a b-b a}-\left[R_{a} R_{b}\right]\right) \\
& =\frac{1}{2}\left(L_{b a-a b}-\left[L_{a} L_{b}\right]\right)
\end{aligned}
$$

we see that $\mathfrak{\Omega}_{1}{ }^{\prime}, \mathfrak{R}_{2}{ }^{\prime}$ are ideals in $\mathfrak{R}$. We observe next that $a \rightarrow L_{a}$, $a \rightarrow R_{a}$ define special representations of the Jordan algebra $C_{J}$ and we recall that this is the Jordan algebra of a non-degenerate scalar product in $C_{0}$. Hence by the theory of Clifford algebras, these representations are completely reducible. Assume, for the moment, that $\mathfrak{M}=C$ and consider the two representation $a \rightarrow L_{a}, a \rightarrow R_{a}$ of $C_{J}$. We assert that these are not equivalent. Otherwise, we have a 1-1 transformation $S$ in $C$ such that $R_{a}=S^{-1} L_{a} S$, that is, $(x S) a=(a x) S$ holds for all $a, x$ in $C$. Then $(a x) S^{2}=(a S)(x S)$. If we set $a=1$ this gives $(1 S)(x S)=(x S)(1 S)$. Hence $1 S=\sigma 1, \sigma \in \Phi$. We may now nor- 
malize $S$ so that $1 S=1$. Then $x S^{2}=x S$ or $S^{2}=S$. This implies that $S=1$. Thus we have $L_{a}=R_{a}$ which is clearly impossible. We therefore see that the Clifford algebra $\mathfrak{u}_{s}\left(C_{J}\right)$ has two inequivalent 8 dimensional unital representations. Thus $\mathfrak{U}_{s}\left(C_{J}\right)$ is a direct sum of two central simple algebras $\Delta_{k}$ where $\Delta$ is a central division algebra of $h^{2}$ dimensions and $h k=8$. The irreducible representations of this algebra are realized in a space of $h^{2} k$ dimensions. Hence $h^{2} k \leqslant h k$ and so $h=1, k=8, \Delta=\Phi$. Hence the irreducible representations of $\mathfrak{u}_{s}\left(C_{J}\right)$ are in 8 dimensional spaces and consequently $a \rightarrow L_{a}, a \rightarrow R_{a}$ give the two inequivalent irreducible representations. We recall also that, by Prop. 13.3, the Lie algebra generated by the mappings $\bar{R}_{a}=L_{a}+R_{a}, a \in C_{0}$, is simple. We have the relation

$$
\left[\left[\bar{R}_{a} \bar{R}_{b}\right] \bar{R}_{c}\right]=\bar{R}_{a}, \quad d=\{\{b c\} a\}-\{b\{c a\}\}
$$

for $a, b, c$ in $C_{0}$. We return now to the general unital bimodule $M$. Since $a \rightarrow L_{a}, a \rightarrow R_{a}$ are special representations of $C_{J}, L_{a} \neq 0$ and $R_{b} \neq 0$ for some $a, b \in C_{0}$. Otherwise, we should have a unital representation of the Clifford algebra $\mathfrak{u}_{s}\left(C_{J}\right)$ in a one dimensional space. Thus $\mathfrak{L}_{1}^{\prime}$ and $\mathfrak{R}_{2}^{\prime}$ are $\neq 0$. By (90) and Prop. 13.3, $\bar{R}_{a} \rightarrow L_{a}$ and $\bar{R}_{a} \rightarrow R_{a}$ determine isomorphisms. Hence $\mathfrak{L}_{1}^{\prime}$ and $\mathfrak{L}_{2}^{\prime}$ are simple Lie algebras. Since these are ideals in the Lie algebra $\mathscr{Q}$ so is their intersection. Hence either $\mathfrak{R}_{1}^{\prime}=\mathfrak{R}_{2}^{\prime}$ or $\mathfrak{R}_{1}^{\prime} \cap \mathfrak{R}_{2}^{\prime}=0$. In the latter case $\left[L_{a} R_{b}\right]=0$ for all $a$ and $b$. Thus the birepresentation is associative and we have a contradiction. We have therefore proved that $\mathfrak{R}_{1}^{\prime}=\mathfrak{R}_{2}^{\prime}, \mathfrak{R}_{1}=\mathfrak{R}_{2}$. Now we know that the set $\mathfrak{R}_{1}$ is completely reducible since $a \rightarrow L_{a}$ is a special representation of $C_{J}$. Hence our bimodule is completely reducible and its irreducible sub-bimodules are just the irreducible invariant subspaces relative to the $L_{a}$. We suppose finally that $\mathfrak{M}$ is an irreducible unital bimodule. Then our reasoning shows that it is 8 dimensional and $a \rightarrow L_{a}, a \rightarrow R_{a}$ are irreducible special representations of $C_{J}$. They are therefore equivalent to one of the representations $a \rightarrow L_{a}, a \rightarrow R_{a}$ acting in $C$. Hence we may as well identify the space $\mathfrak{M}$ with $C$ and we may take $L_{a}=l_{a}$ or $L_{a}=r_{a}$ where $l_{a}, r_{a}$ now denote the multiplications in $C$. Assume first that we have $L_{a}=l_{a}$. Then, by $(90),\left[L_{a} R_{b}\right]=\left[L_{a} r_{b}\right]$ for all $a, b \in C_{0}$. Since $\mathscr{L}_{1}^{\prime}$ is simple and contains the $R_{b}$ and $r_{b}$ this implies that $R_{b}=r_{b}$. It follows that $\mathfrak{M}$ and $C$ are identical. Next assume that $L_{a}=r_{a}$. Then, by $(90), R_{b}$ satisfies

$$
\left[r_{a} R_{b}\right]=\frac{1}{2}\left(r_{b a-a b}-\left[r_{a} r_{b}\right]\right)
$$


On the other hand, we can deduce from (89) that

$$
\left[r_{a},-l_{b}-r_{b}\right]=\frac{1}{2}\left(r_{b a-a b}-\left[r_{a} r_{b}\right]\right) .
$$

It follows that $R_{b}=-\left(l_{b}+r_{b}\right)$ for all $b \in C_{0}$. Let $\{b b\}=\beta 1$. Then $\beta x=x R_{\{b b \mathrm{j}}=x\left\{R_{b} R_{b}\right\}=b^{2} x+x b^{2}+2 b x b=\beta x+2 b x b$. Hence $b x b=0$ for all $x$ and so $b^{2}=0$ for all $b \in C_{0}$. This contradicts the fact that the scalar product associated with $C_{0}$ is non-degenerate. Hence $L_{a}=r_{a}$ is impossible and the only irreducible bimodule is essentially $C$. This completes the proof. $^{23)}$

We shall apply the result we have just obtained to determine the modules for the exceptional simple Jordan algebras. To do this we shall consider the following question: If $\mathfrak{M}$ is a unital bimodule for $C$ in how many ways can one introduce an involution in $\mathfrak{M}$ so that the self-adjoint elements are in the nucleus $\mathfrak{N}$ of $\mathfrak{M}$ ? In order to determine $\mathfrak{N}$ we consider a direct decomposition of $\mathfrak{M}$ into irreducible sub-bimodules $\mathfrak{M}_{\alpha}$. Then $\mathfrak{N}$ is the sum of the nuclei of the $\mathfrak{M}_{\alpha}$. If $\mathfrak{R}_{\alpha}$ is the nucleus of $\mathfrak{M}_{\alpha}, \mathfrak{N}_{\alpha}$ corresponds to the nucleus $\Phi 1$ of $C$ under an isomorphism of $C$ onto $\mathfrak{M}_{\alpha}$. It follows that the elements $u$ of the nucleus can also be characterized by the property $a u=u a$ for all $a \in C$. Now suppose that we have an involution $m \rightarrow \bar{m}$ in $\mathfrak{M}$ so that $\mathfrak{M}$ becomes a $C$-bimodule with involution. Then $\mathfrak{N}$ is mapped into itself by this involution. If the induced mapping is not the identity then we have a non-zero element $u \in \mathfrak{N}$ such that $\bar{u}=-u$. Then if $a \in C_{0}, \overline{a u}=u a=a u$. Hence $a u \in \mathfrak{N}$. If we refer to the isomorphism of the $\mathfrak{M}_{\alpha}$ with $C$ we see that this is impossible for $a \neq 0$. Thus $\bar{u}=u$ for all $u \in \mathfrak{N}$. Hence $\overline{a u}=-a u$ for all $u \in \mathfrak{R}, a \in C_{0}$. This implies that the $\mathfrak{M}_{\alpha}$ are isomorphic with $C$ even as bimodules with involution and $\mathfrak{M}$ as bimodule with involution is a direct sum of copies of $C$.

Theorem 18.2. Let $\mathfrak{\Im}$ be an exceptional simple Jordan algebra (finte dimensional) and let $\mathfrak{M}$ be a Jordan module for $\mathfrak{s}$. Then $\mathfrak{M}$ is a direct sum of a unital submodule and a submodule annihilated by $\Im$. Any unital $\Im$-module is a direct sum of submodules isomorphic to $\Im$.

Proof. The first statement follows from the Peirce decomposition

23) The foregoing proof has the same basic idea as that of Theorem 11.1, namely, the subordination of the alternative theory to the Jordan thoory. It is possible to derive this result also by strictly alternative methods based on properties of the Peirce decomposition. Cf, Zorn [37], Albert [5]. 
and the fact that $\Im$ is simple and not special. We suppose now that $M$ is unital and, for the present, we assume that the involution in the matrix algebra over $C$ giving $\Im$ is standard. Then according to Th. 10.3, $\mathfrak{M}$ determines a unital $C$-bimodule $\mathfrak{P}$ with involution such that the self-adjoint elements of $\mathfrak{P}$ are in the nucleus. Hence $\mathfrak{S}$ is a direct sum of copies of $C$. This implies that $\mathfrak{M}$ is a direct sum of copies of $\Im$. This proves the theorem if the involution defining $\Im$ is standard. Also we see that in this case the universal associative algebra $\mathfrak{u}(\mathfrak{\Im})$ of the representations is simple, for its representations are completely reducible and there is just one irreducible representation. Now if $\Im$ is arbitrary then we can find an extension $P$ of the base field so that $\mathfrak{\Im}_{\mathrm{P}}$ is given by a standard involution. Then $\mathfrak{u}(\mathfrak{\Im})_{\mathbf{P}}=\mathfrak{U}\left(\mathfrak{S}_{\mathbf{P}}\right)$ is simple. Hence $\mathfrak{U}(\mathfrak{\Im})$ is simple. This implies that the representations of $\Im$ are completely reducible and that in the sense of isomorphisms we have only one irreducible representation. This is, of course, the regular representation. Hence the theorem holds for arbitrary \%.

\section{Representation of separable algebras}

A finite dimensional alternative or Jordan algebra is said to be separable if it is semi-simple and its center is a direct sum of separable fields. If $\mathfrak{A}$ is such an algebra and $\Omega$ is the algebraic closure of the base field, then $\mathfrak{A}_{\Omega}$ is semi-simple. In the preceding section we have determined the bimodules for the exceptional central simple alternative and Jordan algebras. We consider next another particular case of separable algebras, namely, the non-exceptional central simple algebras.

Theorem 19.1. Let $\mathfrak{A}$ be a central simple associative algebra of $n^{2}$ dimensions. Then every alternative bimodule for $\mathfrak{A}$ is completely reducible. If $n \neq 2$, every irreducible alternative bimodule is associative and there are three classes (in the sence of isomorphism) of irreducible bimodules. If $n=2$ then $\mathfrak{X}$ has four classes of irreducible alternative bimodules, three of which are associative and one a Cayley bimodule.

Proof. Suppose first the base field $\Phi$ is algebraically closed. Then $\mathfrak{U}=\Phi_{n}$. If $\mathfrak{M}$ is an alternative bimodule, then we have the Peirce decomposition $\mathfrak{M}=\mathfrak{M}_{00} \oplus \mathfrak{M}_{01} \oplus \mathfrak{M}_{10} \oplus \mathfrak{M}_{11} . \quad \mathfrak{M}_{00}$ is annihilated left and right by $\mathfrak{A} ; \mathfrak{M}_{01}\left(\mathfrak{M}_{10}\right)$ is an associative right (left) $\mathfrak{U}$-module and so is completely reducible. $\mathfrak{M}_{11}$ is unital. Hence if $n \neq 2$ this is associative and so it can be considered as a right 
module for the associative algebra $\Phi_{n} \times \Phi_{n}$. Hence it is completely reducible. If $n=2$ then $\mathfrak{M}_{11}$ is a direct sum of an associative bimodule and a Cayley bimodule (Th. 11.1). The associative part is completely reducible as before. Also it is clear from the definition of a Cayley bimodule that the lattice of sub-bimodules of a Cayley bimodule coincides with the lattice of sub-modules of the associative right module (or representation) used to construct the Cayley bimodule. Hence it is completely reducible. Since a simple associative algebra possesses just one irreducible right (left) module it is clear that if $n \neq 2$ then $\Phi_{n}$ has three classes of irreducible bimodules. If $n=2$ then any two Cayley bimodules are isomorphic if and only if the associative right modules used to define them are isomorphic. Hence in this case we have one additional class of irreducible bimodules. This proves the result for algebraically closed base fields. Also, in this case, we see that the universal associative algebra $\mathfrak{U}\left(\Phi_{n}\right)$ of the birepresentations is semi-simple and we can determine its structure. Thus each class of irreducible birepresentation accounts for a simple component of $\mathfrak{u}\left(\Phi_{n}\right)$. Hence if $n \neq 2$ then $\mathfrak{u}\left(\Phi_{n}\right)$ is a direct sum of three simple algebras and if $n=2$ then $\mathfrak{U}\left(\Phi_{n}\right)$ is a direct sum of four simple algebras. Now let the base field be arbitrary and let $\Omega$ be its algebraic closure. Then $\mathfrak{u}(\mathfrak{U})_{\Omega}=\mathfrak{u}\left(\mathfrak{A}_{\Omega}\right)=\mathfrak{U}\left(\Omega_{n}\right)$ since $\mathfrak{A}_{\Omega}=\Omega_{n}$. Hence $\mathfrak{u}(\mathfrak{A})$ is semi-simple and the bimodules are completely reducible. Now $\mathfrak{Z}$ has an irreducible associative right (left) module and an irreducible associative unital bimodule. Evidently no two of these are isomorphic. If $n=2$ we have also an irreducible Cayley bimodule. Hence $\mathfrak{U}(\mathfrak{U})$ is a direct sum of at least three simple components if $n \neq 2$ and of at least four simple components if $n=2$. Since $\mathfrak{u}(\mathfrak{A})_{\Omega}$ is a direct sum of three or four components in these two cases it is clear that $\mathfrak{u}(\mathfrak{U})$ is a direct sum of exactly three simple algebras if $n \neq 2$ and of exactly four simple algebras if $n=2$. Since these correspond to the irreducible bimodules we have indicated, the latter give a complete list of sub-bimodules. This completes the proof.

We note also that we can calculate the dimensionality of $\mathfrak{u}(\mathfrak{A})$. Thus the dimensionality of a simple component is the dimensionality of the enveloping associative algebra of the corresponding irreducible bimodules. Hence if $n \neq 2$ then $\operatorname{dim} \mathfrak{u}(\mathfrak{A})=n^{2}+n^{2}+n^{4}=n^{2}\left(n^{2}+2\right)$ and if $n=2$ then $\operatorname{dim} \mathfrak{u}(\mathfrak{U})=28$.

We shall say that a central simple Jordan algebra $\Im$ is of degree $k$ if $\Im_{\Omega}$ is of degree $k$ for $\Omega$ the algebraic closure of the base field.

PROPOSITION 19.1. Let $\Im$ be a special central simple Jordan algebra 
of degree $n \geqslant 3$. Then every Jordan module for $\Im$ is completely reducible.

Proof. The theory of Peirce decomposition reduces the considerations to special modules and unital modules. Since the universal associative algebra is either simple or a direct sum of two simple algebras, the special modules are completely reducible. We consider next the unital modules and we suppose at first that the base field is algebraically closed. Let $\mathfrak{M}$ be unital and let $\mathfrak{D}$ be the associative algebra associated with $\mathscr{D}$ in the structure theory $(\S 17)$, so that $\mathfrak{D}$ is either a direct sum of two algebras of $\operatorname{dim} 1$ (type A), is of $\operatorname{dim} 1$ (type B) or is the matrix algebra $\Phi_{2}$ (type C). The involution in $D$ in the first case exchanges the two component algebras and in the last it has the form $a \rightarrow \operatorname{tr}(a)-a$ so that the self-adjoint elements are the elements of $\Phi$. We introduce the algebra $\mathfrak{B}$, the cross product $(\mathfrak{D} \times \mathfrak{D}, P, 1)$ defined in $\S 10$. In the first case, $\mathbb{S}$ is a direct sum of a quaternion algebra and four one dimensional algebras. In the second, $\mathbb{B}$ is a direct sum of two algebras of order one and in the third $\$$ is a direct sum of two matrix algebras $\Phi_{4}$. These results are easily proved and we leave the details to the reader. If $n>3$ we can reduce the discussion of $\mathfrak{M}$ to that of an associative $\mathfrak{D}$ bimodule with involution and then to a right module for (B). Since (B) is semi-simple all of these are completely reducible. If $n=3$ we have to consider also alternative $\mathfrak{D}$-bimodules with involution such that the self-adjoint elements are in the nucleus. Now the alternative bimodules for the algebra $\mathfrak{D}$ we are considering are all associative except for $\mathscr{D}=\Phi_{2}$. In the associative cases (types A,B) we again get a reduction to right $(\mathbb{S}$-modules and complete reducibility holds. We have to consider finally alternative $\Phi_{2}$-bimodules with involutions. Hence let $\mathfrak{P}$ be a unital alternative bimodule for $\Phi_{2}$. Then $\mathfrak{P}=\mathfrak{P}_{1} \oplus \mathfrak{F}_{2}$ where $\mathfrak{F}_{1}$ is associative and $\mathfrak{F}_{2}$ is a Cayley bimodule. It is easy to see that the nucleus of $\mathfrak{F}_{2}$ is 0 . Hence $\mathfrak{F}_{1}$ is the nucleus of $\mathfrak{P}_{\text {. On }}$ the other hand, the only element $z_{1}$ in $\mathfrak{B}_{1}$ such that $a z_{1}=z_{1} \bar{a}$ for all $a \in \Phi_{2}$ is $z_{1}=0$. Hence $\mathfrak{P}_{2}$ can be characterized as the set of elements $z$ in $\mathfrak{P}$ such that $a z=z \bar{a}$ for all $a$. It now follows that if $\mathfrak{s}^{3}$ has an involution then both $\mathfrak{F}_{1}$ and $\mathfrak{P}_{2}$ are self-adjoint. Hence $\mathfrak{F}_{1}$ is an associative $\Phi_{2}$-bimodule with involution and is completely reducible since $(S)$ is semi-simple. Now in $\mathfrak{P}_{2}$ we require the self-adjoint elements to be in the nucleus. Since the nucleus is 0 the involution maps every element into its negative. Hence every sub-bimodule is self-adjoint. It follows that $\mathfrak{B}_{2}$ is completely reducible as bimodule 
with involution. Thus we see that complete reducibility holds also for type C, $n=3$. This completes the proof for algebraically base fields. As in the alternative case, the use of universal associative algebra of the representations permits us to extend the result to arbitrary base fields.

The proof of the foregoing theorem gives an enumeration of the classes of irreducible unital modules in the algebraically closed case. Thus the consideration of the structure of (S) shows (via Th. 10.3, $10.4)$ that if $\mathfrak{J}$ is of type A, there are five such classes and if $\mathfrak{J}$ is of type $\mathrm{B}$ or of type $\mathrm{C}$ with $n>3$ there are two. If $\Im$ is of type $\mathrm{C}$ with $n=3$ then there are three. Thus let $\mathfrak{s}$ be an irreducible Cayley bimodule for $\Phi_{2}$. Then we can make $\mathfrak{*}$ into a bimodule with an involution by defining $\bar{x}=-x$ for all $x \in{ }^{3}$. Then the self-adjoint elements of the split null extension $\mathfrak{F}=\Phi_{2} \oplus \mathfrak{P}$ are multiples of 1 . Hence they are in the center of $\mathfrak{\mho}$. It follows that $\mathfrak{s}$ determines an irreducible unital module for the simple Jordan algebra $\Im$ of type $\mathrm{C}$ with $n=3$. We remark also that the corresponding split null extension is a new exceptional Jordan algebra of 21 dimensions.

Using the information just obtained we can determine the number of classes of irreducible modules for special central simple algebras of degree $n \geqslant 3$ over an arbitrary base field. In several case we can determine quite explicitly the irreducible modules. In addition to the results in the algebraically closed case, we shall need to investigate the structure of the universal associative algebra $\mathfrak{M}_{s}^{(2)}(\Im)$.

We say that the Jordan algebra $\mathfrak{\Im}$ is of type $\mathrm{A}, \mathrm{B}, \mathrm{C}$ or $\mathrm{E}$ if $\mathfrak{\Im}_{\Omega}$ is of type $\mathrm{A}, \mathrm{B}, \mathrm{C}$ or $\mathrm{E}$ for $\Omega$ the algebraic closure of the base field.

It is known that any algebra of types $\mathrm{B}$ or $\mathrm{C}$ is isomorphic to the algebra $\mathfrak{S}$ of self-adjoint elements of a central simple associative algebra $\mathfrak{A}$ with an involution (of first kind). ${ }^{24}$ If the degree of $\mathfrak{S}$ is $n$, then $\operatorname{dim} \mathfrak{S}=n(n+1) / 2$ for type $\mathrm{B}$ and $\operatorname{dim} \mathfrak{S}=n(2 n-1)$ for type C. The universal associative algebra of $\mathfrak{S}$ is $\mathfrak{A}$ and the fundamental involution is the involution defining $\mathfrak{L}$. The dimensionality of $\mathfrak{A}$ is $n^{2}$ for type $\mathrm{B}$ and $4 n^{2}$ for type C. If $\mathfrak{\Im}$ is of type A either $\mathfrak{\Im}$ is isomorphic to an algebra $\mathfrak{U}_{J}, \mathfrak{A}$ central simple associative or $\mathfrak{\Im}$ is isomorphic to the algebra $\mathfrak{S}$ of self-adjoint elements of a simple associative algebra $\mathfrak{A}$ with an involution of second kind. Accordingly, we say that $\mathfrak{J}$ is of type $A_{I}$ or type $A_{I I}$. The universal associative algebra of $\mathfrak{\Im}=\mathfrak{A}_{J}$ of type $A_{I}$ is the direct sum $\mathfrak{A} \oplus \mathfrak{X}^{\prime}$ where $\mathfrak{X}^{\prime}$ is

24) The results stated without proof in this section can be found in F. D. and $N$. Jacobson [15]. 
anti-isomorphic to $\mathfrak{A}$. The universal associative algebra of $\mathfrak{\Im}=\mathfrak{S}$ of type $A_{I I}$ is $\mathfrak{A}$, the fundamental involution is the given one. For central simple Jorden algebras of type $\mathrm{A}$ and degree $n$ we have $\operatorname{dim} \mathfrak{\Im}=n^{2}$ and $\operatorname{dim} \mathfrak{U}_{s}(\mathfrak{\Im})=2 n^{2}$. Coversely, the constructions we have indicated here always yield central simple Jordan algebras of type $\mathrm{A}, \mathrm{B}$ or $\mathrm{C}$.

Proposition 19.2. Let $\mathfrak{A}$ be a finite dimensional central simple associative algebra with an involution and let $\mathfrak{S}$ denote the set of self-adjoint elements, $\mathfrak{S}$ the set of skew elements. Assume deg $\mathfrak{E} \geqslant 3$. (1) Then the subalgebra $\mathfrak{H}_{s}^{(2)}(\mathfrak{S})$ of $\mathfrak{A} \otimes \mathfrak{A}$ generated by the elements $h \otimes 1+1 \otimes h, h \in \mathfrak{W}$ coicides with the set $\mathfrak{B}$ of elements invariant under the exchange automorphism $P$. (2) $\mathfrak{H}_{s}^{(2)} \cong \Phi_{r(r-1) / 2} \oplus \Phi_{r(r+1) / 2}$ if $\operatorname{dim} \mathfrak{A}=r^{2} \quad(3) \mathfrak{S}$ and $\subseteq$ are non-isomorphic irreducible unital modules for $\mathfrak{S}$.

Proof. (1) Assume first that the base field $\Phi$ is algebraically closed. Then $\mathfrak{A}=\mathfrak{D}_{n}$ where either $\mathfrak{D}=\Phi$ or $\mathfrak{D}=\Phi_{2}$ with involution $a \rightarrow \operatorname{tr} a-a$. In either case Prop. 7.3 shows that $\subseteq=$ [SES]. Hence, by Th. $10.3, \mathfrak{U}_{s}^{(2)}(\mathfrak{S})=\mathfrak{B}$. The usual extension of the base field argument shows that this holds for arbitrary base field $\Phi$. (2) By (1) $\operatorname{dim} \mathfrak{H}_{s}^{(2)}=r^{2}\left(r^{2}+1\right) / 2$. Since $\mathfrak{A}$ is central simple, the mappings $x \rightarrow\{x h\}, x \in \mathfrak{A}, h \in \mathfrak{S}$ determines a faithful representation of $\mathfrak{u}_{s}^{(2)}$. Evidently $\mathfrak{S}$ and $\subseteq$ are invariant subspaces for this representation. Their dimensionalities are $r(r+1) / 2, r(r-1) / 2$, respectively, for type $\mathrm{B}$ and $r(r-1) / 2, r(r+1) / 2$ for type $\mathrm{C}$. Accordingly the sum of the squares of these dimensionalities is $\operatorname{dim} \mathfrak{H}_{s}^{(2)}$. It follows that the induced set of linear transformations in $\mathfrak{S}$ and $\subseteq$ are the complete sets in these spaces. Hence $\mathfrak{u}_{s}^{(2)} \cong \Phi_{r(r-1) / 2} \oplus \Phi_{r(r+1) / 2}$ (cf. the proof of Th. 16.1). (3) This is an immediate consequence of (2).

PROPOSITION 19.3. Let $\mathfrak{A}$ be a central simple associative algebra of $n^{2}>4$ dimensions and let $\mathfrak{B}$ denote the subalgebra of $\mathfrak{A} \otimes \mathfrak{A}$ of elements invariant under the automorphism $P$. Then $\mathfrak{B}$ is a direct sum of two central simple algebras of dimensionalities $n^{2}(n+1)^{2} / 4$ and and $n^{2}(n-1)^{2} / 4$.

Proof. If the base field is algebraically closed $\mathfrak{A}=\Phi_{n}$ has an involution which gives an algebra of type $\mathrm{B}$ and degree $\geqslant 3$. Hence Prop. 19.2 (2) shows that $\mathfrak{B}=\Phi_{n(n-1) / 2} \oplus \Phi_{n(n+1) / 2}$. If $\Phi$ is arbitrary and $\Omega$ is its algebraic closure, then $\mathfrak{B}_{\Omega}=\Omega_{n(n-1) / 2} \oplus \Omega_{n(n+1) / 2}$. Hence either $\mathfrak{B}$ is simple or it is a direct sum of two simple algebras. On the other hand, it is well known that if a simple algebra remains 
semi-simple on extension of the base field to its algebraic closure, then the extended algebra is a direct sum of isomorphic simple algebras. ${ }^{25}$ Hence $\mathfrak{B}$ is a direct sum of two simple algebras. These become complete matrix algebras on extension of the base. Hence they are central. Also it is clear that their dimensionalities are as indicated.

Proposition 19.4. Let $\mathfrak{A}, \mathfrak{S}$ and $\subseteq$ be as in Prop. 19.2 and let $a \rightarrow \bar{a}$ be the involution in $\mathfrak{A}$. (1) Then the linear transformations

$$
\begin{array}{lll}
x \rightarrow\{x a\}, & x \in \mathfrak{N}, & a \in \mathfrak{A} \\
x \rightarrow x a+\bar{a} x, & x \in \mathfrak{S}, & a \in \mathfrak{A} \\
x \rightarrow x a+\bar{a} x, & x \in \mathfrak{S}, & a \in \mathfrak{A} \\
x \rightarrow a x+x \bar{a}, & x \in \mathfrak{S}, & a \in \mathfrak{A} \\
x \rightarrow a x+x \bar{a}, & x \in \mathfrak{S}, & a \in \mathfrak{A}
\end{array}
$$

give the five irreducible unital representations of $\mathfrak{A}_{J}$. (2) The algebra $\mathfrak{U}_{s}^{(2)}(\mathfrak{U})$ coincides with the subalgebra $\mathfrak{B}$ of $\mathfrak{U}_{s}(\mathfrak{H}) \otimes \mathfrak{U}_{s}(\mathfrak{H})$ of elements invariant under $P$.

Proof (1). We represent $\mathfrak{A}_{J}$ as the subset of matrices $A=\left(\begin{array}{ll}a & 0 \\ 0 & \bar{a}\end{array}\right)$ in the matrix algebra $\mathfrak{A}_{2}$. Then $X \rightarrow\{X A\}, X \in \mathfrak{A}_{2}$ defines a faithful representation of $\mathfrak{u}_{s}^{(2)}(\mathfrak{A})$. We can decompose $\mathfrak{A}_{2}$ as a direct sum of six invariant subspaces whose matrices are, respectively,

$$
\left(\begin{array}{ll}
a & 0 \\
0 & 0
\end{array}\right),\left(\begin{array}{ll}
0 & 0 \\
0 & a
\end{array}\right),\left(\begin{array}{ll}
0 & h \\
0 & 0
\end{array}\right),\left(\begin{array}{ll}
0 & s \\
0 & 0
\end{array}\right),\left(\begin{array}{ll}
0 & 0 \\
h & 0
\end{array}\right),\left(\begin{array}{ll}
0 & 0 \\
s & 0
\end{array}\right)
$$

where $a \in \mathfrak{A}, h \in \mathfrak{E}, s \in \mathfrak{S}$. The first and second are isomorphic and irreducible, since $\mathfrak{U}_{J}$ is simple. Hence we drop the second. The first and last four are isomorphic to the modules defined by (92). If we restrict $a$ in (92) to belong to $\mathfrak{S}$ then the last four modules become the irreducible $\mathfrak{S}$-modules considered in Prop. $19.2(3)$. Hence these are also irreducible for $\mathfrak{A}$. Since our representation of $\mathfrak{U}_{s}^{(2)}(\mathfrak{A})$ in $\mathfrak{A}_{2}$ is faithful and since $\mathfrak{U}_{s}^{(2)}(\mathfrak{U})$ is semi-simple (Prop. 19.1), the five irreducible modules we have displayed include all the irreducible unital modules for $\mathfrak{U}_{s}^{(2)}$. Now if the base field is algebraically closed, then we have seen that there are five non-isomorphic unital modules for $\mathfrak{U}_{s}^{(2)}$. Hence the modules we have defined are not isomorphic in this case. Clearly, this implies that they are not isomorphic for any base field. (2). We can calculate $\operatorname{dim} \mathfrak{U}_{s}^{(2)}$ as the sum of the squares

25) See, for example, Jacobson, Theory of Rings, p. 115. 
of the dimensionalities of the five irreducible modules. Comparison with $\operatorname{dim} \mathfrak{B}$ proves that $\mathfrak{U}_{s}^{(2)}(\mathfrak{A})=\mathfrak{B}$.

The simplest class of examples of simple associative algebras with involutions of second kind are the matrix algebras $\mathrm{P}_{n}$ where $\mathrm{P}$ is a quadratic extension of the base field $\Phi$. Here we take the involution $a \rightarrow \bar{a}^{\prime}$ where the ' denotes the transpose and $\bar{a}$ is the matrix obtained from $a$ by taking the conjugation in $\mathrm{P}$ of the elements of $a$. We observe that $a \rightarrow a^{\prime}$ and $a \rightarrow \bar{a}$ commute. We shall now consider a class of algebras with involutions of second kind which is somewhat more general than that of the algebras $P_{n}$.

PROPOSITION $_{\mathrm{N}}$ 19.5. Let $\mathfrak{A}$ be a simple associative algebra of dimensionality $2 n^{2}, n \geqslant 3$, with center $\mathrm{P}$ a quadratic extension of the base field. Suppose that $\mathfrak{A}$ has an involution $a \rightarrow a^{\prime}$ of first kind and an automorphism $a \rightarrow \bar{a}$ of period two which commutes with the given involution and which induces an automorphism $\neq 1$ in $P$. Then $a \rightarrow \bar{a}^{\prime}$ is an involution of second kind. Let $\mathfrak{S}$ be the set of selfadjoint elements of this involution. Then the linear transformations

$$
\begin{aligned}
& x \rightarrow\{x h\}, \quad x \in \mathfrak{S}, \quad h \in \mathfrak{Y} \\
& x \rightarrow x \bar{h}+h x, \quad x^{\prime}=x, \quad h \in \mathfrak{S} \\
& x \rightarrow x \bar{h}+h x, \quad x^{\prime}=-x, \quad h \in \mathfrak{E}
\end{aligned}
$$

define three non-isomorphic irreducible unital modules for $\mathfrak{g}$. Every irreducible unital $\mathfrak{2}$-module is isomorphic to one of these.

Proof. We use the automorphism $\sigma: a \rightarrow \bar{a}$ to define the crossed product $\mathfrak{F}=(\mathfrak{A}, \sigma, 1)$. Let $c$ be an element which we adjoin to $\mathfrak{A}$ to form $\mathfrak{F}$, so that $c a=\bar{a} c, c^{2}=1$. One verifies that $\mathfrak{F}$ is central simple. Hence $x \rightarrow\{x h\}, x \in \mathfrak{F}, h \in \mathfrak{S}$ defines $a 1-1$ representation of the semisimple associative algebra $\mathfrak{H}_{s}^{(2)}(\mathfrak{S})$. We have four invariant subspaces whose elements are, respectively, $h \in \mathfrak{S}, s$ skew $\left(s^{\prime}=-s\right), x c$ with $x^{\prime}=x$ and $x c$ with $x^{\prime}=-x$. There exists an element $\mu$ in $\mathrm{P}$ such that $\bar{\mu}=-\mu$. Multiplication by this element gives an isomorphism between the first two of our spaces. The sum of the squares of the dimensionalities of one of these and the last two is $n^{4}+n^{2}(n+1)^{2} / 2$ $+n^{2}(n-1)^{2} / 2=n^{2}\left(2 n^{2}+1\right)$. It follows from Prop. $19.4(2)$ that this equals $\operatorname{dim} \mathfrak{H}_{s}^{(2)}(\mathfrak{S})$. The proof can now be completed along the lines which we have used in Prop. 19.4.

We can now prove the following result.

Theorem 19.2. Let $\Im$ be any special central simple Jordan algebra of degree $n \geqslant 3$ over an arbitrary base field. Then the associative algebra 
$\mathfrak{u}(\mathfrak{I})$ is semi-simple and we have the following table on its structure

$\begin{array}{lcc}\text { type } & \text { dims. of simple components of } \mathfrak{U}(\mathfrak{\Im}) & \operatorname{dim} \mathfrak{u}(\mathfrak{\Im}) \\ \mathrm{A}_{\mathrm{I}} & n^{2}, n^{2}, n^{4}, n^{2}(n-1)^{2} / 4, n^{2}(n-1)^{2} / 4, n^{2}(n+1)^{2} / 4, n^{2}(n+1)^{2} / 4 & n^{2}\left(2 n^{2}+3\right) \\ \mathrm{A}_{\mathrm{II}} & 2 n^{2}, n^{4}, n^{2}(n-1)^{2} / 2, n^{2}(n+1)^{2} / 2 & n^{2}\left(2 n^{2}+3\right) \\ \mathrm{B} & n^{2}, n^{2}(n-1)^{2} / 4, n^{2}(n+1)^{2} / 4 & n^{2}\left(n^{2}+3\right) / 2 \\ \mathrm{C}, n>3 & 4 n^{2}, n^{2}(2 n-1)^{2}, n^{2}(2 n+1)^{2} & 2 n^{2}\left(4 n^{2}+3\right) \\ \mathrm{C}, n=3 & 36,225,441,36 & 738\end{array}$

Proof. The semi-simplicity of $\mathfrak{u}(\mathfrak{I})$ is a consequence of Prop. 17.1. If $\Im$ is of type $\mathfrak{A}$ then the extension of the base field argument and Prop. 17.4 (2) show that $\mathfrak{U}_{s}^{(2)}(\mathfrak{F})$ is the subalgebra $\mathfrak{B}$ of $\mathfrak{u}_{s}(\mathfrak{\Im}) \otimes \mathfrak{U}_{s}(\mathfrak{\Im})$ of elements invariant under $P$. If $\mathfrak{\Im}$ is of type $\mathrm{A}_{\mathrm{I}}$, $\mathfrak{\Im}=\mathfrak{A}_{J}, \mathfrak{A}$ central simple and $\mathfrak{U}_{\boldsymbol{s}}(\mathfrak{\Im})=\mathfrak{U} \oplus \mathfrak{U}^{\prime}$ where $\mathfrak{U}^{\prime}$ is anti-isomorphic to $\mathfrak{U}$. Then $\mathfrak{U}_{s} \otimes \mathfrak{U}_{s}=(\mathfrak{H} \otimes \mathfrak{U}) \oplus\left(\mathfrak{A} \otimes \mathfrak{U}^{\prime}\right) \oplus\left(\mathfrak{U}^{\prime} \otimes \mathfrak{U}\right) \oplus\left(\mathfrak{U}^{\prime} \otimes \mathfrak{U}^{\prime}\right)$ and $P$ exchanges $\mathfrak{A} \otimes \mathfrak{U}^{\prime}$ and $\mathfrak{U}^{\prime} \otimes \mathfrak{U}$ and induces in $\mathfrak{U} \otimes \mathfrak{U}$ and $\mathfrak{U}^{\prime} \otimes \mathfrak{U}^{\prime}$ the same kind of exchange mapping. Now $\mathfrak{A} \otimes \mathfrak{H}^{\prime} \simeq \Phi_{n^{2}}$ and by Prop. 17. 3, $\mathfrak{B} \cap(\mathfrak{U} \otimes \mathfrak{U})$ and $\mathfrak{B} \cap\left(\mathfrak{U}^{\prime} \otimes \mathfrak{U}^{\prime}\right)$ are direct sums of central simple algebras of dimensionalities $n^{2}(n+1)^{2} / 4$ and $n^{2}(n-1)^{2} / 4$. Hence we have proved everything for type $A_{I}$. Next let $\Im$ be of type $A_{I I}$ so that $\mathfrak{F}=\mathfrak{S}$ the set of self-adjoint element in the simple algebra $\mathfrak{U}$. with an involution of second kind. Then $\mathfrak{U}_{\boldsymbol{s}}(\mathfrak{\Im})=\mathfrak{A}$ has center which is a quadratic extension of the base field. Hence $\mathfrak{U} \otimes \mathfrak{U}$ $=\mathfrak{U}_{1} \oplus \mathfrak{A}_{2}$ where $\mathfrak{A}_{i}$ is simple of $n^{4}$ dimensions over its center $\mathrm{P}_{\boldsymbol{i}}$ which is two dimensional. Let $\mathfrak{B}$ be the subalgebra defined before. If we extend the base field $\Phi$ to its algebraic closure $\Omega$, then the preceding discussion shows that $\mathfrak{B}_{\Omega}$ is a direct sum of five simple algebras and that, for the center $P_{1} \oplus \mathbf{P}_{2}$, we have $\operatorname{dim}\left(\left(\mathbf{P}_{1} \oplus \mathbf{P}_{2}\right)_{\Omega} \cap\right.$ $\left.\mathfrak{B}_{\Omega}\right)=3$. These two facts imply that $\mathrm{P}$ maps each $\mathfrak{A}_{i}$ into itself, induces the identity mapping in one of the $P_{i}$, say $P_{1}$, and induces an automorphism of period two in the other. It follows that $\mathfrak{B} \cap \mathfrak{A}_{2}$ is a central simple algebra of $n^{4}$ dimensions. Hence $\left(\mathfrak{B} \cap \mathfrak{U}_{1}\right)_{\Omega}$ is a direct sum of two simple algebras of $n^{2}(n+1)^{2} / 4$ dimensions and two of $n^{2}(n-1)^{2} / 4$ dimensions. It is easy to see that this can happen only if $\mathfrak{B} \cap \mathfrak{U}_{1}$ is a direct sum of two simple algebras having centers which are quadratic fields and having dimensionalities $n^{2}(n+1)^{2} / 4$ and $n^{2}(n-1)^{2} / 4$ over these. This proves the assertions about $\Im$ of type $\mathrm{A}_{\mathrm{II}}$. If $\Im$ is of type $\mathrm{B}$ or $\mathrm{C}, n>3$ then the results follow from Prop. 19.1, 19.2 and the fact that the universal associative algebra $\mathfrak{u}_{s}(\mathfrak{\Im})$ is simple. We consider finally type C, $n=3$. If the base field is algebraically closed then we have seen that there exists an irreducible module which is not a submodule of a sum of two commuting 
special modules. The dimensionality of the corresponding simple components of $\mathfrak{u}(\mathfrak{I})$ is 36 . The usual argument with universal assocrative algebras shows that this is valid also for arbitrary base field.

The results which we have obtained for the various types of central algebras can be put together to obtain the irreducible bimodules for separable alternative and Jordan algebras. We shall not attempt to make this explicit but shall be content to derive the following fundamental result.

Theorem 19.3. If $\mathfrak{A}$ is a separable alternative algebra or a known separable Jordan algebra of finite dimensions, then every bimodule for $\mathfrak{A}$ is completely reducible.

Proof. It suffices to prove this for algebraically closed base fields. Write $\mathfrak{A}=\mathfrak{A}_{1} \oplus \mathfrak{A}_{2} \oplus \cdots \oplus \mathfrak{A}_{r}$ where the $\mathfrak{A}_{i}$ are simple. If $\mathfrak{A}$ is alternative then we know that $\mathfrak{A}_{i}=\Phi_{n_{i}}$ or $=C$ the Cayley algebra. The theorem is therefore an immediate consequence of the Peirce decompositions, Ths. 11.1 and 19.1. Now suppose that $\mathfrak{A}$ is Jordan. Since we are assuming that the structure of $\mathfrak{A}$ is known, the $\mathfrak{A}_{i}$ are either one dimensional or have degrees $\geqslant 2$. The Peirce decompositions reduce the considerations to three cases: (1) special $\mathfrak{A}_{i}$-modules, (2) unital modules which are sums of two commuting special modules, one of which is an $\mathfrak{A}_{i}$-module and the other an $\mathfrak{A}_{3}$-module, $i \neq j$ and (3) unital $\mathfrak{A}_{i}$-modules. The result is clear for (1). In case (2), $\mathfrak{M}$ defines a representation of $\mathfrak{u}_{s}\left(\mathfrak{A}_{i}\right) \otimes \mathfrak{U}_{s}\left(\mathfrak{A}_{\mathfrak{j}}\right)$. Since this algebra is semi-simple, $\mathfrak{M}$ is completely reducible. In $(3)$, complete reducibility is trivial for one dimensional algebras. It follows for algebras of degree 2 from Th. 16.1 and 16.2 and for algebras of degree $\geqslant 3$ from Th. 18.2 and Prop. 19.1. This completes the proof.

Yale University

(Received December 17, 1953)

\section{Bibliography}

1. A. A. Albert, On a certain algebra of quantum mechanics, Ann. of Math., $\mathbf{3 5}$ (1934), 65-73.

2. - - A structure theory for Jordan algebras, Ann. of Math., 48 (1947), 446-467.

3. - A note on the exceptional Jordan algebra, Proc. Nat. Acad. Sci., 36 (1950), 372-374.

4. - - A theory of power associative commutative algebras, Trans. Amer. Math Soc., 69 (1950), 503-527.

5. _ - On simple alternative rings, Can. J. of Math., 4 (1952), 129-135.

6. A. S. Amitsur, On the identities of PI rings, Proc. Amer. Math. Soc,. 4, (1953), 27-35. 
7. G. Ancochea, On semi-automorphisms of division algebras, Ann. of Math., 48 (1947), 147-154.

8. G. Birkhoff, Representability of Lie algebras, etc.. Ann. of Math., 38 (1937), 326-332.

9. G. Birkhoff and P. Whitman, Representations of Jordan and Lie algebras, Trans. Amer. Math. Soc., 61 (1949), 116-136.

10. R. Brauer and H. Weyl, Spinors in n dimensions, Amer. J. of Math., 57 (1935), 425-449.

11. C. Chevalley and R. D. Schafer, The exceptional simple Lie algebras $\widetilde{F}_{4}$ and $\mathfrak{F}_{6}$, Proc. Nat. Acad. Sci., 36 (1950), 137-141.

12. R. J. Duffin, On the characteristic matrices of covariant systems, Phys. Rev., 54 (1938), 1114.

13. S. Eilenberg, Extensions of general algebras, Annales de la Société Polonaise de Mathématique, 21 (1948), 125-34.

14. H. Freudenthal, Oktaven, Ausnahmengruppen und Oktavengeometrie, Utrecht 1951.

15. F. O. Jacobson and N. Jacobson, Classification and representation of semi-simple Jordan algebras, Trans. Amer. Math. Soc. 65 (1949), 141-169.

16. N. Jacobson, Cayley numbers and simple Lie algebras of type G, Duke Math. I., 5 (1939), 775-783.

17. - Classes of restricted Lie algebras of characteristic p, I, Amer. J. of Math. 63 (1941), 481-515.

18. - Isomorphisms of Jordan rings, Amer. J. of Math., 70 (1948), 317-326.

19. - Derivation algebras and multiplication algebras of semi-simple Jordan algebras, Ann. of Math., 50 (1949), 866-874.

20. - , Lie and Jordan triple systems, Amer. J. of Math., 17 (1949), 149-170.

21. - General representation theory of Jordan algebras, Trans. Amer Math. Soc., 70 (1951), 509-530.

22. N. Jacobson and C. E. Rickart, Jordan Homomorphism of Rings, Trans. Amer. Math. Soc., 69 (1950), 479-502.

23. - - Homomorphisms of Jordan rings of selfadjoint elements. Trans. Amer. Math. Soc., 72 (1952), 310-322.

24. P. Jordan, Über die Multiplikation quanten-mechanischer Grössen, Zeitschrift für Physik, 80 (1933), 285-291.

25. P. Jordan, J. von Neumann and E. Wigner, On an algebraic generalization of the quantum mechanical formalism, Ann. of Math., 35 (1934), $29-64$.

26. G. K. Kalisch, On special Jordan algebras, Trans. Amer. Math. Soc., 61 (1947), 482-494.

27. I. Kaplansky, Semi-automorphisms of rings, Duke Math. J. 14 (1947), 521-527.

28. W. H. Mills, A theorem on the represention theory of Jordan algebras, Pacific J. of Math., 1 (1951), 255-264.

29. R. Moufang, Zur Struktur von alternativen Körpern, Math. Anna. 110 (1937), 416-430.

30. A. J. Penico, The Wedderburn principal theorem for Jordan algebras, Trans. Amer. Math. Soc., 70 (1951), 404-421.

31. R. D. Schafer, The exceptional simple Jordan algebras, Amer. J. of Math., 70 (1948), 82-94. 
32. — - Representations of alternative algebras, Trans. Amer.Math. Soc., 72 (1952), $1-17$.

33. W. Specht, Gesetze in Ringen I, Math Zeitschr. 52, (1950), 557-589.

34. N. Svartholm, On the algebras of relativistic quantum mechanics, Proc. of the Royal Physiographical Soc. of Lond, 12 (1942), 94-108.

35. E. Witt, Theorie der quadratischen Formen in beliebigen Körpern, Journal fïr die Reine und Angewandete Mathematik, 176 (1937), 31-44.

36. — - Treue Darstellung Liescher Ringe, J. Reine Angew. Math., 177 (1937) 152-160.

37. M. Zorn, Theorie der Alternativen Ringe, Abh. Mat. Semi. Hamburg. Univ. 8 (1930), 123-147. 
